\author{
Universidade de São Paulo \\ Instituto de Física
}

\title{
Termodinâmica e eficiência de partículas brownianas em contato com reservatórios sequenciais
}

\section{Angel Luis Leiva Stable}

Orientador: Prof. Dr. Carlos Eduardo

Fiore dos Santos

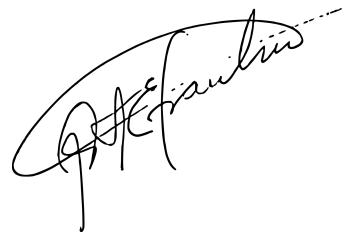

Tese de mestrado apresentada ao Instituto de Física como requisito parcial para a obtenção do título de Mestre em Ciências.

Banca Examinadora:

Prof. Dr. Carlos Eduardo Fiore dos Santos (Orientador)(IFUSP)

Prof. Dr. Eric de Castro e Andrade - IFSC USP

Prof. Dr. Marcus Vinicius Segantini Bonança - UNICAMP

São Paulo 
FICHA CATALOGRÁFICA

Preparada pelo Serviço de Biblioteca e Informação do Instituto de Física da Universidade de São Paulo

\section{Leiva Stable, Angel Luis}

Termodinâmica e eficiência de partículas brownianas em contato com reservatórios sequenciais. São Paulo, 2021.

Dissertação (Mestrado) - Universidade de São Paulo. Instituto de Física. Depto. de Física Geral

Orientador: Prof. Dr. Carlos Eduardo Fiore dos Santos

Área de Concentração: Física Estatística e Termodinâmica.

Unitermos: 1. Termodinâmica; 2. Rendimento; 3. Potência; 4. Partícula browniana.

USP/IF/SBI-051/2021 


\author{
University of São Paulo \\ Physics Institute
}

\title{
Thermodynamics and efficiency of Brownian particles in contact with sequential reservoirs
}

\section{Angel Luis Leiva Stable}

Supervirsor: Prof. Dr. Carlos Eduardo Fiore dos Santos

Thesis submitted to the Physics Institute of the University of São Paulo in partial fulfillment of the requirements for the degree of Master of Science.

Examining Committee:

Prof. Dr. Carlos Eduardo Fiore dos Santos (Supervisor)(IFUSP)

Prof. Dr. Eric de Castro e Andrade - IFSC USP

Prof. Dr. Marcus Vinicius Segantini Bonança - UNICAMP

São Paulo 
Dedicatória

Em especial à minha mãe. 


\section{Agradecimentos}

Agradeço neste trabalho ao meu orientador Dr. Carlos Eduardo Fiore do Santos pela contribuição, aos meus amigos, professores. O presente trabalho foi realizado com apoio do CNPq, Conselho Nacional de Desenvolvimento Científico e Tecnológico - Brasil. 


\section{Resumo}

Introduzimos uma abordagem denominada colisional para uma partícula browniana, na qual ela é colocada sequencialmente em contato com distintos reservatórios térmicos. Consideramos dois casos diferentes: mesmo intervalo de tempo a cada interação entre o sistema e o reservatório e o caso de intervalos de tempos diferentes. Em ambos casos, tal modelo pode ser usado como uma máquina térmica, apresentando solução exata para quaisquer temperaturas e forças externas. A produção de entropia e o trabalho apresentam um comportamento bilinear, onde obtemos os coeficientes de Onsager no regime de temperaturas iguais ou próximas entre os reservatórios. Uma analise detalhada acerca do papel de cada ingrediente (período, força e assimetria) bem como diferentes protocolos de optimização foram apresentados. Nossos resultados mostraram que a assimetria no período de interação constitui uma estrategia significativa para optimizar a máquina térmica, sobretudo em regimes onde a dependência diminui.

Palavras-chave: Termodinâmica, rendimento, potência, partícula browniana. 


\begin{abstract}
We introduce an approach called collisional for a Brownian particle, in which it is sequentially placed in contact with different thermal reservoirs. We consider two different cases: the same time interval at each interaction between the system and the reservoir and the case of different time intervals. In both cases, such a model can be used as a heat engine, presenting an exact solution for any external temperatures and forces. The entropy production and the work present a bilinear behavior, where we obtain the Onsager coefficients in the regime of equal or close temperatures between the reservoirs. A detailed analysis of the role of each ingredient (period, strength and asymmetry) as well as different optimization protocols were presented. Our results showed that asymmetry in the interaction period constitutes a significant strategy to optimize the heat engine, especially in regimes where dependence decreases.
\end{abstract}

Keywords: Thermodynamics, efficiency, power, brownian particle. 
Conteúdo

Dedicatória

Agradecimentos

Resumo

iii

Abstract

iv

1 Introdução 1

2 Aspectos gerais para Termodinâmica fora de equilíbrio. 6

2.1 Produção de entropia: Descrição macroscópica. . . . . . . . . . . . . . 6

2.2 Equação de Langevin. . . . . . . . . . . . . . . . . . . . . . 8

2.3 Equação de Fokker-Planck. . . . . . . . . . . . . . . . . . . . . . . . 10 
2.4 Produção de entropia: Descrição microscópica. . . . . . . . . . . . . . . 11

3 Partícula Browniana em contato com reservatórios térmicos sequenciais. 13

3.1 Descrição do modelo. . . . . . . . . . . . . . . . . . . . . . . 13

3.2 Solução exata para $N$ reservatórios térmicos sequenciais. . . . . . . . . . 14

3.3 Partículas brownianas em contato com dois reservatórios térmicos sobre a ação de forças externas. . . . . . . . . . . . . . . . . . . 18

3.3 .1 Força constante. . . . . . . . . . . . . . . 18

3.3.2 Força linear. . . . . . . . . . . . . . . 20

3.4 Forma bilinear e coeficientes de Onsager. . . . . . . . . . . . . . 21

3.5 Caso assimétrico. . . . . . . . . . . . . . . . 22

3.5.1 Caso assimétrico sem forças externas. . . . . . . . . . . . 22

3.5.2 Caso assimétrico com forças externas. . . . . . . . . . . . . . 24

4 Rendimento $\quad 28$

4.1 Introdução . . . . . . . . . . . . . . . . . . . . . . . . 28

4.2 Rendimento para temperaturas iguais. . . . . . . . . . . . . . 30

4.2 .1 Caso simétrico. . . . . . . . . . . . . . . 30

4.2 .2 Caso assimétrico. . . . . . . . . . . . . . . 32

4.3 Temperaturas diferentes. . . . . . . . . . . . . . . . 36

4.3 .1 Caso simétrico . . . . . . . . . . . . . . 36

4.3 .2 Caso assimétrico. . . . . . . . . . . . . . . . 38 
4.4 Descrição aproximada. . . . . . . . . . . . . . . . . . . . . . . . 40

5 Conclusões $\quad 46$

$\begin{array}{ll}\text { A Alguns cálculos analíticos. } & 48\end{array}$

A.1 Equação de Fokker-Planck para o movimento browniano. . . . . . . . . . 48

A.2 Solução da equação de Fokker-Planck. . . . . . . . . . . . . . . . . . . . 49

A.3 Produção de Entropia. . . . . . . . . . . . . . . . . . . . . . 51

A.4 Produção de entropia para o caso de força constante simétrico. . . . . . . . 54

A.5 Artigo publicado . . . . . . . . . . . . . . . 56 


\title{
CAPÍTULO 1
}

\author{
Introdução
}

A termodinâmica de equilíbrio, cujo objetivo reside no estudo das propriedades macroscópicas a partir das equações de estado, é baseada nas transformações reversíveis por meio de processos quase-estáticos e não leva em conta explicitamente o tempo. Porém em muitos sistemas (biológicos, reações químicas, máquinas térmicas) o sistema não está em equilíbrio termodinâmico, de forma que é desejável uma teoria capaz de explicar fenômenos que não estão em equilíbrio termodinâmico.

Nos últimos anos, a termodinâmica fora de equilíbrio, também conhecida como termodinâmica estocástica, tem avançado nesta direção, no sentido de generalizar para fenômenos fora de equilíbrio conceitos abordados no caso da termodinâmica de equilíbrio. Sob um ponto de vista microscópico, a condição de balanço detalhado $W(m, n) P(n)=$ $W(n, m) P(m)$ caracteriza se o sistema evoluirá para um estado de equilíbrio termodinâmico (cuja distribuição de probabilidades será a de Boltzmann-Gibbs e a ausência de correntes estacionárias não nulas), ou seja o sistema evoluirá para um estado de não equilíbrio termodinâmico (caracterizado por correntes estacionárias). 
O ponto de partida para a formulação de uma teoria estocástica na termodinâmica fora do equilíbrio requer duas definições: a de entropia e produção de entropia. No primeiro caso, estendemos a relação $S(t)=-k_{B} \sum_{m} P_{m}(t) \ln P_{m}(t)$ proposta no contexto de equilíbrio para sistemas fora do equilíbrio. O segundo ponto requer uma definição de taxa de produção de entropia. Neste caso, para sistemas markovianos, assumimos a definição proposta por Schnakenberg:

$$
\Pi=\frac{k_{B}}{2} \sum_{n m}[W(m, n) P(n, t)-W(n, m) P(m, t)] \ln \left[\frac{W(m, n) P(n, t)}{W(n, m) P(m, t)}\right] .
$$

Conforme mostraremos adiante, é possível encontrarmos uma relação análoga para sistemas descritos por equações de Fokker-Planck. Em ambos os casos, a produção de entropia é estritamente positiva para sistemas fora do equilíbrio (quando a condição de balanço detalhado é violada) e se anula para sistemas em equilíbrio. Cabe ressaltar que a conexão entre as definições acima com as quantidades macroscópicas é feita por meio da hipótese de balanço detalhado local [1].

Sob um ponto de vista genérico, a produção de entropia é dada pela Eq.[1.1] e pode ser escrita como uma soma de produtos dos chamados fluxos conjugados, $J_{i}$, e forças termodinâmicas, $X_{i}$, responsáveis pela manutenção do sistema fora de equilíbrio. Assim a generalização da segunda lei para um sistema físico qualquer pode ser escrita na forma:

$$
\bar{\Pi}=\sum_{i} J_{i} X_{i} \geqslant 0 \quad, \quad i=1,2,3, \ldots, n
$$

onde a igualdade ocorre no estado de equilíbrio termodinâmico (consistente com o balanço detalhado ser obedecido). Um regime importante é o linear (próximo do equilíbrio termodinâmico) em que as forças termodinâmicas podem ser tomadas como pequenas. Neste caso, os fluxos termodinâmicos podem ser tomados como uma combinação linear de todas as forças correspondentes:

$$
J_{i}=\sum_{j} L_{i j} X_{j}
$$

aqui os coeficientes $L_{i j}$ são os elementos da matriz de Onsager. No caso de forças termodinâmicas constantes, os coeficientes $L_{i j}$ satisfazem reciprocidade [2] ( $L_{i j}=L_{j i}, \forall i \neq$ $j$ ). Por outro lado, para forças dependentes do tempo eles estão satisfeitos pelas relações de Onsager-Casimir. 
Um dilema antigo na Termodinâmica diz respeito à questão de mitigar o impacto do ruído/desperdiço de energia sob a forma de calor a fim de melhorar o desempenho da máquina de uma forma realista. Isso constitui um problema de alta relevância, não apenas para fins teóricos [3], [4], [5], [6] , mas também para construção de aparatos experimentais [7], [8], [9], [10]. Tais questões têm sido extensivamente investigadas nos últimos anos tanto no escopo da Termodinâmica fora do equilíbrio quanto da Termodinâmica quântica. Várias abordagens têm sido empregadas não apenas para lidar com os objetivos acima mencionados, mas também para descrever vários fenômenos de fora do equilíbrio operando em nanoescala, como propriedades de transporte, sistemas biológicos, reações químicas, transições de fase, limites/restrições entre precisão e dissipação e outras.

Partículas brownianas têm sido bastante utilizadas para o estudo da eficiência fora do equilíbrio. Dentre os diferentes contextos e abordagens e estratégias usadas, algumas delas são implementadas explorando o desempenho por meio de forças temporais-periódicas distintas [6], [8], confinando e manipulando por meio de potenciais dependentes do espaço e do tempo [11]. Em muitos casos, uma vez que não é possível atingirmos a máxima eficiência de Carnot, tanto por imposições da segunda lei nem pelo fato de que tal regime requereria tempos suficientemente longos de relaxação para alcançarmos o regime de processos quase-estáticos, protocolos de optimização da potência têm sido amplamente considerados nos últimos anos. Um resultado importante neste contexto é a eficiência a máxima potência obtida por Curzon e Ahlborn [12], dada por $\eta_{C A}=1-\sqrt{T_{C} / T_{H}}$. Tal resultado notável também foi obtido em vários trabalhos [13], [14] a partir de outras ferramentas da termodinâmica estocástica [3], [7], e apesar de não possuir o mesmo status universal que a eficiência de Carnot, fornece um guia poderoso quanto à operação de motores fora de equilíbrio em situações mais realistas e esclarece a construção e o desempenho de motores de pequena escala (dispositivos nanoscópicos) trabalhando no regime de máxima potência. Isto porque mesmo que todas as fontes de dissipação fossem eliminadas, a obtenção de eficiências próximas da de Carnot requer a implementação de estados quase estáticos, de forma que a máquina operaria a potência nula, constituindo não apenas um regime ideal, como também impraticável.

Nesta dissertação de mestrado apresentamos a ideia de máquinas brownianas colisionais (ou modelos colisionais para partículas brownianas). Nosso propósito reside em otimizar o desempenho de máquinas irreversíveis operando em partes isotérmicas por 
meio do estudo de diferentes ingredientes, dentre eles o período, tempo de interação entre o sistema e o ambiente e a força externa. Tal abordagem tem sido empregada com sucesso em diferentes contextos, tais como sistemas que interagem com reservatórios distintos e/ou apenas com uma pequena fração do ambiente, sistema interagindo com o ambiente e apresentando distintos "drivings"sobre cada membro do sistema. Na primeira parte deste trabalho, consideramos tempos simétricos, isto é, cada interação entre a partícula e o reservatório ocorre a frações iguais do período [15]. Na segunda parte, avançamos no presente estudo e consideramos intervalos de tempo diferentes em cada interação. Isto porque, dependendo dos parâmetros (período e diferença de temperaturas), a versão simétrica pode ser operada de forma muito ineficiente. Nosso estudo consideramos maximizações para eficiência além da potência máxima e considerando diferentes protocolos de maximizações, incluindo a maximização da eficiência e não só respeito à força mas também à assimetria. Nossa descrição revela que ajustando adequadamente o tempo de interação, é possível aumentar substancialmente o desempenho da máquina sob diferentes perspectivas, incluindo não apenas sua eficiência e potência, mas também o regime da máquina aumenta substancialmente. Obtivemos expressões exatas para todas as quantidades termodinâmicas, dentre elas, calor, trabalho e produção de entropia. Uma atenção especial será dada ao caso de dois reservatórios pois poderá ser utilizado como uma máquina térmica operando a tempo finito. Consideramos o regime de temperaturas iguais e diferentes temperaturas entre os reservatórios,sendo que no primeiro caso uma relação entre as quantidades e os coeficientes de Onsager serão discutidas. Inicialmente introduzimos uma expressão aproximada para a eficiência (reportado na seção 4.4). Em seguida reformulamos o estudo da eficiência por meio da razão entre potência, trabalho e o calor trocado.

Esta dissertação está estruturada da seguinte forma: no segundo capítulo introduzimos os conceitos básicos da termodinâmica fora de equilíbrio bem como a abordagem teórica a ser usada nesta dissertação. No terceiro capítulo introduzimos o modelo colisional bem como as propriedades termodinâmicas, dentre elas, a produção de entropia (com ou sem forças externas) para caso simétrico e assimétrico. Expressões para os coeficientes de Onsager são obtidas em ambos casos. No quarto capítulo analisamos a eficiência e potência do modelo para temperaturas iguais e diferentes dos reservatórios térmicos. Finalmente, são apresentadas as conclusões sobre nosso trabalho no quinto capítulo. 
CAPÍTULO 1. INTRODUÇÃO 


\section{CAPÍTULO 2}

\section{Aspectos gerais para Termodinâmica fora de equilíbrio.}

\subsection{Produção de entropia: Descrição macroscópica.}

Considere dois sistemas físicos 1 e 2 isolados, onde $X_{i}^{1}, X_{i}^{2}$ representam versáveis extensivas de cada compartimento, como por exemplo: energia interna $(U)$, volume $(V)$, ou numero de partículas $(N)$, de forma que:

$$
X_{i}^{1}+X_{i}^{2}=A \quad, \quad d X_{i}^{1}=-d X_{i}^{2},
$$

onde $A$ é uma constante.

Uma vez que a entropia total no sistema $(S)$ é uma grandeza extensiva, temos que:

$$
S=S_{1}+S_{2},
$$

de forma que derivando a relação de acima na variável $X_{i}^{1}$ obtemos:

$$
\frac{\partial S}{\partial X_{i}^{1}}=\frac{\partial S_{1}}{\partial X_{i}^{1}}+\frac{\partial S_{2}}{\partial X_{i}^{1}}
$$


Por outro lado, da Eq.[2.1] temos que:

$$
\frac{\partial S}{\partial X_{i}^{1}}=\frac{\partial S_{1}}{\partial X_{i}^{1}}-\frac{\partial S_{2}}{\partial X_{i}^{2}}
$$

No equilíbrio termodinâmico, a variação da entropia total no sistema é máxima, portanto:

$$
\frac{\partial S_{1}}{\partial X_{i}^{1}}=\frac{\partial S_{2}}{\partial X_{i}^{2}}
$$

o que é equivalente entre a igualdade das temperaturas, pressões e potenciais químicos.

Tomando a derivada de $S$ com relação ao tempo, temos:

$$
\frac{d S}{d t}=\sum_{i}\left[\frac{\partial S_{1}}{\partial X_{i}^{1}} \frac{d X_{i}^{1}}{d t}+\frac{\partial S_{2}}{\partial X_{i}^{2}} \frac{d X_{i}^{2}}{d t}\right]
$$

e usando a Eq.[2.1] chegamos em:

$$
\frac{d S}{d t}=\sum_{i}\left[\frac{\partial S_{2}}{\partial X_{i}^{2}} \frac{d X_{i}^{2}}{d t}-\frac{\partial S_{1}}{\partial X_{i}^{1}} \frac{d X_{i}^{2}}{d t}\right]
$$

Chamando o termo $d X_{i}^{2} / d t$ como corrente microscópica $J_{i}$, ou como fluxo termodinâmico, a relação Eq.[2.7] pode ser reescrita da seguente forma:

$$
\frac{d S}{d t}=\sum_{i}\left[\frac{\partial S_{2}}{\partial X_{i}^{2}}-\frac{\partial S_{1}}{\partial X_{i}^{1}}\right] J_{i},
$$

onde a diferença:

$$
\frac{\partial S_{2}}{\partial X_{i}^{2}}-\frac{\partial S_{1}}{\partial X_{i}^{1}}=F_{i}
$$

identifica-se como a "força generalizada" $F_{i}$.

Dessa forma, a quantidade $d S / d t$, denominada taxa de produção de entropia, pode ser escrita como uma soma de produtos de fluxos e forças:

$$
\frac{d S}{d t}=\sum_{i} J_{i} F_{i}
$$

Na expressão de acima, definimos $d S / d t$ como a produção de entropia devido a troca irreversível de calor/substancia entre os subsistemas 1 e 2. Um caso importante é aquele em que $V_{2} \gg V_{1}$ ( também $N_{2} \gg N_{1}$ e $U_{2} \gg U_{1}$ ), de forma que o subsistema 2 atua como um reservatório térmico de 1 . Neste caso, a produção de entropia deve-se ao subsistema 1. 


\begin{tabular}{|c|c|c|}
\hline Fluxo de entropia & Variação temporal da entropia & Produção de entropia \\
\hline$\sum_{i} J_{i} \frac{\partial S_{2}}{\partial X_{i}^{2}}=\Phi$ & $-\sum_{i} J_{i} \frac{\partial S_{1}}{\partial X_{i}^{1}}=\frac{d S_{1}}{d t}$ & $\frac{d S}{d t}=\Pi$ \\
\hline
\end{tabular}

Reescrevendo a Eq.[2.8] neste caso, temos que:

$$
\frac{d S_{1}}{d t}=\Pi-\Phi
$$

No estado estacionário $d S / d t=0$, ou seja: $\Phi=\Pi$, de forma que para sistemas em equilíbrio $\Phi=\Pi=0$, enquanto para sistemas fora de equilíbrio $\Phi=\Pi>0$.

Como último comentário, ressaltamos que a expressão Eq.[2.11] será obtida no próximo capítulo por meio da equação de Fokker-Planck.

\subsection{Equação de Langevin.}

A segunda lei de Newton estabelece como uma força arbitrária provoca uma mudança na velocidade de um sistema físico qualquer. No regime microscópico, as flutuações na posição e velocidade precisam ser levadas em conta. Neste caso, podemos tratar de forma mais simples $x(t)$ e $v(t)$ descrevendo a dinâmica da partícula browniana em um meio viscoso sob a ação de colisões aleatórias independentes e um termo de ruido $\xi_{i}(t)$ que manifesta o caráter estocástico do sistema. Neste caso, temos então que:

$$
\frac{d v_{i}}{d t}+f(v)=\xi_{i}(t)
$$

que é a equação de Langevin.

A ideia fundamental de Langevin foi tratar a viscosidade e as flutuações da velocidade como efeitos complementares de um único fenômeno de subescala: colisões entre as moléculas do fluido e a partícula browniana. Neste caso as colisões contribuem ao termo de fluatações $\xi_{i}(t)$ entre a partícula e o meio, onde o ruido $\xi_{i}(t)$ satisfaz as seguintes propriedades:

$$
\left\langle\xi_{i}(t)\right\rangle=0 \quad, \quad\left\langle\xi_{i}(t) \xi_{i^{\prime}}\left(t^{\prime}\right)\right\rangle=\Gamma_{i} \delta\left(t-t^{\prime}\right)
$$

A "força de Langevin"em Eq.[2.13] é referida na literatura como "ruido branco". A partir das relações Eqs.[2.12-2.13]. A equação diferencial Eq.[2.12] em geral é governada 
por um processo de Ornstein-Uhlenbeck [16], o mesmo com caráter estocástico descreve a evolução temporal de uma partícula browniana em um meio viscoso, leva o nome por Leonard Ornstein (1880-1941) e George Eugene Uhlenbeck (1900-1988).

O movimento aleatório de partículas imersas em uma solução foi originalmente observado em 1828 pelo botânico inglês Robert Brown (1773-1858), onde ele notou que as partículas em suspensão adquiriam uma espécie de movimento errático, o mesmo tempo depois, ficaria popularmente conhecido pelo nome de movimento browniano (BM) [16]. Supondo uma solução de Eq.[2.12] para uma força $f(v)=-\gamma v$ da seguinte forma:

$$
v(t)=u(t) e^{-\gamma t}
$$

onde $\gamma$ está relacionado com a viscosidade do sistema. Substituindo Eq.[2.12] a solução de acima, temos que:

$$
\frac{d u}{d t}=e^{\gamma t} \xi(t)
$$

assim a solução de Eq.[2.15] tem a forma:

$$
u=u_{0}+\int_{0}^{t} e^{\gamma t^{\prime}} \xi\left(t^{\prime}\right) d t^{\prime}
$$

e então temos que:

$$
v=v_{0} e^{-\gamma t}+e^{-\gamma t} \int_{0}^{t} e^{\gamma t^{\prime}} \xi\left(t^{\prime}\right) d t^{\prime}
$$

Usando a primeira das propriedades Eq.[2.13], chegamos em:

$$
\langle v\rangle=v_{0} e^{-\gamma t}
$$

Assim subtraindo as relações Eqs.[2.17-2.18] temos que:

$$
v-\langle v\rangle=e^{-\gamma t} \int_{0}^{t} e^{\gamma t^{\prime}} \xi\left(t^{\prime}\right) d t^{\prime} .
$$

Para o cálculo da variância, devemos elevar Eq.[2.19] ao quadrado, de forma que:

$$
(v-\langle v\rangle)^{2}=e^{-2 \gamma t} \int_{0}^{t} \int_{0}^{t} e^{\gamma\left(t^{\prime}+t^{\prime \prime}\right)} \xi\left(t^{\prime}\right) \xi\left(t^{\prime \prime}\right) d t^{\prime} d t^{\prime \prime}
$$

tomando a equação anterior e usando a segunda das propriedades Eq.[2.13] temos:

$$
\left\langle(v-\langle v\rangle)^{2}\right\rangle=e^{-2 \gamma t} \int_{0}^{t} \Gamma e^{-2 \gamma t^{\prime}} d t^{\prime}
$$


de onde chegamos a seguente expressão:

$$
\left\langle v^{2}\right\rangle=\frac{\Gamma}{2 \gamma}\left(1-e^{-2 \gamma t}\right)+v_{0}^{2} e^{-2 \gamma t} .
$$

Portanto no estado estacionário, podemos obter o valor médio da energia da partícula browniana:

$$
\langle E\rangle=\frac{1}{2} m\left\langle v^{2}\right\rangle=\frac{m \Gamma_{i}}{4 \gamma},
$$

onde a constante $\Gamma_{i}$ está relacionada com a temperatura do reservatório $i$-ésimo, por meio do teorema de equipartição da energia $\langle E\rangle=\frac{1}{2} k_{B} T$, de forma que:

$$
\Gamma_{i}=\frac{2 \gamma k_{B} T_{i}}{m}
$$

\subsection{Equação de Fokker-Planck.}

Para o movimento browniano a evolução temporal da distribuição de probabilidades está descrita pela equação de Fokker-Planck, cuja demostração será mostrada no apêndice (A.1):

$$
\frac{\partial}{\partial t} P(v, t)=-\frac{\partial}{\partial v}[f(v) P(v, t)]+\frac{\Gamma}{2} \frac{\partial^{2}}{\partial v^{2}} P(v, t)
$$

A relação Eq.[2.25] permite encontrar a função de distribuição das partículas brownianas $\rho(v, t)$ se as condições iniciais e de contorno forem bem definidas.

Partindo da equação de Langevin em uma variável:

$$
\frac{d v}{d t}=f(v)+\xi(t)
$$

onde para $f(v)=c$, as relações Eqs.[2.25-2.26] ficam neste caso:

$$
\frac{\partial P}{\partial t}=-c \frac{\partial P}{\partial v}+\frac{\Gamma}{2} \frac{\partial^{2} P}{\partial^{2} t} \quad, \quad \frac{d v}{d t}=c+\xi(t)
$$

no caso $f(v)=-\gamma v$ a distribuição de probabilidades está dada por:

$$
P(v, t)=\frac{1}{\sqrt{2 \pi b(t)}} e^{-\frac{[v-a(t)]^{2}}{2 b(t)}}
$$

onde as quantidades médias $a(t)$ e $b(t)$ ficam :

$$
a(t)=v_{0} e^{-\gamma t} \quad, \quad b(t)=\frac{\Gamma}{2 \gamma}\left(1-e^{-\gamma t}\right),
$$


respetivamente.

No caso da distribuição estacionária $(t \rightarrow \infty)$, temos $b(t) \rightarrow \Gamma / 2 \gamma$, e então:

$$
P(v)=\sqrt{\frac{\gamma}{\pi \Gamma}} e^{-\frac{\gamma v^{2}}{\Gamma}} .
$$

Substituindo Eq.[2.23] em Eq.[2.31], chegamos em:

$$
P(v)=\sqrt{\frac{m}{2 \pi k_{B} T}} e^{-\frac{m v^{2}}{2 k_{B} T}},
$$

consistente com a distribuição Maxwell-Boltzmann-Gibbs no equilíbrio termodinâmico. No caso de um estado estacionário de não equilíbrio, $P(v, t)$ tem a forma Eq.[2.28], porém $a(t)$ e $b(t)$ serão diferentes das expressões acima.

\subsection{Produção de entropia: Descrição microscópica.}

O ponto de partida para a construção de uma teoria termodinâmica fora de equilíbrio consiste em assumir que expressão para a entropia de um sistema físico pode ser escrita pela entropia de Shannon:

$$
S(t)=-\int P(v, t) \ln [P(v, t)] d v
$$

A partir da equação de Fokker-Planck:

$$
\frac{\partial P(v, t)}{\partial t}=-\frac{\partial J}{\partial v}
$$

que foi reescrita sobre a forma de equação de continuidade:

$$
J=f_{i} P(v, t)-\frac{\Gamma_{i}}{2} \frac{\partial P(v, t)}{\partial v},
$$

onde $J$ é a corrente de probabilidade, que se anula no equilíbrio termodinâmico, chegamos para Eq.[2.32] em:

$$
\frac{d S}{d t}=\int \ln [P(v, t)] \frac{\partial J}{\partial v} d v
$$

efetuando uma integração por partes em Eq.[2.35] e considerando que $P(v, t)$ e $J$ são nulas nas extremidades, chegamos em:

$$
\frac{d S}{d t}=\int J \frac{\partial}{\partial v} \ln [P(v, t)] d v
$$


Usando novamente Eq.[2.34], podemos reescrever o logaritmo da distribuição de probabilidades da seguinte forma:

$$
\frac{\partial}{\partial v} \ln [P]=\frac{2 f_{i}}{\Gamma_{i}}-\frac{2 J}{\Gamma_{i} P}
$$

Por substituição direta de Eq.[2.37] em Eq.[2.36] a varição temporal da entropia fica:

$$
\frac{d S}{d t}=-\frac{2}{\Gamma_{i}} \int f_{i} J d v+\frac{2}{\Gamma_{i}} \int \frac{J^{2}}{P} d v
$$

onde o segundo termo em Eq.[2.38] identifica-se com a produção de entropia e é dado por:

$$
\Pi=\frac{2}{\Gamma_{i}} \int \frac{J^{2}}{P} d v
$$

e o primeiro termo corresponde ao fluxo de entropia:

$$
\Phi=\frac{2}{\Gamma_{i}} \int f_{i} J d v
$$

No estado estacionário de equilíbrio onde $J=0$, temos $\Pi=\Phi=0$. Por outro lado, no estado estacionário de não equilíbrio temos que $J \neq 0$ e $\Pi=\Phi>0$.

Note que, a equação acima ainda pode ser reescrita como:

$$
\frac{d S_{i}}{d t}=\Pi_{i}(t)-\Phi_{i}(t)
$$

onde o fluxo de entropia acima pode ser reescrito como na forma:

$$
\Phi_{i}(t)=\frac{\dot{Q}_{i}}{T_{i}}=\gamma_{i}\left(\frac{m}{T_{i}}\left\langle v_{i}^{2}\right\rangle-k_{B}\right) .
$$

Na equação anterior o fluxo está relacionado com $\left\langle v_{i}^{2}\right\rangle$, de forma que podemos calcular a produção de entropia efetuando a integração de Eq.[2.42] em um período.

Novamente, no caso do regime estacionário de equilíbrio, temos que $\left\langle v_{i}^{2}\right\rangle=k_{B} T_{i} / 2$, o que é consistente com $\Pi=\Phi=0$ no equilíbrio. 


\section{CAPÍTULO 3}

\section{Partícula Browniana em contato com reservatórios térmicos sequenciais.}

\subsection{Descrição do modelo.}

Considerando agora uma versão generalizada, devido a presença de forças externas, no qual a partícula browniana está em contato com $N$-reservatórios térmicos sequenciais cada um a uma temperatura arbitrária [15], de modo que a partícula esteja em contato com cada um deles durante um intervalo de tempo $\tau / N$ a uma temperatura $T_{i}$. A dinâmica novamente é descrita pela equação de Langevin, mencionada anteriormente e dada abaixo:

$$
\frac{d v_{i}}{d t}=-\gamma_{i} v_{i}+f_{i}(t)+\xi_{i}(t)
$$

onde: $f_{i}(t)=F_{i}(t) / m$ corresponde com a força externa e o subíndice $i$ está relacionado com o reservatório térmico $i$-ésimo.

Da mesma forma que fizemos no capitulo anterior, os termos associados com $\gamma_{i}$ estão relacionados com a viscosidade do sistema (interação da partícula com as moléculas 
do fluido) e $\xi_{i}(t)$ é o ruido estocástico que representa o efeito das colisões da partícula também com o fluido. Novamente, trata-se de um ruido branco, descrito por:

$$
\left\langle\xi_{i}(t)\right\rangle=0 \quad, \quad\left\langle\xi_{i}(t) \xi_{i}^{\prime}\left(t^{\prime}\right)\right\rangle=2 \gamma_{i} T_{i} \delta_{i i^{\prime}} \delta\left(t-t^{\prime}\right)
$$

sendo $T_{i}$ a temperatura do reservatório $i$.

Neste caso, a equação de Fokker-Planck (FPE) novamente dada por:

$$
\frac{\partial P_{i}}{\partial t}=-\frac{\partial J_{i}}{\partial v}-f_{i}(t) \frac{\partial P_{i}}{\partial v},
$$

onde $J_{i}$ tem a forma de Eq.[2.34]. As expressões para a produção de entropia e fluxo de entropia são as mesmas que aquelas descritas no capítulo anterior e portanto serão omitidas. Por outro lado, a energia média, definida por:

$$
U_{i}=\left\langle E_{i}\right\rangle=\left\langle\frac{m v_{i}^{2}}{2}\right\rangle
$$

tem evolução temporal dada por:

$$
\frac{d U_{i}}{d t}=-\frac{m}{2} \int v^{2}\left[\frac{\partial J_{i}}{\partial v}+f_{i}(t) \frac{\partial P_{i}}{\partial v}\right] d v .
$$

A relação acima mostra que o comportamento energético do sistema é a soma de dois termos, um relacionado ao trabalho por unidade de tempo feito pela força sobre a partícula e o outro é o calor trocado no sistema, este ultimo também por unidade de tempo:

$$
\frac{d U_{i}}{d t}=\frac{d W}{d t}+\frac{d Q}{d t}
$$

de forma que Eq.[3.6] tem a forma da primeira lei da Termodinâmica. Reescrevendo o segundo termo de Eq.[3.5] assumindo que a distribuição de probabilidades tanto como sua derivada são nulas nas extremidades, temos que:

$$
\frac{d W_{i}}{d t}=-m\left\langle v_{i}\right\rangle(t) f_{i} \quad, \quad \frac{d Q_{i}}{d t}=-m\left(\gamma\left\langle v_{i}^{2}\right\rangle(t)-\frac{\Gamma_{i}}{2}\right) .
$$

\subsection{Solução exata para $N$ reservatórios térmicos sequen- ciais.}

Apresentadas as principais relações para as propriedades termodinâmicas, estamos em posição para obter seus valores no caso de reservatórios térmicos sequenciais. Uma vez 
que elas dependem de $\left\langle v_{i}\right\rangle(t)$ e $\left\langle v_{i}^{2}\right\rangle(t)$, suas expressões são dadas por:

$$
\frac{d}{d t}\left\langle v_{i}\right\rangle=-\gamma_{i}\left\langle v_{i}\right\rangle+f_{i}(t) \quad, \quad \frac{d}{d t} b_{i}(t)=-2 \gamma_{i} b_{i}(t)+\Gamma_{i},
$$

onde $b_{i}(t)=\left\langle v_{i}^{2}\right\rangle(t)-\left\langle v_{i}\right\rangle^{2}(t)$ é a variância da velocidade e $\Gamma_{i}=2 \gamma_{i} k_{B} T_{i} / m$.

Conforme mencionamos anteriormente, a forma da distribuição de probabilidades é gaussiana e tanto ela como as derivadas são nulas nas extremidades, além de isso é dependente da velocidade e do tempo na forma:

$$
P_{i}(v, t)=\frac{e^{-\left(v-\left\langle v_{i}\right\rangle\right)^{2} / 2 b_{i}(t)}}{\sqrt{2 \pi b_{i}(t)}},
$$

A partir das Eqs.[3.8] vemos que as expressões para $\left\langle v_{i}\right\rangle(t)$ e $b_{i}(t)$ adquirem a forma:

$$
\left\langle v_{i}\right\rangle(t)=e^{-\gamma_{i}\left(t-\tau_{i-1}\right)}\left[v_{i-1}^{\prime}+\int_{\tau_{i-1}}^{t} e^{\gamma_{i}\left(t^{\prime}-\tau_{i-1}\right)} f_{i}\left(t^{\prime}\right) d t^{\prime}\right],
$$

$\mathrm{e}$

$$
b_{i}(t)=A_{i-1} e^{-2 \gamma_{i}\left(t-\tau_{i-1}\right)}+\frac{\Gamma_{i}}{2 \gamma_{i}}
$$

As quantidades $v_{i}^{\prime}$ são calculadas a partir das condições de contorno:

$$
\left\langle v_{1}\right\rangle(0)=\left\langle v_{N}\right\rangle(\tau) \quad, \quad\left\langle v_{i}\right\rangle\left(\tau_{i}\right)=\left\langle v_{i+1}\right\rangle\left(\tau_{i}\right)
$$

Procedendo de forma análoga pela variância temos:

$$
b_{1}(0)=b_{N}(\tau) \quad, \quad b_{i}\left(\tau_{i}\right)=b_{i+1}\left(\tau_{i}\right) \quad ; \quad\{i=1 ; \ldots ; N\} .
$$

As expressões acima são exatas e valem para qualquer numero de reservatórios e forças eternas. Começando com um caso genérico de $N$ reservatórios e ausência de forças externas, temos que a Eq.[3.11] pode ser reescrita como:

$$
A_{i+1}=x A_{i}+\frac{1}{2 \gamma}\left(\Gamma_{i}-\Gamma_{i+1}\right),
$$

onde: $x=e^{-2 \gamma \tau / N}$, de forma que os coeficientes $A_{i}$ podem ser calculados pela relação de recorrência:

$$
A_{i}=x^{i-1} A_{1}+\frac{1}{2 \gamma} \sum_{l=2}^{i} x^{i-l}\left(\Gamma_{l-1}-\Gamma_{l}\right) \quad, \quad\{i=2 ; \ldots ; N\}
$$


Resolvendo Eq.[3.10] podemos encontrar todos os coeficientes $A_{i}^{\prime} s$, dados por:

$$
A_{1}=\frac{1}{2 \gamma} \frac{x^{N}}{1-x^{N}} \sum_{l=1}^{N} x^{-l}\left(\Gamma_{l}-\Gamma_{l+1}\right)
$$

$\mathrm{e}$

$$
A_{i}=\frac{1}{2 \gamma} \frac{x^{i-1}}{1-x^{N}} \times\left[\sum_{l=1}^{i-1} x^{-l}\left(\Gamma_{l}-\Gamma_{l+1}\right)+\sum_{l=i}^{N} x^{N-l}\left(\Gamma_{l}-\Gamma_{l+1}\right)\right],
$$

respectivamente. Note que $A_{i}^{\prime} s$ somente dependem de $\gamma, \tau, N$ e da diferencia de temperatura.

A partir de Eq.[3.17], assumindo que o sistema está em um estado estacionário de não equilíbrio podemos encontrar a produção de entropia sobre um período $(\tau)$ dada por:

$$
\bar{\Pi}=\frac{1}{\tau} \sum_{i=1}^{N} \int_{\tau_{i-1}}^{\tau_{i}} \Phi_{i}(t) d t=\frac{\left(1-e^{-2 \gamma \tau / N}\right)}{2 \gamma \tau} \sum_{i=1}^{N} \frac{A_{i}}{\Gamma_{i}},
$$

onde também combinando Eqs.[3.16-3.17] pode-se mostrar que:

$$
\sum_{i=1}^{N} \frac{A_{i}}{\Gamma_{i}}=\frac{x^{N}}{1-x^{N}} \sum_{i, l=1}^{N} x^{-l}\left(\frac{\Gamma_{i+l-1}-\Gamma_{i+l}}{\Gamma_{i}}\right) .
$$

Substituindo a expressão de acima em Eq.[3.18], chegamos na expressão:

$$
\bar{\Pi}=-\frac{N}{2 \gamma \tau}\left(\frac{1-x}{x}\right)+\frac{1}{2 \gamma \tau} \cdot \frac{x^{N-1}(1-x)^{2}}{1-x^{N}} \sum_{i, l=1}^{N} x^{-l} \frac{\Gamma_{i+l}}{\Gamma_{i}} .
$$

Para mostrarmos que $\Pi \geq 0$ usamos a desigualdade:

$$
\sum_{i=1}^{N} \frac{\Gamma_{i+l}}{\Gamma_{i}} \geq N \sqrt[N]{\prod_{i=1}^{N} \frac{\Gamma_{i+l}}{\Gamma_{i}}} \Longleftrightarrow \sum_{i=1}^{N} \frac{\Gamma_{i+l}}{\Gamma_{i}} \geq N
$$

e substituindo Eq.[3.21] em Eq.[3.20] é possível demostrar que:

$$
\bar{\Pi} \geq-\frac{N}{2 \gamma \tau}\left(\frac{1-x}{x}\right)+\frac{N}{2 \gamma \tau}\left(\frac{1-x}{x}\right)=0,
$$

consistente com a segunda lei da termodinâmica para sistemas fora de equilíbrio, quaisquer que sejam as temperaturas dos reservatórios.

A fim de ilustrarmos melhor os resultados de acima, vamos considerar o caso de dois reservatórios, em que $A_{1}$ e $A_{2}$ são dados por:

$$
A_{1}=\frac{\Gamma_{2}-\Gamma_{1}}{2 \gamma}\left(\frac{1-e^{-\gamma \tau}}{1-e^{-2 \gamma \tau}}\right)=\frac{\Gamma_{2}-\Gamma_{1}}{2 \gamma}\left(\frac{1}{1+e^{\gamma \tau}}\right),
$$


onde: $A_{1}=-A_{2}$, de forma que os fluxos de entropia ficam:

$$
\Phi_{1}(t)=\gamma\left(\frac{\Gamma_{2}-\Gamma_{1}}{\Gamma_{1}}\right)\left(\frac{1}{1+e^{2 \gamma \tau}}\right) e^{-2 \gamma t}, \quad 0 \leq t<\tau / 2
$$

$\mathrm{e}$

$$
\Phi_{2}(t)=\gamma\left(\frac{\Gamma_{1}-\Gamma_{2}}{\Gamma_{2}}\right)\left(\frac{1}{1+e^{2 \gamma \tau}}\right) e^{-2 \gamma\left(t-\frac{\tau}{2}\right)}, \quad \tau / 2 \leq t<\tau,
$$

respectivamente.

Uma vez calculados os fluxos Eqs.[3.24-3.25] e integrando em um período temos a produção de entropia total do sistema:

$$
\Pi=\bar{\Phi}=\frac{1}{\tau}\left[\int_{0}^{\tau / 2} \Phi_{1}(t) d t+\int_{\tau / 2}^{\tau} \Phi_{2}(t) d t\right],
$$

portanto substituindo Eqs.[3.24-3.25] em Eq.[3.26] chegamos em:

$$
\bar{\Pi}=\frac{\Gamma_{1} \Gamma_{2}}{2 \tau} \tanh \left(\frac{\gamma \tau}{2}\right)\left(\frac{1}{\Gamma_{1}}-\frac{1}{\Gamma_{2}}\right)^{2}
$$

É importante olhar que quando $\left(\Gamma_{1}=\Gamma_{2}\right)$ a produção de entropia é nula, consistente com o fato da diferença de temperaturas ser a única grandeza física encarregada de manter o sistema fora de equilíbrio neste caso.

Em particular, nos limites de baixos e altos períodos (em comparação com $1 / \gamma$ ), chegamos em:

$$
\bar{\Pi} \approx \frac{\Gamma_{1} \Gamma_{2}}{2 \tau}\left(\frac{1}{\Gamma_{1}}-\frac{1}{\Gamma_{2}}\right)^{2} \quad, \quad \bar{\Pi}=\frac{\Gamma_{1} \Gamma_{2} \gamma}{4}\left(\frac{1}{\Gamma_{1}}-\frac{1}{\Gamma_{2}}\right)^{2}
$$

respectivamente, ou seja o fato que a produção de entropia anula-se para $\tau \rightarrow \infty$, indicando que o sistema atinge ao equilíbrio termodinâmico nesse limite, no caso de tempos muito pequenos $(\tau \ll 1)$ temos que $\bar{\Pi}$ é máxima . Rescrevendo a produção de entropia da forma $\bar{\Pi}=\mathcal{J}_{T} f_{T}$, onde: $f_{T}=\left(1 / \Gamma_{2}-1 / \Gamma_{1}\right)$, dependente de uma força termodinâmica que está relacionada com a diferença de temperaturas entre os reservatórios, e o fluxo da mesma $\mathcal{J}_{T}=L_{T T} f_{T}$, onde: $L_{T T}$ é o coeficiente de Onsager relacionado com a temperatura, chegamos em:

$$
L_{T T}=\frac{\Gamma_{1} \Gamma_{2}}{2 \tau} \tanh \left(\frac{\gamma \tau}{2}\right)
$$

pois $L_{T T} \geq 0$, já que $\left(\Gamma_{1}, \Gamma_{2}, \tau, \gamma\right)$ de Eq.[3.29] são sempre positivos, e então: $(\gamma \tau / 2) \geq$ $0 \Rightarrow \tanh (\gamma \tau / 2) \geq 0$. 


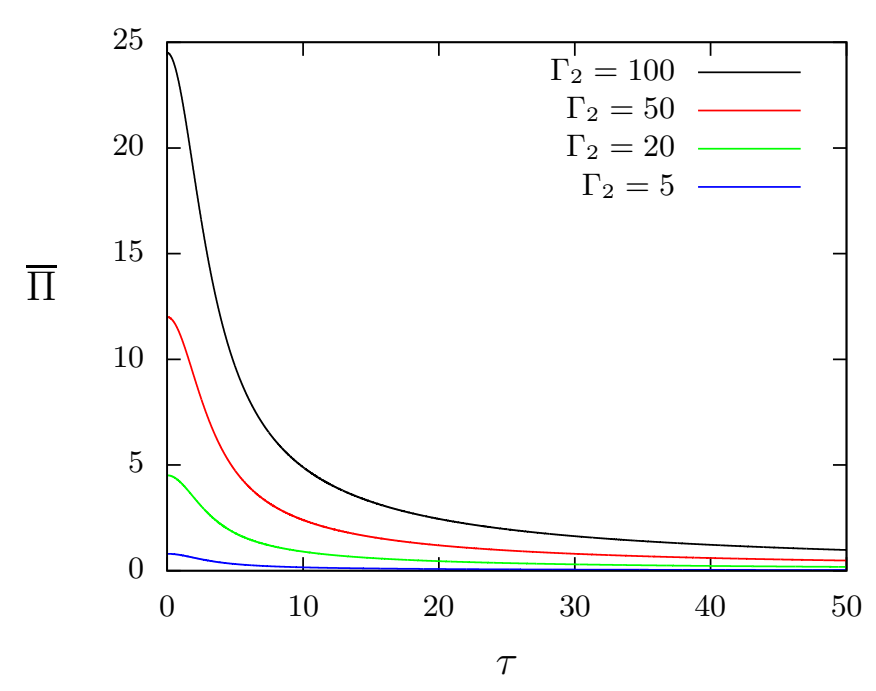

Figura 3.1: Produção de entropia Eq.[3.27] versus $(\tau)$ para $\left(\gamma=1, \Gamma_{1}=2\right)$ e diferentes valores de $(\Delta \Gamma$ 's).

\subsection{Partículas brownianas em contato com dois reserva- tórios térmicos sobre a ação de forças externas.}

Para o nosso propósito, que consiste em estudar a optimização de máquinas brownianas a tempos finitos, vamos considerar inicialmente o caso de partículas brownianas operando a tempos simétricos, de forma que em cada metade a partícula está sujeita a uma força externa diferente. Consideremos aqui dois protocolos para forças dados por:

$$
f_{i}(t)= \begin{cases}f_{1} ; & 0 \leq t<\tau / 2 \\ f_{2} ; & \tau / 2 \leq t<\tau\end{cases}
$$

$\mathrm{e}$

$$
\frac{f_{i}(t)}{\gamma}=\left\{\begin{array}{c}
\lambda_{1} t ; \quad 0 \leq t<\tau / 2 \\
\lambda_{2}\left(\frac{\tau}{2}-t\right) ; \quad \tau / 2 \leq t<\tau
\end{array},\right.
$$

de forma que temos no primeiro caso uma força constante e no segundo uma que varia linearmente no tempo.

\subsubsection{Força constante.}

No caso de força constante, as expressões para $\left\langle v_{1}\right\rangle(t)$ e $\left\langle v_{2}\right\rangle(t)$ estão dadas por: 


$$
\langle v\rangle=\left\{\begin{array}{c}
\left\langle v_{1}\right\rangle(t)=\frac{e^{\gamma \tau / 2}}{\gamma}\left(\frac{f_{2}-f_{1}}{1+e^{\gamma \tau / 2}}\right) e^{-\gamma t}+\frac{f_{1}}{\gamma} \\
\left\langle v_{2}\right\rangle(t)=\frac{e^{\gamma \tau / 2}}{\gamma}\left(\frac{f_{1}-f_{2}}{1+e^{\gamma \tau / 2}}\right) e^{-\gamma(t-\tau / 2)}+\frac{f_{2}}{\gamma}
\end{array}\right.
$$

respectivamente.

Substituindo Eq.[3.32] em Eq.[3.7] temos que o trabalho em cada etapa é dado por:

$$
\overline{\dot{W}}_{1}=-\frac{m f_{1}}{\tau} \int_{0}^{\tau / 2}\left\langle v_{1}\right\rangle d t=\frac{m f_{1}}{\gamma^{2} \tau}\left(f_{1}-f_{2}\right) \tanh \left(\frac{\gamma \tau}{4}\right)-\frac{m f_{1}^{2}}{2 \gamma},
$$

$\mathrm{e}$

$$
\overline{\dot{W}}_{2}=-\frac{m f_{2}}{\tau} \int_{\tau / 2}^{\tau}\left\langle v_{2}\right\rangle d t=\frac{m f_{2}}{\gamma^{2} \tau}\left(f_{2}-f_{1}\right) \tanh \left(\frac{\gamma \tau}{4}\right)-\frac{m f_{2}^{2}}{2 \gamma}
$$

De forma análoga os calores médios $\bar{Q}_{i}^{\prime} s$ são dados por:

$$
\begin{aligned}
\overline{\dot{Q}}_{1} & =\frac{m}{4 \gamma \tau}\left(\Gamma_{2}-\Gamma_{1}\right) \tanh \left(\frac{\gamma \tau}{2}\right)+\frac{m}{2 \gamma^{2} \tau}\left(f_{1}+f_{2}\right)^{2} \times \\
& \times \tanh \left(\frac{\gamma \tau}{4}\right)+\frac{2 m f_{1}^{2}}{\gamma^{2} \tau}\left[\frac{\gamma \tau}{4}-\tanh \left(\frac{\gamma \tau}{4}\right)\right],
\end{aligned}
$$

$\mathrm{e}$

$$
\begin{aligned}
\overline{\dot{Q}}_{2} & =\frac{m}{4 \gamma \tau}\left(\Gamma_{1}-\Gamma_{2}\right) \tanh \left(\frac{\gamma \tau}{2}\right)+\frac{m}{2 \gamma^{2} \tau}\left(f_{2}+f_{1}\right)^{2} \times \\
& \times \tanh \left(\frac{\gamma \tau}{4}\right)+\frac{2 m f_{2}^{2}}{\gamma^{2} \tau}\left[\frac{\gamma \tau}{4}-\tanh \left(\frac{\gamma \tau}{4}\right)\right],
\end{aligned}
$$

respectivamente.

Note que tanto $\overline{\dot{W}}_{2}$ quanto $\overline{\dot{Q}}_{2}$ correspondem a $\bar{W}_{1}$ e $\overline{\dot{Q}}_{1}$ quando trocamos a ordem dos reservatórios $1 \leftrightarrow 2$ (e vice-versa). È importante notar que $\overline{\dot{Q}}_{1}+\overline{\dot{Q}}_{2}+\overline{\dot{W}}_{1}+\overline{\dot{W}}_{2}=0$, em consistência com a primeira lei da termodinâmica já que em um ciclo a variação da energia interna é nula (função de estado). A produção de entropia dada pela Eq.[2.42] pode ser reescrita da seguente forma:

$$
\bar{\Pi}=\frac{2 \gamma}{m}\left(\frac{\bar{Q}_{1}}{\Gamma_{1}}+\frac{\bar{Q}_{2}}{\Gamma_{2}}\right)
$$

e substituindo as expressões para $\left\langle v_{1}^{2}\right\rangle(t)=\left\langle v_{1}\right\rangle^{2}(t)+b_{1}(t)$ e $\left\langle v_{2}^{2}\right\rangle(t)=\left\langle v_{2}\right\rangle^{2}(t)+b_{2}(t)$ 
e calculando seus valores médios sobre um período chegamos na seguinte expressão:

$$
\begin{aligned}
\bar{\Pi} & =\frac{1}{2 \tau} \frac{\left(\Gamma_{2}-\Gamma_{1}\right)^{2}}{\Gamma_{1} \Gamma_{2}} \tanh \left(\frac{\gamma \tau}{2}\right)+\frac{1}{\gamma \tau}\left(\frac{1}{\Gamma_{1}}+\frac{1}{\Gamma_{2}}\right) \times \\
& \times \tanh \left(\frac{\gamma \tau}{4}\right)\left(f_{1}+f_{2}\right)^{2}+\left(\frac{f_{1}^{2}}{\Gamma_{1}}+\frac{f_{2}^{2}}{\Gamma_{2}}\right)\left[1-\frac{4}{\gamma \tau} \tanh \left(\frac{\gamma \tau}{4}\right)\right] .
\end{aligned}
$$

Novamente, uma vez que $(\gamma \tau) \geq 0$ e $(1-\tanh (x) / x) \geq 0$, temos que sempre $\bar{\Pi} \geq 0$. Note que a produção de entropia apresenta duas partes: a primeira relacionada com a diferença de temperaturas e a segunda dela dependente das forças externas.

\subsubsection{Força linear.}

Procedendo de forma análoga para uma força linear, as expressões para $\left\langle v_{1}\right\rangle(t)$ e $\left\langle v_{2}\right\rangle(t)$ são dadas por:

$$
\langle v\rangle=\left\{\begin{array}{c}
\left\langle v_{1}\right\rangle(t)=\frac{1}{\gamma}\left\{\lambda_{1}(\gamma t-1)+e^{-\gamma t}\left[\lambda_{1}+\left(\lambda_{2} e^{\frac{\gamma \tau}{2}}-\lambda_{1}\right) \alpha(\gamma, \tau)\right]\right\}, \\
\left\langle v_{2}\right\rangle(t)=\frac{1}{\gamma}\left\{-\lambda_{2}\left[\gamma\left(t-\frac{\tau}{2}\right)-1\right]+e^{-\gamma\left(t-\frac{\tau}{2}\right)}\left[\left(\lambda_{1} e^{\frac{\gamma \tau}{2}}-\lambda_{2}\right) \alpha(\gamma, \tau)-\lambda_{2}\right]\right\},
\end{array}\right.
$$

onde:

$$
\alpha(\gamma, \tau)=\frac{1-e^{\frac{\gamma \tau}{2}}\left(\frac{\gamma \tau}{2}-1\right)}{e^{\gamma \tau}-1}
$$

Uma vez que a energia interna de partícula não muda durante o período, dessa forma as expressões para o trabalho e calor são dadas por:

$$
\overline{\dot{W}}=\frac{m \tau \lambda_{1} \lambda_{2}}{24 \gamma \xi\left(e^{\gamma \tau}-1\right)}\left\{e^{\gamma \tau}\left[(\gamma \tau)^{2}-24 \xi\right]+12 e^{\frac{\gamma \tau}{2}} \xi(2+\gamma \tau)-\gamma \tau(\gamma \tau+6)-12\right\}
$$

$\mathrm{e}$

$$
\overline{\dot{Q}}=\frac{m \tau \lambda_{1} \lambda_{2}}{24 \gamma \xi\left(e^{\gamma \tau}-1\right)}\left\{-e^{\gamma \tau}[\gamma \tau(\gamma \tau+3)-48 \xi]-12 e^{\frac{\gamma \tau}{2}}[\xi(\gamma \tau+4)+2]+\gamma \tau(\gamma \tau+9)+24\right\}
$$

onde: $\xi=\left(\lambda_{1} \lambda_{2}\right) /\left(\lambda_{1}^{2}+\lambda_{2}^{2}\right)$. 


\subsection{Forma bilinear e coeficientes de Onsager.}

Uma vez que a produção de entropia é uma forma quadrática das forças $f_{T}, f_{1}, f_{2}$ e pode ser escrita como uma soma de fluxos e forças termodinâmicas:

$$
\bar{\Pi}=\mathcal{J}_{T} f_{T}+\tilde{L}_{11} f_{1}^{2}+\left(\tilde{L}_{12}+\tilde{L}_{21}\right) f_{1} f_{2}+\tilde{L}_{22} f_{2}^{2},
$$

onde a força $f_{T}=\left(1 / \Gamma_{1}-1 / \Gamma_{2}\right)$ e $f_{1(2)}$ está associada ao fluxo $\mathcal{J}_{T}$ na forma:

$$
\mathcal{J}_{T}=L_{T T} f_{T}
$$

pode-se identificar os elementos $\tilde{L}_{i j}$ como os coeficientes da matriz de Onsager.

No caso de força constante obtemos as seguintes expressões para os coeficientes de Onsager:

$$
\tilde{L}_{11}=\frac{1}{\Gamma_{1}}\left[1-\frac{3}{\gamma \tau} \tanh \left(\frac{\gamma \tau}{4}\right)\right]+\frac{1}{\gamma \tau \Gamma_{2}} \tanh \left(\frac{\gamma \tau}{4}\right)
$$

$\mathrm{e}$

$$
\tilde{L}_{12}+\tilde{L}_{21}=\frac{2}{\gamma \tau}\left(\frac{1}{\Gamma_{1}}+\frac{1}{\Gamma_{2}}\right) \tanh \left(\frac{\gamma \tau}{4}\right)
$$

Em que analogamente para o caso de força linear, encontramos as seguentes expressões para os coeficientes de Onsager:

$$
\begin{array}{r}
\tilde{L}_{11}=\frac{1}{\Gamma_{1}}\left[\frac{\gamma^{2} \tau^{2}}{12}-\frac{\gamma \tau\left(2 e^{\gamma \tau}+1\right)}{4\left(e^{\gamma \tau}-1\right)}+\frac{1}{1+e^{-\frac{\gamma \tau}{2}}}+\frac{1}{\gamma \tau} \tanh \left(\frac{\gamma \tau}{4}\right)\right]+ \\
+\frac{1}{\Gamma_{2}} \frac{\left[e^{\frac{\gamma \tau}{2}}(\gamma \tau-2)+2\right]^{2}}{4 \gamma \tau\left(e^{\gamma \tau}-1\right)}
\end{array}
$$

e

$$
\tilde{L}_{12}+\tilde{L}_{21}=\frac{\left(2 e^{\frac{\gamma \tau}{2}}-\gamma \tau-2\right)\left(2 e^{\frac{\gamma \tau}{2}}-\gamma \tau e^{\frac{\gamma \tau}{2}}-2\right)\left(\Gamma_{1}+\Gamma_{2}\right)}{2 \gamma \tau\left(e^{\gamma \tau}-1\right) \Gamma_{1} \Gamma_{2}}
$$

Para o caso de temperaturas iguais dos reservatórios, temos que a relação Eq.[3.37] torna-se:

$$
\bar{\Pi}=-\frac{2 \gamma}{\Gamma}\left(\bar{W}_{1}+\bar{W}_{2}\right)
$$

Portanto combinando as Eqs.[3.42-3.48] chegamos em:

$$
\bar{W}_{1}=-T \mathcal{J}_{1} f_{1}=L_{11} f_{1}^{2}+L_{12} f_{2} f_{1}
$$


e

$$
\bar{W}_{2}=-T \mathcal{J}_{2} f_{2}=L_{22} f_{2}^{2}+L_{21} f_{1} f_{2}
$$

respectivamente, de forma que os coeficientes de Onsager $L_{i j}^{\prime} s$ coincidem com os coeficientes $\tilde{L}_{i j}^{\prime} s$ quando $\Gamma_{1}=\Gamma_{2}=\Gamma$ (veja por exemplo Eqs.[3.33-3.34]).

\subsection{Caso assimétrico.}

Estenderemos aqui o problema para quando a partícula está em contato com dois reservatórios térmicos em diferentes intervalos de tempo para diferentes temperaturas. Assumimos primeiramente que a partícula browniana está em contato com o primeiro reservatório térmico num tempo $\left(\tau_{1}\right)$ e com o segundo um tempo $\left(\tau_{2}\right)$, com a condição $\tau_{1}+\tau_{2}=\tau$, em que novamente $(\tau)$ é o tempo total em que a partícula esta exposta pela ação das forças.

Conforme mostraremos no próximo capitulo, nossa motivação reside na construção da melhor máquina térmica ajustando sua assimetria e a diferença de temperaturas entre os reservatórios. Por tal, nós definiremos uma quantidade $(\kappa)$ que estabelece a razão entre os períodos de contato entre a partícula e cada reservatório $\kappa=\tau_{1} / \tau_{2}$, de forma que:

$$
\tau_{1}=\frac{\kappa \tau}{1+\kappa} \quad \text { e } \quad \tau_{2}=\frac{\kappa}{1+\kappa} .
$$

\subsubsection{Caso assimétrico sem forças externas.}

De forma análoga ao caso simétrico, procuramos calcular $\left\langle v_{i}\right\rangle(t)$ e a variância $b_{i}(t)$. Suas expressões são dadas por:

$$
b_{i}(t)=A_{i-1} e^{-2 \gamma_{i}\left(t-\tau_{i-1}\right)}+\frac{\Gamma_{i}}{2 \gamma_{i}},
$$

onde devido à periodicidade temos:

$$
b_{1}(0)=b_{2}(\tau) \quad, \quad b_{1}\left(\frac{\kappa \tau}{1+\kappa}\right)=b_{2}\left(\frac{\kappa \tau}{1+\kappa}\right),
$$

de forma que $b_{1}(t)$ e $b_{2}(t)$ são dados por:

$$
b_{1}(t)=-\frac{\left(\Gamma_{1}-\Gamma_{2}\right)\left(1-e^{-\frac{2 \gamma \tau}{1+\kappa}}\right)}{2 \gamma\left(1-e^{-2 \gamma \tau}\right)} e^{-2 \gamma t}+\frac{\Gamma_{1}}{2 \gamma} \quad ; \quad 0 \leq t<\frac{\kappa \tau}{1+\kappa}
$$


e

$$
b_{2}(t)=\frac{\left(\Gamma_{1}-\Gamma_{2}\right)\left(1-e^{-\frac{2 \gamma \kappa \tau}{1+\kappa}}\right)}{2 \gamma\left(1-e^{-2 \gamma \tau}\right)} e^{-2 \gamma\left(t-\frac{\kappa \tau}{1+\kappa}\right)}+\frac{\Gamma_{2}}{2 \gamma} \quad ; \quad \frac{\kappa \tau}{1+\kappa} \leq t<\tau .
$$

Neste caso, a produção de entropia deve-se somente à diferença de temperaturas e é dada por:

$$
\bar{\Pi}_{T}=\frac{1}{\tau}\left[\frac{2 \gamma^{2}}{\Gamma_{1}} \int_{0}^{\frac{\kappa \tau}{1+\kappa}} b_{1}(t) d t+\frac{2 \gamma^{2}}{\Gamma_{2}} \int_{\frac{\kappa \tau}{1+\kappa}}^{\tau} b_{2}(t) d t-2 \gamma\right] .
$$

Substituindo então Eqs.[3.54-3.55] em Eq.[3.56] e efetuando a integração obtemos:

$$
\bar{\Pi}_{T}=\frac{\Gamma_{1} \Gamma_{2}}{\tau} \frac{\sinh \left[\frac{\gamma \kappa \tau}{1+\kappa}\right] \sinh \left[\frac{\gamma \tau}{1+\kappa}\right]}{\sinh [\gamma \tau]}\left(\frac{1}{\Gamma_{1}}-\frac{1}{\Gamma_{2}}\right)^{2},
$$

onde é possível perceber que no caso $(\kappa=1)$ temos exatamente o mesmo resultado que no problema simétrico, então da bilinearidade da produção de entropia $\bar{\Pi}_{T}=L_{T T} f_{T}^{2}$, chegamos em:

$$
L_{T T}=\frac{\Gamma_{1} \Gamma_{2}}{\tau} \frac{\sinh \left[\frac{\gamma \kappa \tau}{1+\kappa}\right] \sinh \left[\frac{\gamma \tau}{1+\kappa}\right]}{\sinh [\gamma \tau]}
$$

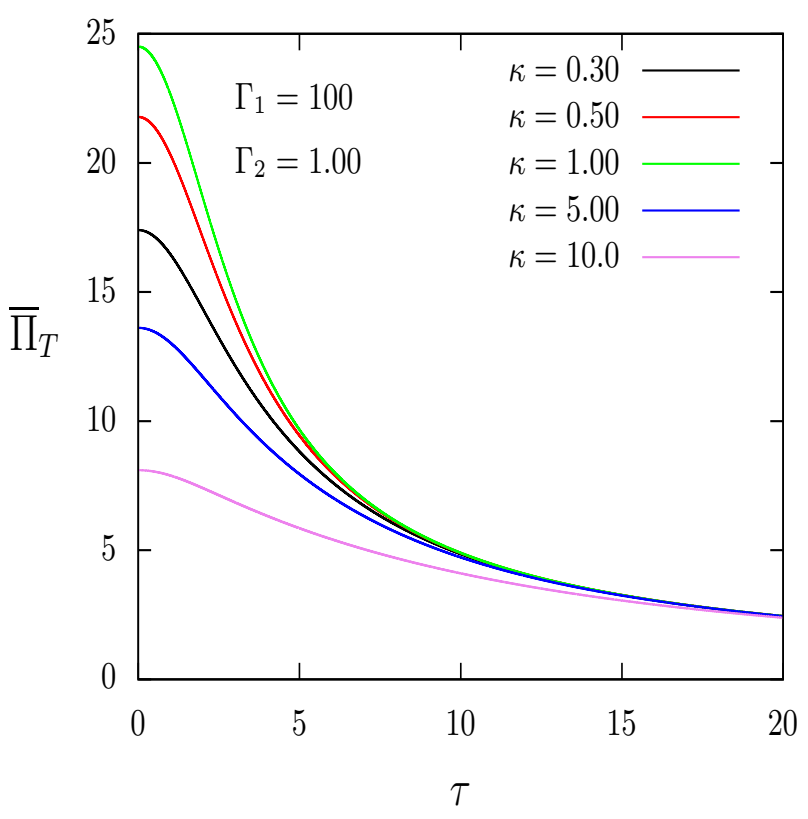

Figura 3.2: Produção de entropia versus o período para diferentes assimetrias $\kappa$ 's

Finalmente, vemos que a produção de entropia é sempre maior para o caso simétrico $(\kappa=1)$. 


\subsubsection{Caso assimétrico com forças externas.}

Da mesma forma que fizemos anteriormente, vamos considerar que cada partícula está sujeita a uma força em cada etapa. Neste caso, de forma análoga, a produção de entropia é a soma de dois termos, $\Pi=\Pi_{T}+\Pi_{F}$, sendo $\left(\Pi_{T}\right)$ dado pela Eq.[3.57]. Levando em vista que $\left\langle v_{i}^{2}\right\rangle(t)=\left\langle v_{i}\right\rangle^{2}(t)+b_{i}(t)$, a componente da produção de entropia devido as forças externas é dada por:

$$
\bar{\Pi}_{F}=\frac{1}{\tau}\left[\frac{2 \gamma^{2}}{\Gamma_{1}} \int_{0}^{\frac{\kappa \tau}{1+\kappa}}\left\langle v_{1}\right\rangle^{2}(t) d t+\frac{2 \gamma^{2}}{\Gamma_{2}} \int_{\frac{\kappa \tau}{1+\kappa}}^{\tau}\left\langle v_{2}\right\rangle^{2}(t) d t\right] .
$$

Da mesma forma fizemos anteriormente, vemos que:

$$
\bar{\Pi}_{F}=\tilde{L}_{11} X_{1}^{2}+\left(\tilde{L}_{12}+\tilde{L}_{21}\right) X_{1} X_{2}+\tilde{L}_{22} X_{2}^{2}
$$

então a partir Eq.[3.60] é possível calcular os elementos digonais $\left(\tilde{L}_{i i}, i=1,2\right)$ e a soma dos elementos cruzados da matriz de Onsager $\left(\tilde{L}_{i j}+\tilde{L}_{j i}, i \neq j\right)$, feito isso temos:

$$
\begin{gathered}
\tilde{L}_{11}=\frac{\cosh ^{2}\left(\frac{\gamma \tau}{2}\right)}{\gamma \tau \Gamma_{1} \Gamma_{2}(1+\kappa)}\left\{\gamma \Gamma_{2} \kappa \tau[\cosh (\gamma \tau)-1]+(1+\kappa)\left\{\left(\Gamma_{1}-2 \Gamma_{2}\right)\left[\cosh \left(\frac{\gamma \kappa \tau}{1+\kappa}\right)-1\right] \times\right.\right. \\
\left.\left.\quad \times \sinh \left(\frac{\gamma \tau}{1+\kappa}\right)-2 \Gamma_{2} \sinh ^{2}\left(\frac{\gamma \tau}{2(1+\kappa)}\right) \sinh \left(\frac{\gamma \kappa \tau}{1+\kappa}\right)\right\}\right\} \\
\tilde{L}_{22}=\frac{\cosh ^{2}\left(\frac{\gamma \tau}{2}\right)}{\gamma \tau \Gamma_{1} \Gamma_{2}(1+\kappa)}\left\{\gamma \Gamma_{1} \tau[\cosh (\gamma \tau)-1]-(1+\kappa)\left\{\Gamma_{1}\left[\cosh \left(\frac{\gamma \kappa \tau}{1+\kappa}\right)-1\right] \times\right.\right. \\
\left.\left.\quad \times \sinh \left(\frac{\gamma \tau}{1+\kappa}\right)+\left(\Gamma_{2}-2 \Gamma_{1}\right) \sinh ^{2}\left(\frac{\gamma \tau}{21+\kappa)}\right) \sinh \left(\frac{\gamma \kappa \tau}{1+\kappa}\right)\right\}\right\}
\end{gathered}
$$

$\mathrm{e}$

$$
\begin{aligned}
\tilde{L}_{12}+\tilde{L}_{21}=\frac{2 \cosh ^{2}\left(\frac{\gamma \tau}{2}\right)}{\gamma \tau \Gamma_{1} \Gamma_{2}}\left\{\Gamma _ { 2 } \left[\cosh \left(\frac{\gamma \kappa \tau}{1+\kappa}\right)\right.\right. & -1] \sinh \left(\frac{\gamma \tau}{1+\kappa}\right)+ \\
& \left.+2 \Gamma_{1} \sinh ^{2}\left(\frac{\gamma \tau}{2(1+\kappa)}\right) \sinh \left(\frac{\gamma \kappa \tau}{1+\kappa}\right)\right\}
\end{aligned}
$$


Novamente temos $\tilde{L}_{i j} \leftrightarrow \tilde{L}_{j i}$ trocando a ordem dos reservatórios e $\tau_{1} \leftrightarrow \tau_{2}$.

\section{Força Constante.}

Calculando de forma análoga as expressões para $\left\langle v_{1}\right\rangle(t)$ e $\left\langle v_{2}\right\rangle(t)$ chegamos em:

$$
\left\langle v_{1}\right\rangle(t)=\frac{f_{1}}{\gamma}+\frac{\left(f_{2}-f_{1}\right)}{\gamma} \frac{\left(e^{\frac{\gamma \tau}{1+\kappa}}-1\right)}{e^{\gamma \tau}-1} e^{-\gamma\left(t-\frac{\gamma \kappa \tau}{1+\kappa}\right)},
$$

e

$$
\left\langle v_{2}\right\rangle(t)=\frac{f_{2}}{\gamma}+e^{\frac{\gamma \tau}{1+\kappa}} \frac{\left(f_{1}-f_{2}\right)}{\gamma} \frac{\left(e^{\frac{\gamma \kappa \tau}{1+\kappa}}-1\right)}{e^{\gamma \tau}-1} e^{-\gamma\left(t-\frac{\gamma \tau}{1+\kappa}\right)},
$$

e para o caso de $\left\langle v_{1}^{2}\right\rangle(t)$ e $\left\langle v_{2}^{2}\right\rangle(t)$ temos:

$$
\begin{array}{r}
\left\langle v_{1}^{2}\right\rangle(t)=\frac{\Gamma_{1}}{2 \gamma}+e^{-2 \gamma t}\left\{\frac{\left(e^{\gamma t}-1\right) f_{1}}{\gamma}+\frac{\left(e^{\frac{\gamma \kappa \tau}{1+\kappa}}-1\right) f_{1}+e^{\frac{\gamma \kappa \tau}{1+\kappa}}\left(\frac{\gamma \tau}{1+\kappa}-1\right) f_{2}}{\gamma\left(e^{\gamma \tau}-1\right)}\right\}^{2}- \\
-\frac{e^{-2 \gamma\left(t-\frac{\gamma \kappa \tau}{1+\kappa}\right)}\left(e^{\frac{\gamma \kappa \tau}{1+\kappa}}-1\right)\left(\Gamma_{1}-\Gamma_{2}\right)}{2 \gamma\left(e^{2 \gamma \tau}-1\right)}
\end{array}
$$

e

$$
\begin{array}{r}
\left\langle v_{2}^{2}\right\rangle(t)=\frac{\Gamma_{2}}{2 \gamma}+e^{-2 \gamma\left(t-\frac{\gamma \kappa \tau}{1+\kappa}\right)}\left\{\frac{\left(e^{\gamma\left(t-\frac{\gamma \tau}{1+\kappa}\right)}-1\right) f_{2}}{\gamma}+\frac{e^{\gamma \tau} f_{1}-f_{2}+e^{\frac{\gamma \tau}{1+\kappa}}\left(f_{2}-f_{1}\right)}{\gamma\left(e^{\gamma \tau}-1\right)}\right\}^{2}- \\
-\frac{e^{-2 \gamma(t-\tau)}\left(e^{\frac{\gamma \kappa \tau}{1+\kappa}}-1\right)\left(\Gamma_{2}-\Gamma_{1}\right)}{2 \gamma\left(e^{2 \gamma \tau}-1\right)} .
\end{array}
$$

\section{Força Variável.}

Neste caso as expressões de $\left\langle v_{1}\right\rangle(t)$ e $\left\langle v_{2}\right\rangle(t)$ ficam:

$$
\begin{aligned}
\left\langle v_{1}\right\rangle(t)=\frac{1}{\gamma}\left\{\lambda_{1}[\gamma t-1]+\frac{e^{-\gamma t}}{e^{\gamma \tau}-1}\left\{\lambda_{1}\left[e^{\gamma \tau}+e^{\frac{\gamma \kappa \tau}{1+\kappa}}\left(\frac{\gamma \kappa \tau}{1+\kappa}-1\right)\right]-\right.\right. & \\
& \left.\left.-\lambda_{2}\left[e^{\frac{\gamma \kappa \tau}{1+\kappa}}+e^{\gamma \tau}\left(\frac{\gamma \tau}{1+\kappa}-1\right)\right]\right\}\right\},
\end{aligned}
$$

e

$$
\begin{aligned}
\left\langle v_{2}\right\rangle(t)=\frac{1}{\gamma}\left\{\lambda_{2}\left[1-\gamma\left(t-\frac{\kappa \tau}{1+\kappa}\right)\right]+\frac{e^{-\gamma\left(t-\frac{\kappa \tau}{1+\kappa}\right)}}{e^{\gamma \tau}}\{\right. & \lambda_{1}\left[e^{\frac{\gamma \tau}{1+\kappa}}+e^{\gamma \tau}\left(\frac{\gamma \kappa \tau}{1+\kappa}-1\right)\right]- \\
& \left.\left.-\lambda_{2}\left[e^{\frac{\gamma \tau}{1+\kappa}}\left(\frac{\gamma \tau}{1+\kappa}-1\right)+e^{\gamma \tau}\right]\right\}\right\},
\end{aligned}
$$


e para $\left\langle v_{1}^{2}\right\rangle(t)$ e $\left\langle v_{2}^{2}\right\rangle(t)$ chegamos em:

$$
\begin{array}{r}
\left\langle v_{1}^{2}\right\rangle(t)=e^{-2 \gamma t}\left\{\frac{\left[1+e^{\gamma t}(\gamma t-1)\right] \lambda_{1}}{\gamma}+\frac{\lambda_{1}\left(1+e^{\frac{\gamma \kappa \tau}{1+\kappa}}\right)\left(\frac{\gamma \kappa \tau}{1+\kappa}-1\right)-\lambda_{2}\left[e^{\frac{\gamma \kappa \tau}{1+\kappa}}+e^{\gamma \tau}\left(\gamma \tau-1-\frac{\gamma \kappa \tau}{1+\kappa}\right)\right]}{\gamma\left(e^{\gamma \tau}-1\right)}\right\}^{2}- \\
-\frac{e^{-2 \gamma\left(t-\frac{\gamma \kappa \tau}{1+\kappa}\right)}\left(e^{2 \frac{\gamma \kappa \tau}{1+\kappa}}-1\right)\left(\Gamma_{1}-\Gamma_{2}\right)}{2 \gamma\left(e^{2 \gamma \tau}-1\right)}+\frac{\Gamma_{1}}{2 \gamma}
\end{array}
$$

e

$$
\begin{aligned}
\left\langle v_{2}^{2}\right\rangle(t) & =e^{-2 \gamma\left(t-\frac{\gamma \kappa \tau}{1+\kappa}\right)}\left\{-\frac{\lambda_{2}\left[1+e^{\gamma\left(t-\frac{\kappa \tau}{1+\kappa}\right)}\left(\gamma \tau-1-\frac{\gamma \kappa \tau}{1+\kappa}\right)\right]}{\gamma}+\right. \\
& \left.+\frac{-\lambda_{2}+e^{\gamma \tau}\left(\frac{\gamma \kappa \tau}{1+\kappa}-1\right)+e^{\frac{\gamma \kappa \tau}{1+\kappa}}\left[\lambda_{1}+\lambda_{2}\left(1-\frac{\gamma \kappa \tau}{1+\kappa}\right)\right]}{\gamma\left(e^{\gamma \tau}-1\right)}\right\}^{2}+\frac{e^{-2 \gamma(t-\tau)}\left(e^{2 \frac{\gamma \kappa \tau}{1+\kappa}}-1\right)\left(\Gamma_{1}-\Gamma_{2}\right)}{2 \gamma\left(e^{2 \gamma \tau}-1\right)}+\frac{\Gamma_{2}}{2 \gamma}
\end{aligned}
$$

Portanto substituindo as relações Eqs.[3.64-3.71] em:

$$
\overline{\dot{W}}_{1}=-\frac{m f_{1}}{\tau} \int_{0}^{\frac{\kappa \tau}{1+\kappa}}\left\langle v_{1}\right\rangle(t) d t \quad, \quad \overline{\dot{W}}_{2}=-\frac{m f_{2}}{\tau} \int_{\frac{\kappa \tau}{1+\kappa}}^{\tau}\left\langle v_{2}\right\rangle(t) d t
$$

$\mathrm{e}$

$$
\overline{\dot{Q}}_{1}=\frac{m}{\tau} \int_{0}^{\frac{\kappa \tau}{1+\kappa}}\left(\gamma\left\langle v_{1}^{2}\right\rangle(t)-\frac{\Gamma_{1}}{2}\right) d t \quad, \quad \overline{\dot{Q}}_{2}=\frac{m}{\tau} \int_{\frac{\kappa \tau}{1+\kappa}}^{\tau}\left(\gamma\left\langle v_{2}^{2}\right\rangle(t)-\frac{\Gamma_{2}}{2}\right) d t,
$$

obtemos o trabalho e calor para ambos protocolos de força, garantindo a primeira lei da termodinâmica sobre o ciclo $\bar{W}_{1}+\bar{W}_{2}+\bar{Q}_{1}+\bar{Q}_{1}=0$. Maiores detalhes sobre os cálculos podem ser encontrados no apêndice A.4.

Portanto substituindo as expressões Eqs.[3.64-3.65] e Eqs.[3.68-3.69] em Eq.[3.72], obtemos diretamente os coeficientes de Onsager $L_{i j}$ para o caso de temperaturas iguais, onde para força constante chegamos em:

$$
\begin{gathered}
L_{12}=L_{21}=\frac{4 \operatorname{csch}\left(\frac{\gamma \tau}{2}\right) \sinh \left(\frac{\gamma \kappa \tau}{2}\right) \sinh \left(\frac{\gamma \tau}{2}\right)}{\gamma \Gamma \tau}, \\
L_{11}=\frac{2 \gamma \kappa}{\Gamma}-L_{12} \quad, \quad L_{22}=\frac{2 \gamma}{\Gamma}-L_{12},
\end{gathered}
$$

e no caso linear:

$$
\begin{aligned}
L_{11}=\frac{2}{\gamma \tau \Gamma\left(e^{\gamma \tau}-1\right)}\left\{\left(1+e^{\gamma \tau}\right)\right. & {\left[\left(\frac{\gamma \kappa \tau}{2(1+\kappa)}\right)^{2}\left(1+\frac{\gamma \kappa \tau}{3(1+\kappa)}\right)-1\right]+} \\
& \left.+e^{\frac{\gamma \tau}{2(1+\kappa)}}\left(1+\frac{\gamma \kappa \tau}{2(1+\kappa)}\right)+e^{\frac{\gamma \kappa \tau}{1+\kappa}}\left(1-\frac{\gamma \kappa \tau}{1+\kappa}\right)\right\}
\end{aligned}
$$




$$
\begin{gathered}
L_{22}=\frac{2}{\gamma \tau \Gamma\left(e^{\gamma \tau}-1\right)}\left\{\left(1+e^{\gamma \tau}\right)\left[\left(\frac{\gamma \tau}{1+\kappa}\right)^{2}\left(1+\frac{\gamma \tau}{3(1+\kappa)}\right)-1\right]+\right. \\
\left.+e^{\frac{\gamma \kappa \tau}{1+\kappa}}\left(1+\frac{\gamma \tau}{1+\kappa}\right)+e^{\frac{\gamma \tau}{1+\kappa}}\left(1-\frac{\gamma \tau}{1+\kappa}\right)\right\}, \\
L_{12}=\frac{2}{\gamma \tau \Gamma\left(e^{\gamma \tau}-1\right)}\left[1+\frac{\gamma \kappa \tau}{1+\kappa}-e^{\frac{\gamma \kappa \tau}{1+\kappa}}\right]\left[1-e^{\frac{\gamma \tau}{1+\kappa}}\left(1-\frac{\gamma \tau}{1+\kappa}\right)\right],
\end{gathered}
$$

$\mathrm{e}$

$$
L_{21}=\frac{2}{\gamma \tau \Gamma\left(e^{\gamma \tau}-1\right)}\left[1+\frac{\gamma \tau}{1+\kappa}-e^{\frac{\gamma \tau}{1+\kappa}}\right]\left[1-e^{\frac{\gamma \kappa \tau}{1+\kappa}}\left(1-\frac{\gamma \kappa \tau}{1+\kappa}\right)\right],
$$

portanto das relações acima é possível perceber em ambos casos que fazendo a troca da ordem dos reservatórios $\tau_{1} \leftrightarrow \tau_{2}$, obtemos $L_{11} \leftrightarrow L_{22}$ e $L_{12} \leftrightarrow L_{21}$. 


\section{CAPÍTULO 4}

\section{Rendimento}

\subsection{Introdução}

Uma das motivações dos primeiros trabalhos em termodinâmica foi a otimização das máquinas térmicas, ou seja, permitir que um sistema transforme a maior parte da energia que recebe em trabalho útil, permanecendo em limites físicos reais, ou seja que não toda a energia subministrada ao sistema pode-se transformar completamente em trabalho útil, mas procurar a menor dissipação possível. Embora as demandas mutuamente conflitantes na maximização da eficiência e da potência sejam especialmente ilustrativas em trabalhos da física atual, também comparamos outros critérios de otimização, como por exemplo, aqueles baseados na minimização da produção de entropia, já que ela está diretamente relacionada com a dissipação do sistema. Essa diversidade de critérios ilustra as sutilezas conceituais que surgem na termodinâmica dos tempos finitos. Além disso, as restrições de tempo devem ser incluídas em modelos realistas de motores para otimizar seu desempenho. 
Distintos trabalhos da física atual lidam com sistemas submetidos a forças periódicas que funcionam como máquinas térmicas [17],[18],[19],[20],[21],[22]. Neste trabalho ilustramos as ideias acima considerando uma máquina térmica browniana.

Neste caso temos diferentes possibilidades a considerar: considerando o mesmo período de interação, maximizar com relação a força e/ou período. Ou ainda, consideramos uma assimetria no intervalo de tempo em cada etapa e ainda maximizar com relação a força e/ou assimetria. 


\subsection{Rendimento para temperaturas iguais.}

Começando com o caso de temperaturas iguais $(\Delta \Gamma=0)$, também consideraremos $\bar{W}_{1} \leq$ 0 e $\mathcal{P}=\overline{\dot{W}}_{2} \geq 0$, garantindo assim a positividade do rendimento, que é definido na forma:

$$
\eta=-\frac{\overline{\dot{W}}_{2}}{\bar{W}_{1}}=-\frac{L_{22} X_{2}^{2}+L_{21} X_{2} X_{1}}{L_{12} X_{1} X_{2}+L_{11} X_{1}^{2}}
$$

e conjuntamente demostramos que neste caso a eficiência é justamente a razão entre os termos da produção de entropia.

Portanto a máquina térmica ótima é aquela de maior rendimento e potência, é importante avaliar o papel das forças $X_{2 m P}, X_{2 m E}$ e $X_{2 m S}$ (extremos que maximizam potência, rendimento e dissipação), onde quando o rendimento e a potência são máximos a dissipação é miníma [21]. Para o regime de temperaturas iguais, as quantidades $X_{2 m E}, X_{2 m P}$ ficam:

$$
X_{2 m E}=\frac{L_{11}}{L_{12}}\left(-1+\sqrt{1-\frac{L_{12} L_{21}}{L_{11} L_{22}}}\right) X_{1} \quad, \quad X_{2 m P}=-\frac{1}{2} \frac{L_{21}}{L_{22}} X_{1},
$$

portanto as a eficiência máxima e a eficiência a máxima potência estão dadas por:

$$
\eta_{m E}=-\frac{L_{21}}{L_{22}}+\frac{2 L_{11} L_{22}}{L_{12}^{2}}\left(1-\sqrt{1-\frac{L_{12} L_{21}}{L_{11} L_{22}}}\right) \quad, \quad \eta_{m P}=\frac{L_{21}^{2}}{4 L_{11} L_{22}-2 L_{12} L_{21}},
$$

respetivamente.

\subsubsection{Caso simétrico.}

Para o caso $(\kappa=1)$, os coeficientes de Onsager obtidos a partir das relações Eqs.[3.483.50] para o caso de força constante ficam:

$$
L_{11}=L_{22}=\frac{1}{\Gamma}-L_{12} \quad, \quad L_{12}=L_{21}=\frac{2\left(e^{\frac{\gamma \tau}{2}}-1\right)^{2}}{\gamma \tau \Gamma\left(e^{\gamma \tau}-1\right)},
$$

onde o sistema cumpre reciprocidade, da mesma forma é possível o cálculo dos coeficientes para caso de força linear, os quais ficam:

$$
\begin{aligned}
L_{11}=L_{22}=-\frac{1}{\gamma \Gamma \tau\left(e^{\gamma \tau}-1\right)}\{2- & \left(\frac{\gamma \tau}{2}\right)^{2}\left(1+\frac{\gamma \tau}{3}\right)-2 e^{\frac{\gamma \tau}{2}}\left(1+\frac{\gamma \tau}{2}\right)+ \\
& \left.+e^{\gamma \tau}\left[2-\left(\frac{\gamma \tau}{2}\right)^{2}\left(1-\frac{\gamma \tau}{3}\right)\right]-2 e^{\frac{\gamma \tau}{2}}\left(1-\frac{\gamma \tau}{2}\right)\right\},
\end{aligned}
$$




$$
\begin{aligned}
L_{12}=L_{21}=\frac{2}{\gamma \Gamma \tau\left(e^{\gamma \tau}-1\right)}\left\{\left(1+\frac{\gamma \tau}{2}\right)-e^{\frac{\gamma \tau}{2}}\right. & +e^{\gamma \tau}\left(1-\frac{\gamma \tau}{2}\right)- \\
& \left.-e^{\frac{\gamma \tau}{2}}\left(1+\frac{\gamma \tau}{2}\right)\left(1-\frac{\gamma \tau}{2}\right)\right\}
\end{aligned}
$$

As forças termodinâmicas que maximizam potência e eficiência neste caso são:

$$
X_{2 m P}=-\frac{L_{12}}{2 L_{11}} X_{1}, \quad X_{2 m E}=-\frac{L_{22}}{L_{12}}(1-\sqrt{1-q}) X_{1},
$$

respetivamente. Das relações acima é possível chegar às expressões do máximo do rendimento $\eta_{m E}$ e com máxima potência $\eta_{m P}$, onde no caso de $\mathcal{P}_{m E}, \mathcal{P}_{m P}$ acontece do mesmo jeito:

$$
\mathcal{P}_{m P}=\frac{\Gamma L_{22} q}{4} X_{1}^{2}, \quad \eta_{m P}=\frac{q}{2(2-q)}
$$

$\mathrm{e}$

$$
\mathcal{P}_{m E}=\frac{\Gamma L_{22} q \sqrt{1-q}}{(1+\sqrt{1-q})^{2}} X_{1}^{2}, \quad \eta_{m E}=\frac{q}{(1+\sqrt{1-q})^{2}},
$$

respetivamente, onde foi inserido o coeficiente de acoplamento:

$$
q(\gamma, \Gamma, \tau)=\frac{L_{12}^{2}}{L_{11} L_{22}}=\frac{\left(\Gamma L_{12}\right)^{2}}{\left(1-\Gamma L_{12}\right)^{2}}
$$




\subsubsection{Caso assimétrico.}

Realizando o mesmo procedimento no cálculo dos coeficientes, para $(\kappa \neq 1)$, chegamos as seguentes expressões para os coeficientes de Onsager considerando força constante e linear:

$$
L_{12}=L_{21}=\frac{2\left(e^{\frac{\gamma \tau}{1+\kappa}}-1\right)\left(e^{\frac{\gamma \kappa \tau}{1+\kappa}}-1\right)}{\gamma \tau \Gamma\left(e^{\gamma \tau}-1\right)}
$$

$\mathrm{e}$

$$
L_{11}=\frac{2}{\Gamma} \frac{\kappa}{1+\kappa}-L_{12} \quad, \quad L_{22}=\frac{2}{\Gamma} \frac{1}{1+\kappa}-L_{12},
$$

para a força constante e:

$$
\begin{aligned}
L_{11}=-\frac{1}{\gamma \Gamma \tau\left(e^{\gamma \tau}-1\right)}\{2- & \left(\frac{\gamma \kappa \tau}{1+\kappa}\right)^{2}\left(1+\frac{2}{3} \frac{\gamma \kappa \tau}{1+\kappa}\right)-2 e^{\frac{\gamma \tau}{1+\kappa}}\left(1+\frac{\gamma \kappa \tau}{1+\kappa}\right)+ \\
& \left.+e^{\gamma \tau}\left[2-\left(\frac{\gamma \kappa \tau}{1+\kappa}\right)^{2}\left(1-\frac{2}{3} \frac{\gamma \kappa \tau}{1+\kappa}\right)\right]-2 e^{\frac{\gamma \kappa \tau}{1+\kappa}}\left(1-\frac{\gamma \kappa \tau}{1+\kappa}\right)\right\},
\end{aligned}
$$

$$
\begin{aligned}
L_{22}=-\frac{1}{\gamma \Gamma \tau\left(e^{\gamma \tau}-1\right)}\{2- & \left(\frac{\gamma \tau}{1+\kappa}\right)^{2}-\frac{2}{3}\left(\frac{\gamma \tau}{1+\kappa}\right)^{3}-2 e^{\frac{\gamma \kappa \tau}{1+\kappa}}\left(1+\frac{\gamma \tau}{1+\kappa}\right)+ \\
& \left.+e^{\gamma \tau}\left[2-\left(\frac{\gamma \tau}{1+\kappa}\right)^{2}+\frac{2}{3}\left(\frac{\gamma \tau}{1+\kappa}\right)^{3}\right]-2 e^{\frac{\gamma \tau}{1+\kappa}}\left(1-\frac{\gamma \tau}{1+\kappa}\right)\right\}
\end{aligned}
$$

$$
\begin{aligned}
L_{12}=\frac{2}{\gamma \Gamma \tau\left(e^{\gamma \tau}-1\right)}\left\{\left(1+\frac{\gamma \kappa \tau}{1+\kappa}\right)-e^{\frac{\gamma \kappa \tau}{1+\kappa}}\right. & +e^{\gamma \tau}\left(1-\frac{\gamma \tau}{1+\kappa}\right)- \\
& \left.-e^{\frac{\gamma \tau}{1+\kappa}}\left(1+\frac{\gamma \kappa \tau}{1+\kappa}\right)\left(1-\frac{\gamma \tau}{1+\kappa}\right)\right\},
\end{aligned}
$$

$\mathrm{e}$

$$
\begin{aligned}
L_{21}=\frac{2}{\gamma \Gamma \tau\left(e^{\gamma \tau}-1\right)}\left\{\left(1+\frac{\gamma \tau}{1+\kappa}\right)-e^{\frac{\gamma \tau}{1+\kappa}}\right. & +e^{\gamma \tau}\left(1-\frac{\gamma \kappa \tau}{1+\kappa}\right)- \\
& \left.-e^{\frac{\gamma \kappa \tau}{1+\kappa}}\left(1+\frac{\gamma \tau}{1+\kappa}\right)\left(1-\frac{\gamma \kappa \tau}{1+\kappa}\right)\right\},
\end{aligned}
$$

para a força linear respetivamente. Note que enquanto $L_{12}=L_{21}$ para a força constante para todos os valores de $\kappa$, e $L_{12}=L_{21}$ apenas quando $\kappa=1$ no caso de força linear. 
De forma análoga, as quantidades máximas ficam na forma Eqs.[4.8-4.9], onde o coeficiente $(q)$ é:

$$
q(\Gamma, \kappa, \tau)=\frac{L_{12}^{2}}{L_{11} L_{22}}=\frac{\left(\Gamma L_{12}\right)^{2}}{\left(\frac{2 \kappa}{1+\kappa}-\Gamma L_{12}\right)\left(\frac{2}{1+\kappa}-\Gamma L_{12}\right)},
$$

Nas Figs.(4.1-4.2) mostramos para força constante e linear a eficiência, potência e produção de entropia nas diferentes assimetrias $\kappa^{\prime} s$.
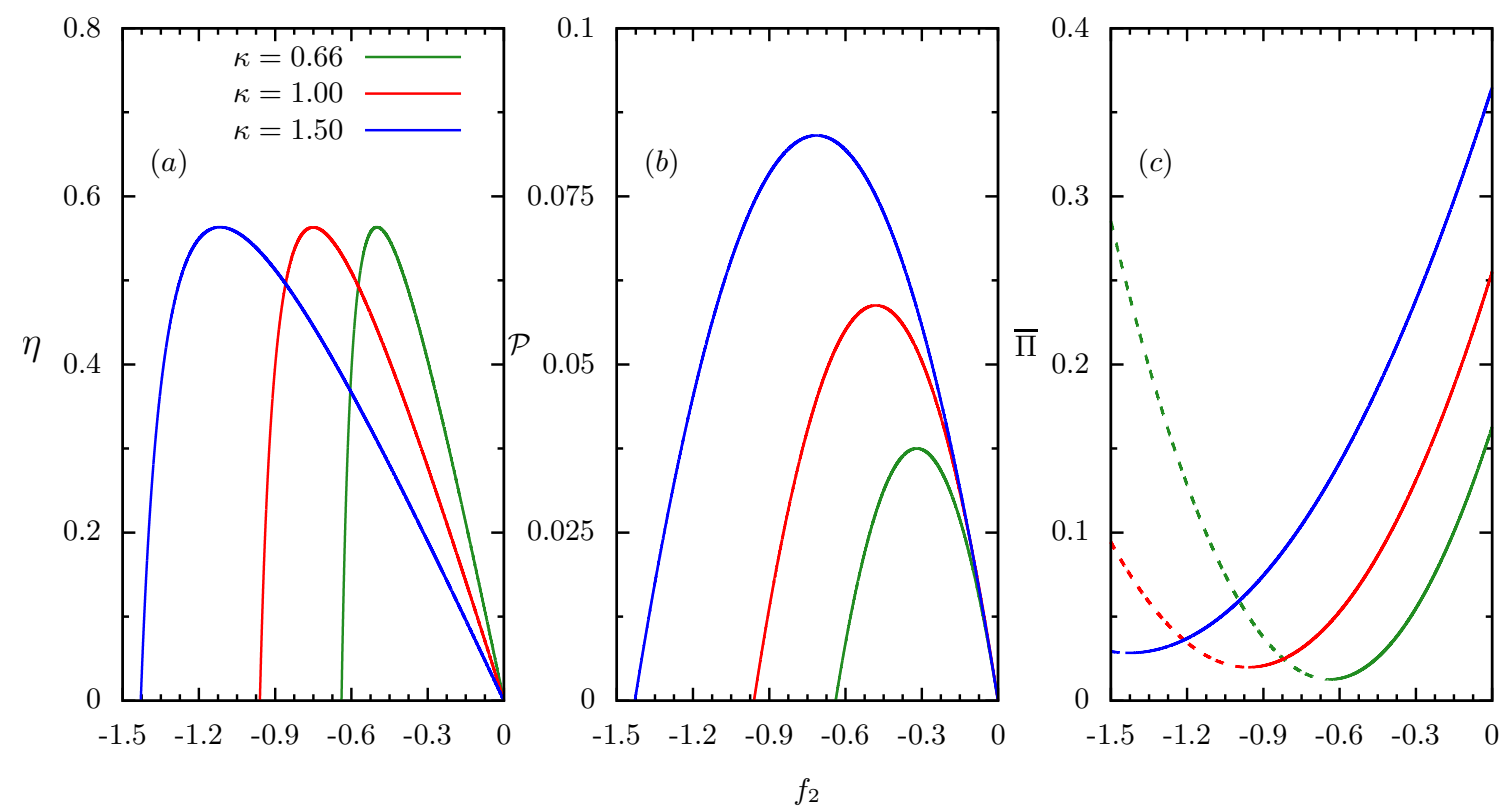

Figura 4.1: Painéis $(a),(b),(c)$ mostram eficiência $(\eta)$, potência $(\mathcal{P})$ e produção de entropia $(\bar{\Pi})$ respetivamente vs $f_{2}$ para diferentes assimetrias $\kappa$ 's, com $\left(\tau=f_{1}=1.0\right)$. Linhas tracejadas em $(c)$ mostram o regime não físico para o sistema operar como máquina térmica.

As Figs.(4.1-4.2) exemplificam as principais características dos tempos assimétricos para força constante e linear. Para $(\kappa>1)$ temos a maior potência e eficiência, consistente com que a partícula recebe energia durante um intervalo de tempo maior que a metade $\tau / 2$.

Para as Fig's.(4.3-4.4), no caso da potência a maximização com respeitos da força é mais eficiente que na assimetria, onde novamente o caso de força constante é mais eficiente que o caso linear. 

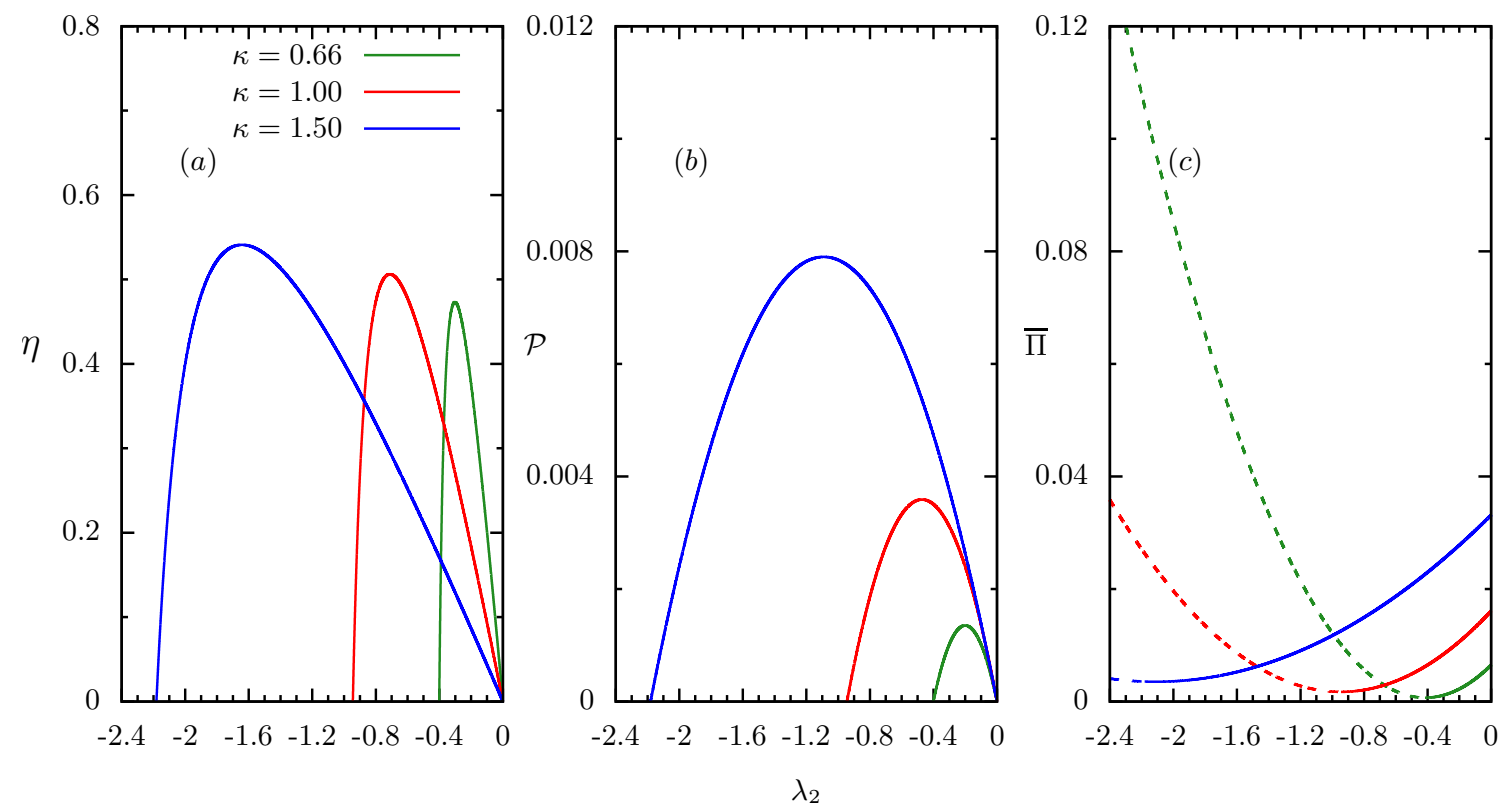

Figura 4.2: Painéis $(a),(b),(c)$ mostram eficiência $(\eta)$, potência $(\mathcal{P})$ e produção de entropia $(\bar{\Pi})$ respetivamente vs $\lambda_{2}$ para diferentes assimetrias $\kappa$ 's, com $(\tau=1.0) \mathrm{e}$ $\left(\lambda_{1}=-1.0\right)$. Linhas tracejadas em $(c)$ mostram o regímen não físico para o sistema operar como máquina térmica.
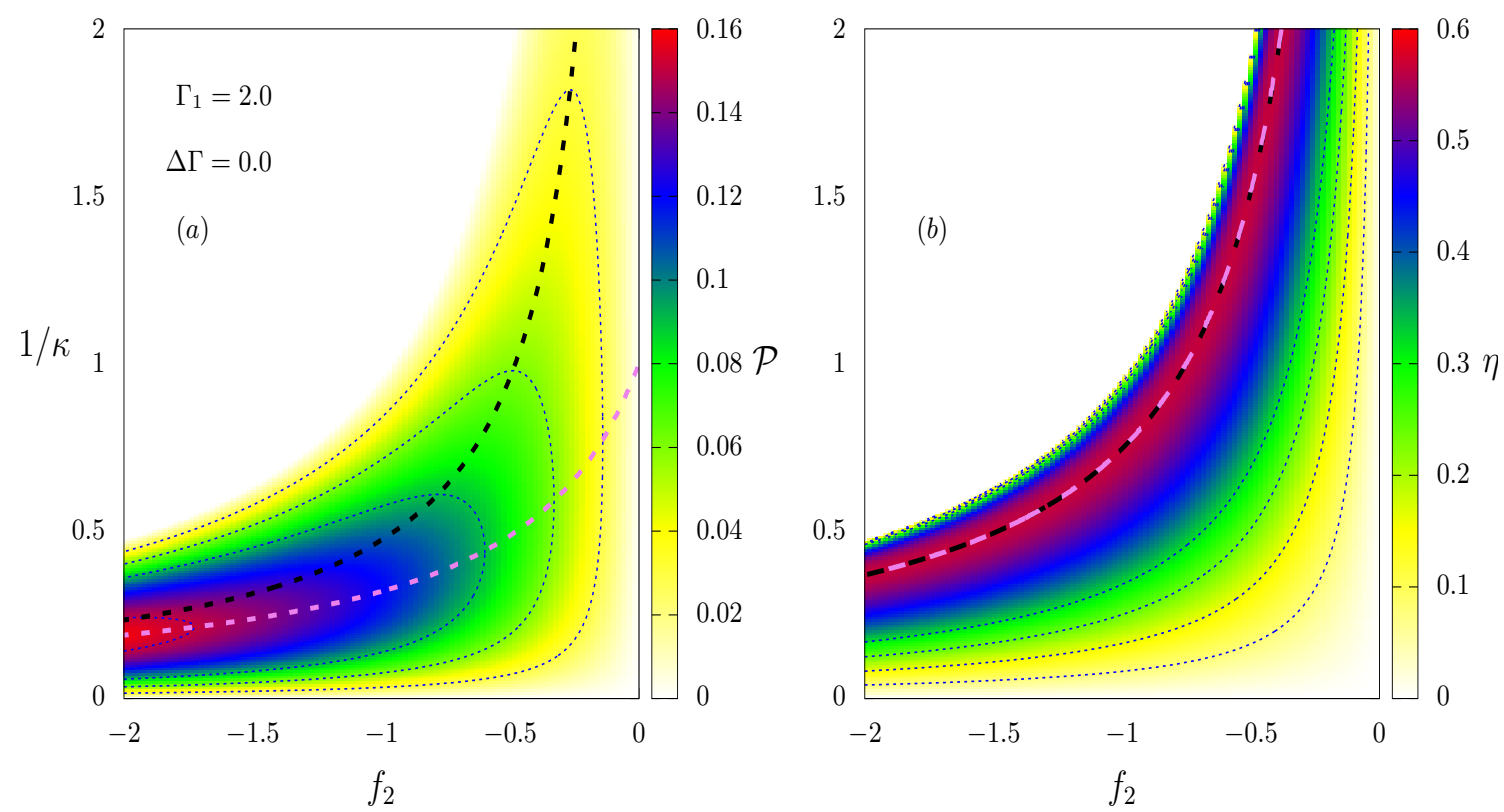

Figura 4.3: Painel $(a)$ mostram a potência $(\mathcal{P})$ e painel $(b)$ a eficiência $(\eta)$ em $\left(f_{2}, 1 / \kappa\right)$ $\operatorname{com}\left(\tau=f_{1}=1.0\right)$. Linhas tracejadas pretas e roxas mostram os máximos da potência e eficiência em $\left(f_{2}, 1 / \kappa\right)$ respetivamente. 

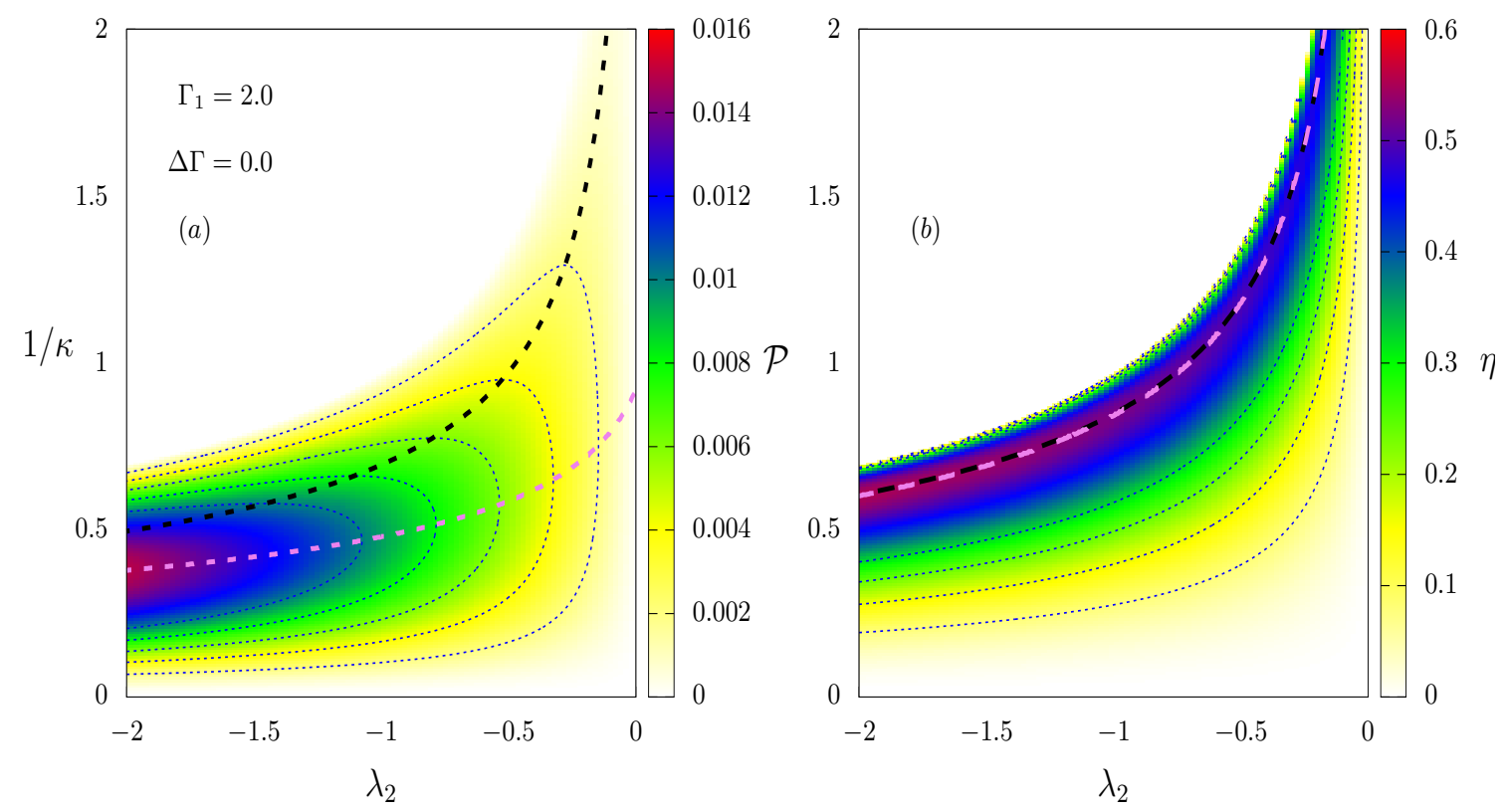

Figura 4.4: Painel $(a)$ mostram a potência $(\mathcal{P})$ e painel $(b)$ a eficiência $(\eta)$ em $\left(\lambda_{2}, 1 / \kappa\right)$ $\operatorname{com}(\tau=1.0)$ e $\left(\lambda_{1}=-1.0\right)$. Linhas tracejadas pretas e roxas mostram os máximos da potência e eficiência em $\left(\lambda_{2}, 1 / \kappa\right)$ respetivamente. 


\subsection{Temperaturas diferentes.}

Como extensão do problema, quando as temperaturas dos reservatórios são diferentes $\left(\Gamma_{1} \neq \Gamma_{2}\right)$, definimos a eficiência na forma:

$$
\eta=-\frac{\overline{\dot{W}}_{2}}{{\overline{\dot{W}_{1}}}_{1}+\overline{\dot{Q}}_{1}}
$$

onde $\bar{Q}_{1}$ corresponde ao calor recebido pela partícula divido ao reservatório. Note que a potência é a mesma do caso anterior.

\subsubsection{Caso simétrico.}

Procedendo de forma análoga ao apresentado anteriormente no cálculo dos coeficientes de Onsager e as quantidades máximas, aqui estudaremos o comportamento do sistema em relação as forças termodinâmicas e o período da máquina térmica.
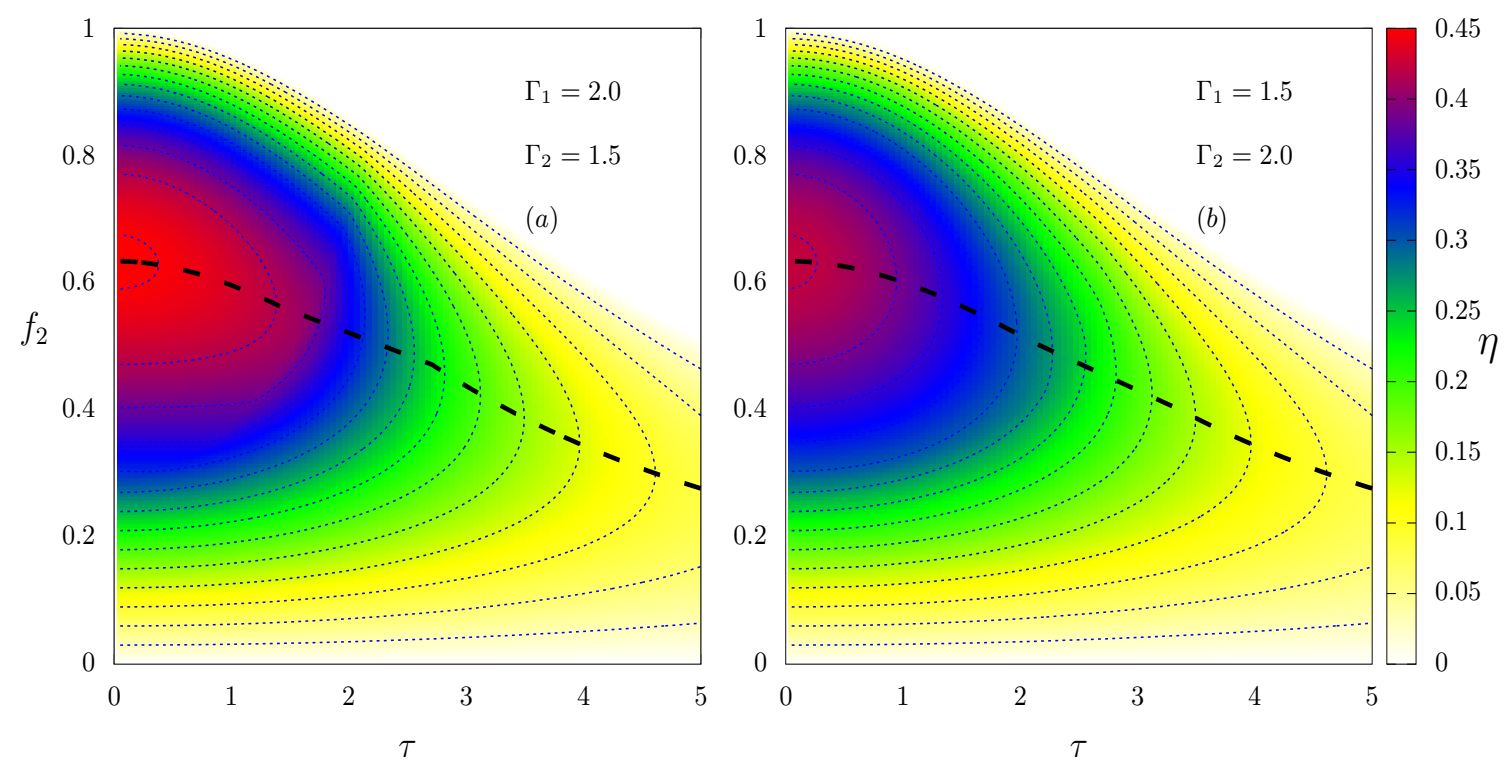

Figura 4.5: Painéis $(a)$ e $(b)$ mostram a eficiência em $\left(\tau, f_{2}\right)$ para $\left(f_{1}=-1.0\right)$. Linhas tracejadas pretas mostram a eficiência máxima em $f_{2}$.

Na Fig.(4.5) não foi feita a maximização com o período, pois períodos nulos maximizam a eficiência em ambos casos e isso fica fora da aplicabilidade da nossa máquina térmica, mas no caso $\left(\Gamma_{1}>\Gamma_{2}\right)$ temos temos maior rendimento. Para o caso da Fig.(4.6), 

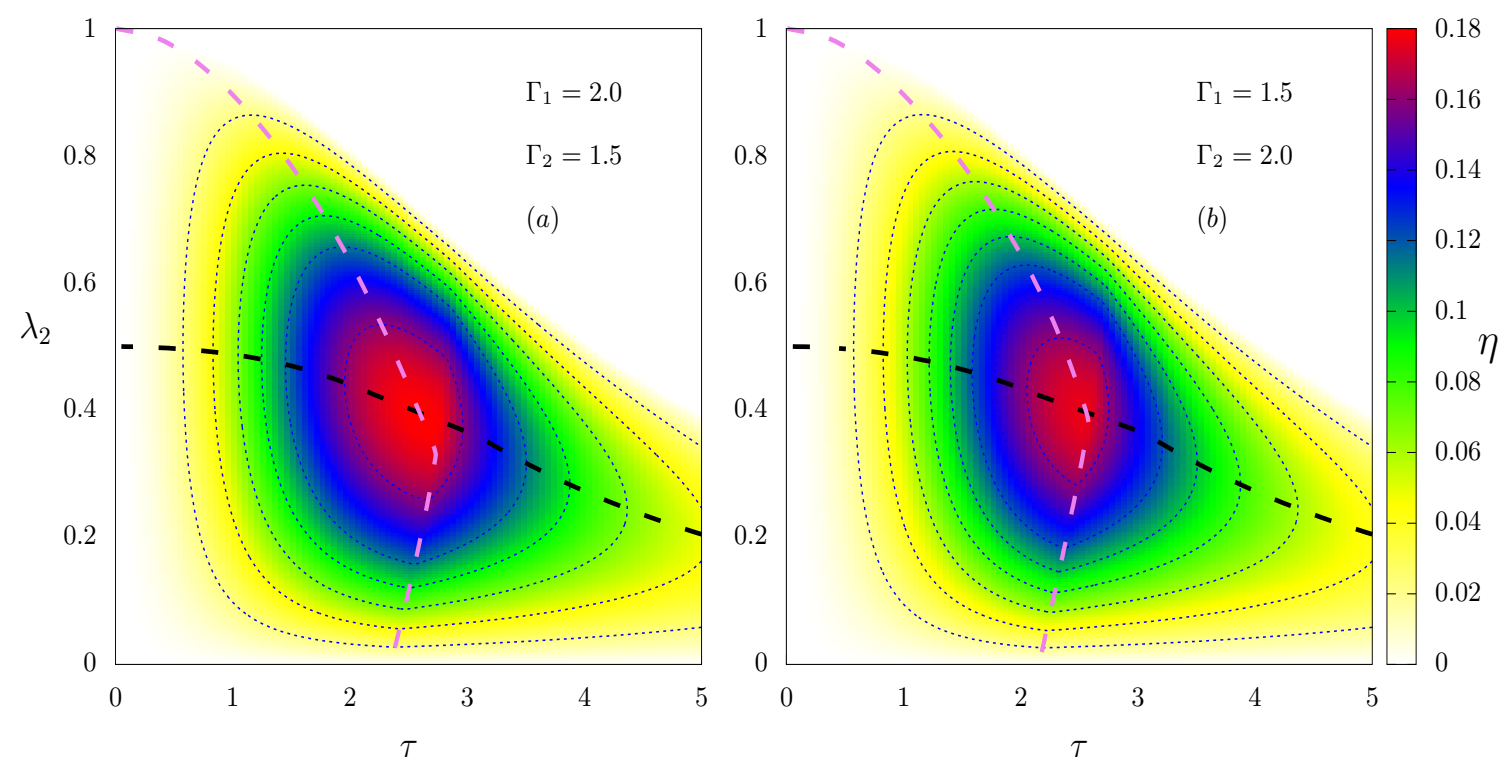

Figura 4.6: Painéis $(a)$ e $(b)$ mostram a eficiência em $\left(\tau, \lambda_{2}\right)$ para $\left(\lambda_{1}=1.0\right)$. Linhas tracejadas pretas e roxas mostram a eficiência máxima em $\left(\lambda_{2}, \tau\right)$ respetivamente.
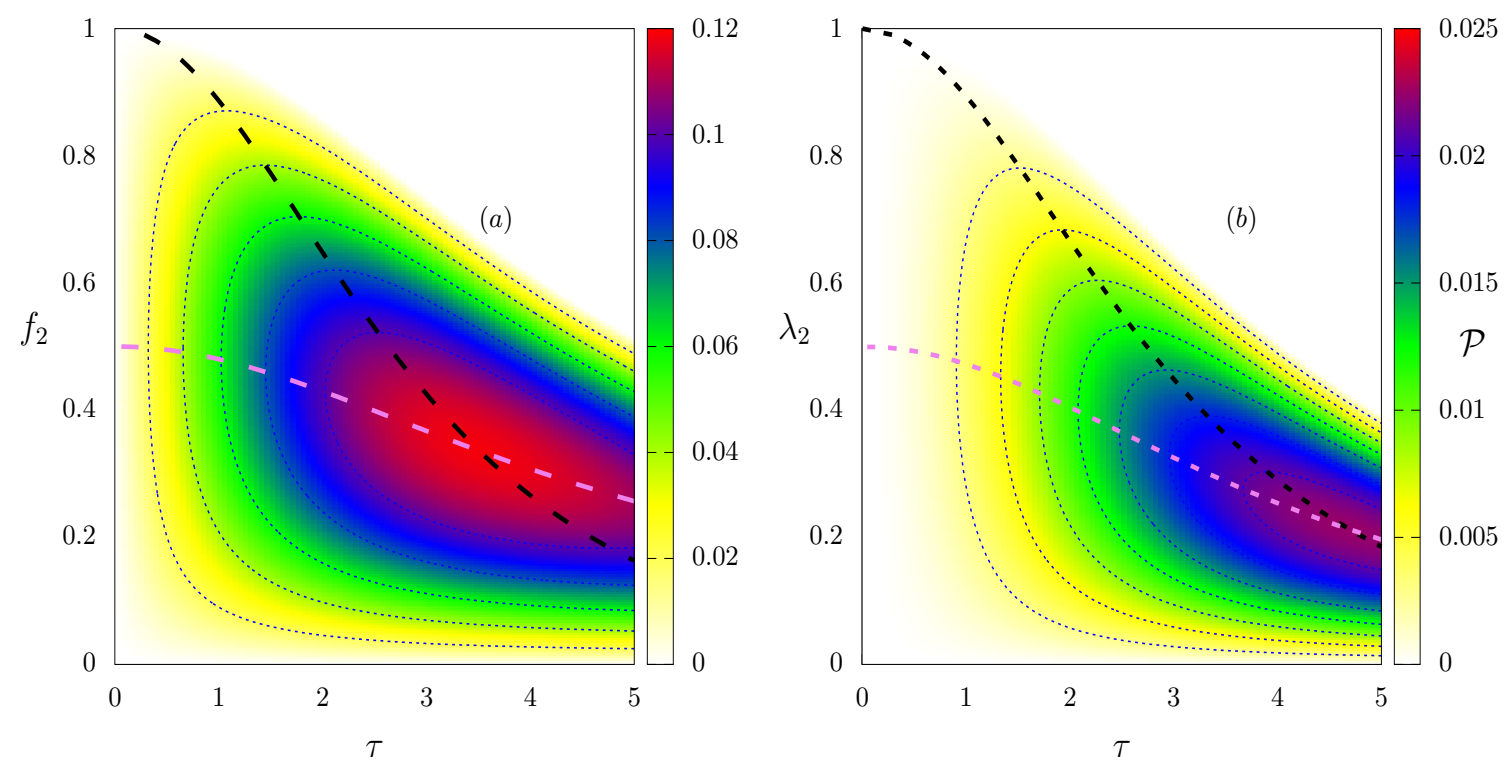

Figura 4.7: Painel $(a)$ mostra a potência em em $\left(\tau, f_{2}\right)$ para $\left(f_{1}=-1.0\right)$. Painel $(b)$ a potência em $\left(\tau, \lambda_{2}\right)$ para $\left(\lambda_{1}=1.0\right)$. Linhas tracejadas pretas e roxas mostram a potência máxima em $\left(f_{2}, \tau\right)$ e $\left(\lambda_{2}, \tau\right)$ respetivamente.

também quando $\left(\Gamma_{1}>\Gamma_{2}\right)$ a eficiência prevalece, onde o cruzamento das linhas pretas e roxas são o máximo global da mesma.

Comparando os paneis $(a)$ e $(b)$ da Fig.(4.7) notamos que para o caso de força constante a potência da máquina térmica é superior que no caso de força variável. 


\subsubsection{Caso assimétrico.}

Da mesma forma, mas quando $(\kappa \neq 1)$, apresentamos os resultados para o rendimento e potência no sistema, para ambos casos de forças (constante, linear), estudando o comportamento em relação as forças termodinâmicas e a assimetria.
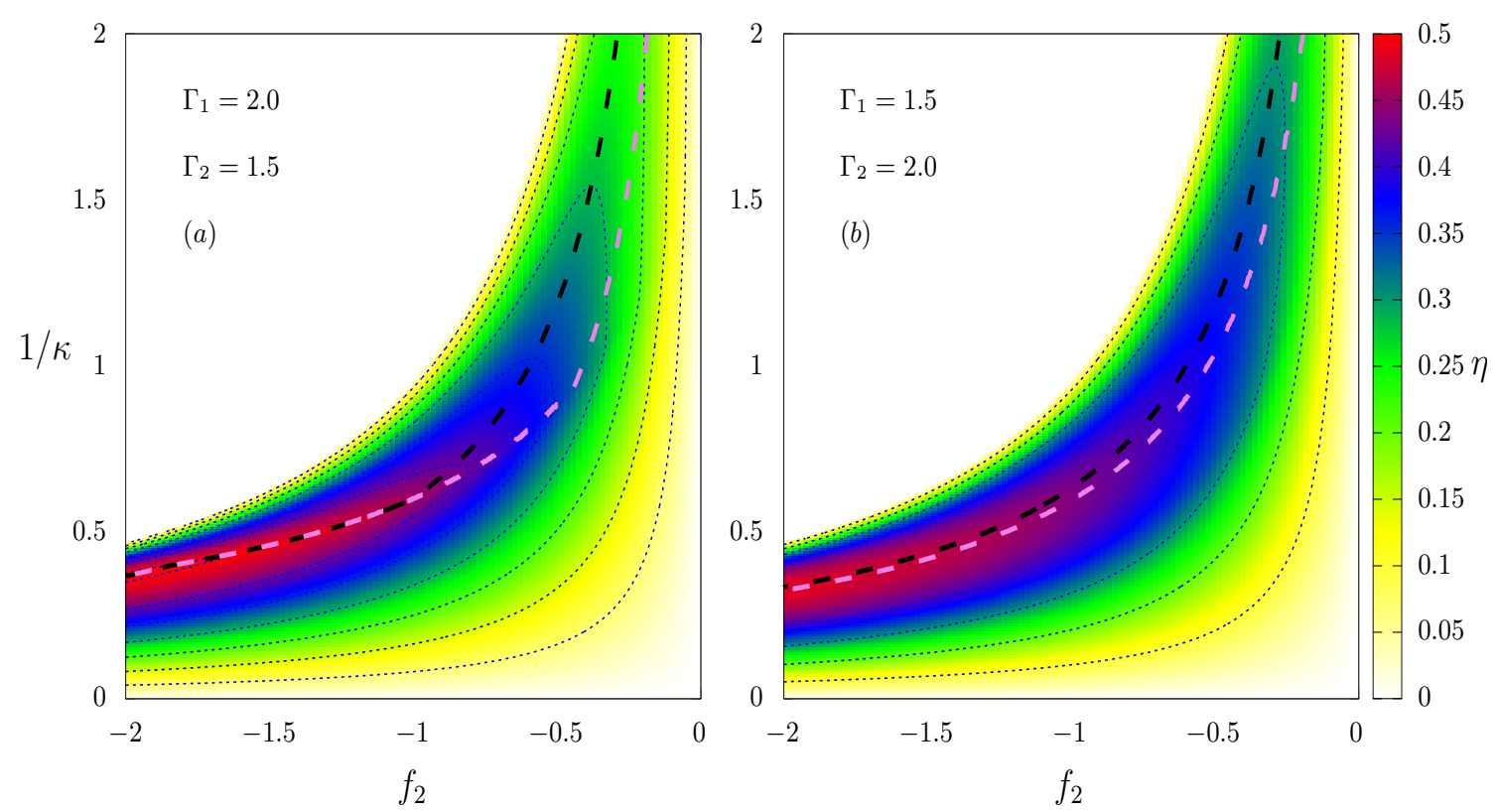

Figura 4.8: Painéis $(a)$ e $(b)$ mostram a eficiência em $\left(f_{2}, 1 / \kappa\right)$ para diferentes $\Delta \Gamma$ 's, com $\left(\tau=f_{1}=1.0\right)$. Linhas tracejadas pretas e roxas mostram a eficiência máxima em $\left(f_{2}, 1 / \kappa\right)$ respetivamente.

Nas fig.(4.8-4.9) percebamos que sempre o caso de força constante é superior, tanto na eficiência como na potência, também notamos que quando a partícula está mais tempo em contato com o reservatório quente o sistema é mais eficiente e em ambos casos a maximização com respeito às forças termodinâmicas torna-se superior em relação a assimetria. 

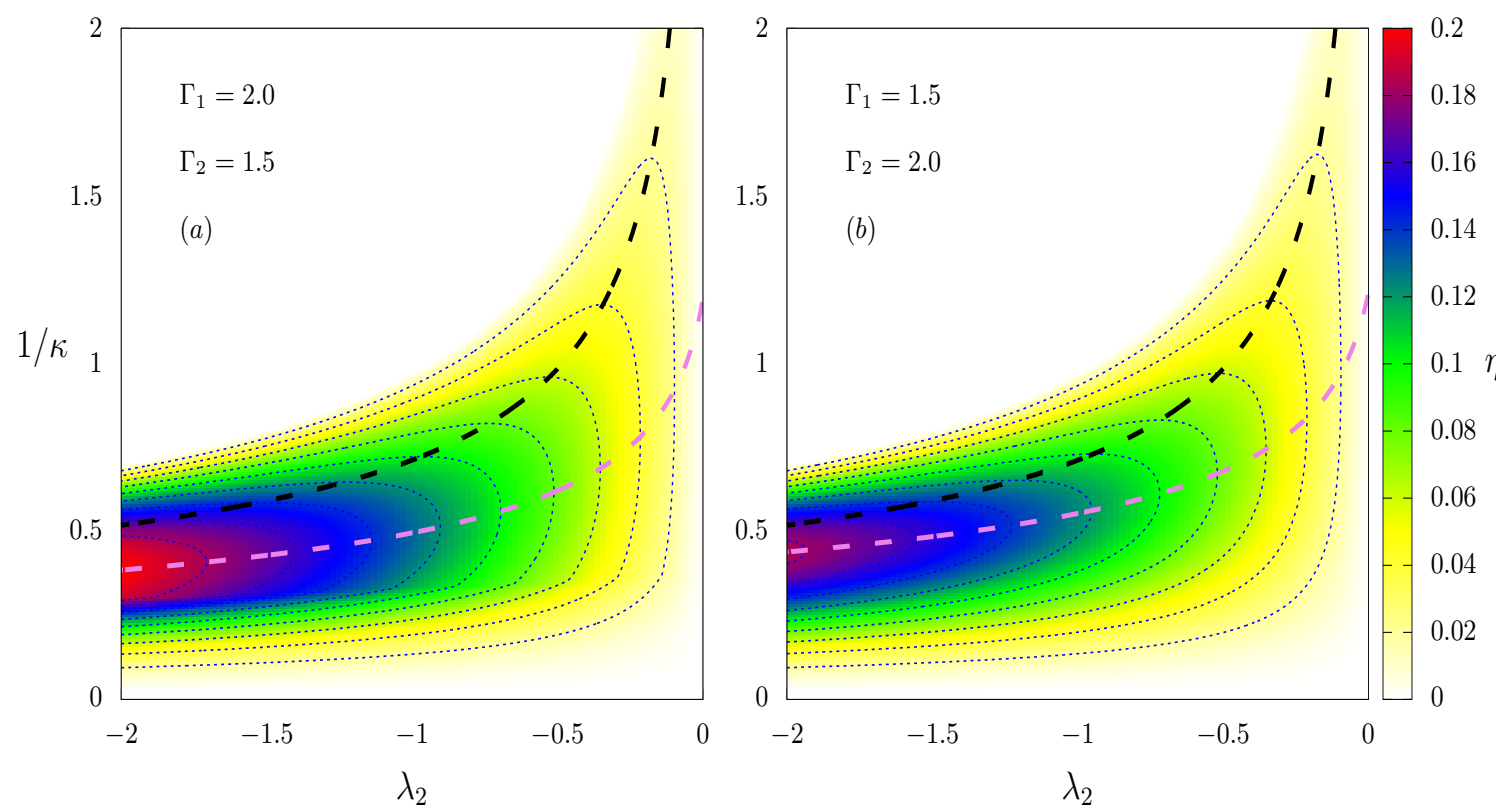

Figura 4.9: Painéis $(a)$ e $(b)$ mostram a eficiência em $\left(\lambda_{2}, 1 / \kappa\right)$ para diferentes $\Delta \Gamma$ 's, com $(\tau=1.0),\left(\lambda_{1}=-1.0\right)$. Linhas tracejadas pretas e roxas mostram a eficiência máxima em $\left(\lambda_{2}, 1 / \kappa\right)$ respetivamente. 


\subsection{Descrição aproximada.}

Considerando uma força genérica $X_{1}$, associada ao fluxo $\mathcal{J}_{1} X_{1} \leq 0$, além das forças $X_{2}$ e $X_{T}$, com um fluxo na forma $\mathcal{J}_{2} X_{2}+\mathcal{J}_{T} X_{T} \geqslant 0$, definimos, num primeiro momento deste projeto o rendimento da seguente forma:

$$
\eta=-\frac{\mathcal{J}_{1} X_{1}}{\mathcal{J}_{2} X_{2}+\mathcal{J}_{T} X_{T}}=-\frac{L_{11} X_{1}^{2}+L_{12} X_{1} X_{2}}{L_{21} X_{2} X_{1}+L_{22} X_{2}^{2}+L_{T T} X_{T}^{2}},
$$

onde na relação de acima para o caso $(\Delta \Gamma=0)$, se reduz a razão entre os trabalhos Eq.[4.1]. A expressão acima teve como motivação o fato de que uma força $X_{1}$ para "gerar"trabalho útil deve ser oposta as forças de "driving" $X_{2}$ e $X_{T}$, de forma que ela para um fluxo $\mathcal{J}_{1} X_{1}<0$ contrário ao $\mathcal{J}_{2} X_{2}$ e $\mathcal{J}_{T} X_{T}$.

Procedendo de forma análoga, as quantidades que maximizam rendimento e potência ficam:

$$
2 X_{1 m P}=-L_{12} X_{2} / L_{11} \quad, \quad X_{1 m S}=-L_{12} X_{2} / L_{11}=2 X_{1 m P}
$$

$\mathrm{e}$

$$
X_{1 m E}=\frac{1}{L_{11} L_{12} X_{2}}\left[-L_{11}\left(L_{22} X_{2}^{2}+L_{T T} X_{T}^{2}\right)+A\left(X_{2}, X_{T}\right)\right],
$$

onde $A\left(X_{2}, X_{T}\right)$ tem a forma:

$$
A\left(X_{2}, X_{T}\right)=\sqrt{L_{11}\left[L_{22} X_{2}^{2}+L_{T T} X_{T}^{2}\right]\left[L_{11}\left(L_{22} X_{2}^{2}+L_{T T} X_{T}^{2}\right)-L_{12}^{2} X_{2}^{2}\right]},
$$

portanto, o rendimento para máxima dissipação, potência máxima e o rendimento máximo ficam: $\eta_{m S}=0$,

$$
\eta_{m P}=\frac{L_{12}^{2} X_{2}^{2}}{2\left(2 L_{22} L_{11}-L_{12}^{2}\right) X_{2}^{2}+4 L_{T T} L_{11} X_{T T}^{2}},
$$

$\mathrm{e}$

$$
\eta_{m E}=\frac{1}{L_{12}^{2} X_{2}^{2}}\left[2 L_{11}\left(L_{22} X_{2}^{2}+L_{T T} X_{T T}^{2}\right)-L_{12}^{2} X_{2}^{2}-2 A\left(X_{2}, X_{T}\right)\right]
$$

avaliando as quantidades máximas na potência temos: $\mathcal{P}_{m S}=0, \mathcal{P}_{m P}=\Gamma_{1} L_{12}^{2} X_{2}^{2} / 4 L_{11}$ $\mathrm{e}$

$$
\begin{aligned}
\mathcal{P}_{m E} & =\frac{\Gamma_{1}}{L_{11} L_{12}^{2} X_{2}^{2}} \times\left[L_{11}\left(L_{22} X_{2}^{2}+L_{T T} X_{T}^{2}\right)-A\left(X_{2}, X_{T}\right)-L_{12}^{2} X_{2}^{2}\right] \times \\
& \times\left[L_{11}\left(L_{22} X_{2}^{2}+L_{T T} X_{T}^{2}\right)-A\left(X_{2}, X_{T}\right)\right]
\end{aligned}
$$

respectivamente. 
Assim as expressões mostradas anteriormente concordam com os resultados de [21] para o caso de uma partícula com duas forças atuando sobre ela, também a potência e eficiência são nula quando $\left(X_{1}=X_{1 m s}=0\right)$. Além disso elas são estritamente positivas entre esses limites. Assim como o rendimento tem que ser sempre positivo, isso impõe um limite físico aceitável para que o sistema possa operar como máquina térmica, que ao mesmo tempo está dentro dos limites da produção de entropia:

$\bar{\Pi}_{m S}=L_{T T} X_{T}^{2}+\left(L_{22}-L_{12}^{2} / L_{11}\right) X_{2}^{2} \quad, \quad \bar{\Pi}^{*}=L_{T T} X_{T}^{2}+L_{22} X_{2}^{2} \quad, \quad \bar{\Pi} \in\left[\bar{\Pi}_{m S}, \bar{\Pi}^{*}\right]$,

também é importante a relação entre as potências $\mathcal{P}_{m P}, \mathcal{P}_{m E}$ e as eficiências $\eta_{m P}, \eta_{m E}$, que são verificadas de acordo com [20],[21], então temos:

$$
\eta_{m P}=\frac{\eta_{m E}}{1+\eta_{m E}^{2}} \quad, \quad \frac{\mathcal{P}_{m E}}{\mathcal{P}_{m P}}=1-\eta_{m E}^{2},
$$

onde as relações Eq.[4.27] implicam as seguentes restrições:

$$
0 \leq \eta_{m P} \leq \eta_{m E}, \quad 0 \leq \eta_{m E} \leq 1, \quad 0 \leq \eta_{m P} \leq 1 / 2, \quad 0 \leq \mathcal{P}_{m E} \leq \mathcal{P}_{m P}
$$

Assim a máquina térmica mais eficiente $\left(\eta_{m E}=1\right)$ implica que o sistema tem que operar com potência de máxima eficiência nula $\left(\mathcal{P}_{m E}=0\right)$. No regime de $\tau \ll 1$, temos que:

$$
\eta_{m P} \rightarrow \frac{f_{2}^{2}\left(\Gamma_{1}+\Gamma_{2}\right)}{2\left[f_{2}^{2}\left(\Gamma_{1}+\Gamma_{2}\right)+2 \Delta \Gamma^{2}\right]}
$$

No caso de temperaturas muito próximas entre os reservatórios $\left(\Gamma_{1} \approx \Gamma_{2}\right)$ as expressões calculadas anteriormente ficam: $\eta_{m P} \rightarrow 1 / 2, \eta_{m E} \rightarrow 1 \mathrm{e} \mathcal{P}_{m P} \rightarrow f_{2}^{2} / 8$.

Para quando a força é linear é também verificado um aumento da eficiência e no caso de períodos pequenos e temperaturas muito próximas. Além disso, para diferença de temperaturas finitas, força constante é sempre mais eficiente que para força linear. Conclusões similares verificamos para a potência. Entretanto, para a máquina térmica com força linear $\eta_{m P}, \eta_{m E}, \mathcal{P}_{m E}$ e $\mathcal{P}_{m P}$ apresentam comportamentos reentrantes com o período.

Em suma, apresentamos um estudo para a eficiência da máquina térmica browniana qualitativo, onde na Fig.(4.13) é importante destacar que a definição apresentada acima super-estima os valores de eficiência, mas está em concordância qualitativa com a definição dada pela Eq.[4.18]. 

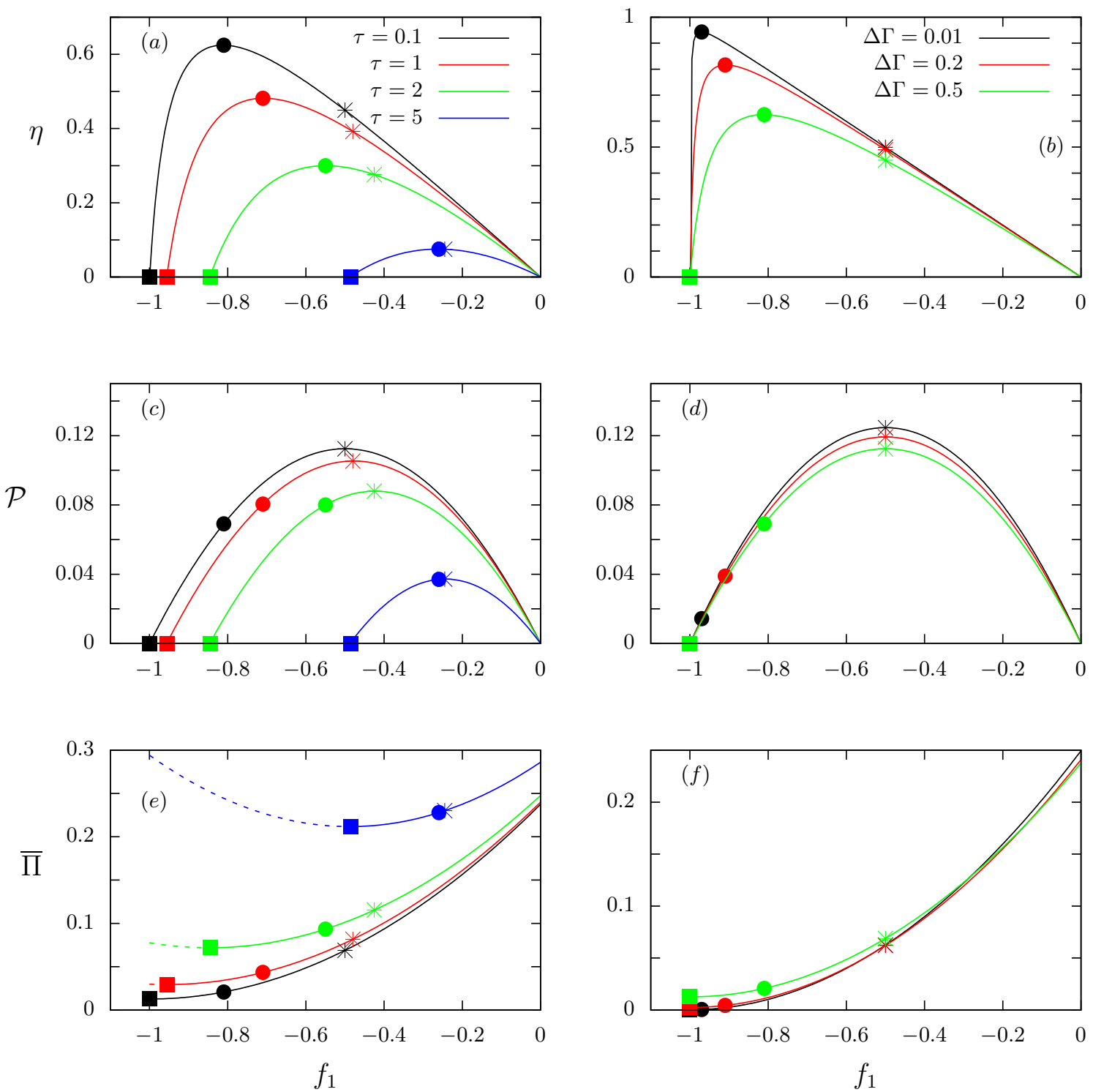

Figura 4.10: Painéis $(a)$ e $(b)$ representam a eficiência $\eta$ versus $f_{1}$ para diferentes assimetrias $(\kappa$ 's $),(\Delta \Gamma$ 's) respectivamente e $(\tau=1)$. Paneis da esquerda mostram $\left(\Gamma_{1}=2\right)$ e $\left(\Gamma_{2}=1.5\right)$, paneis da direita o caso contrario. Paneis $(c)$ e $d$ mostram a potência, enquanto paneis $(e)$ e $(f)$ produção de entropia nos mesmos parâmetros, linhas tracejadas em $(e)$ e $(f)$ mostram o regímen não físico da produção de entropia. 

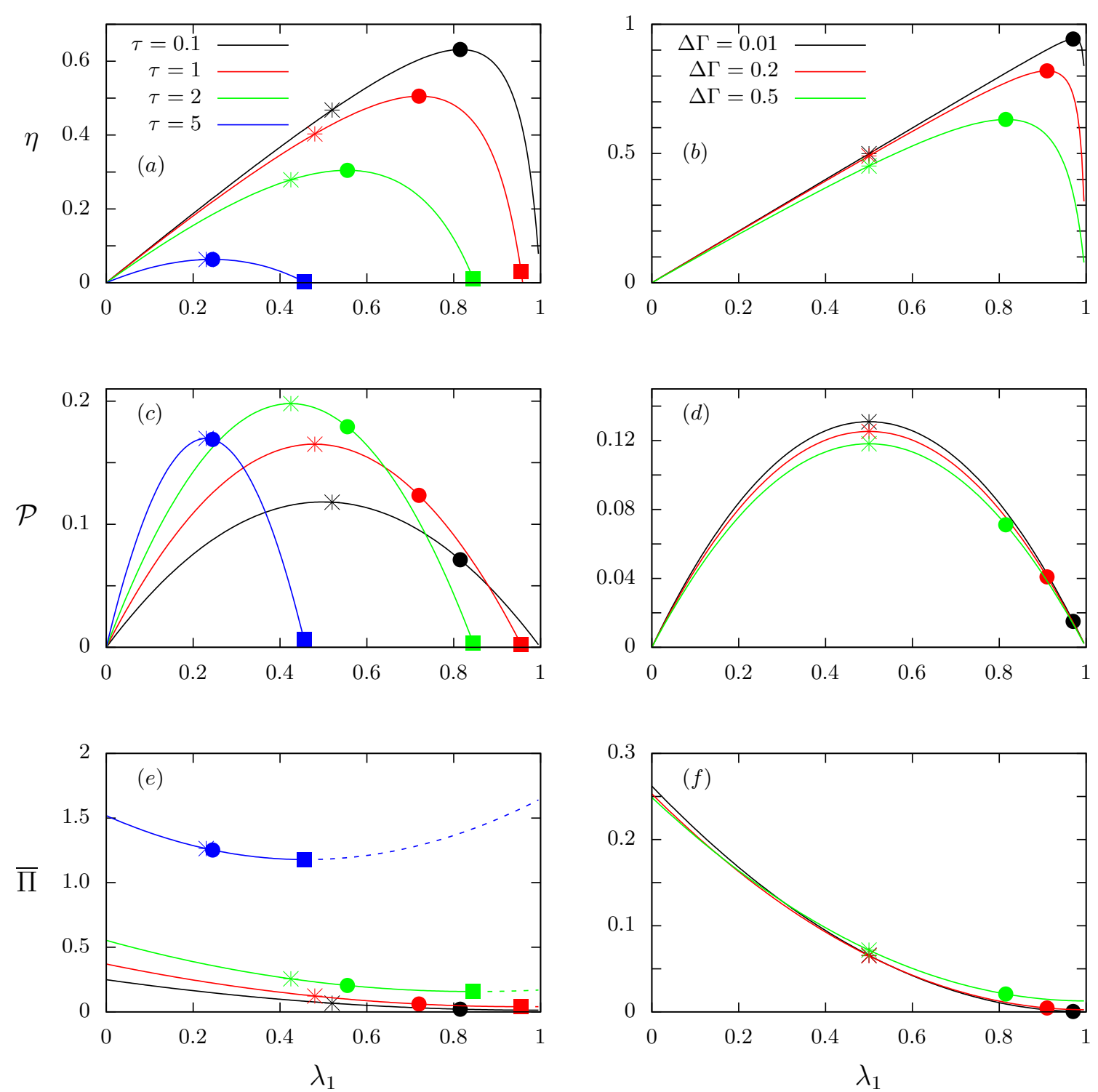

Figura 4.11: Painéis $(a)$ e $(b)$ representam a eficiência $\eta$ versus $\lambda_{1}$ para diferentes assimetrias $(\kappa$ 's $),\left(\Delta \Gamma^{\prime}\right.$ s) respectivamente e $(\tau=1)$. Paneis da esquerda mostram $\left(\Gamma_{1}=2\right) \quad e \quad\left(\Gamma_{2}=1.5\right)$, paneis da direita o caso contrario. Paneis $(c)$ e $d$ mostram a potência, enquanto paneis $(e)$ e $(f)$ produção de entropia nos mesmos parâmetros, linhas tracejadas em $(e)$ e $(f)$ mostram o regímen não físico da produção de entropia. 


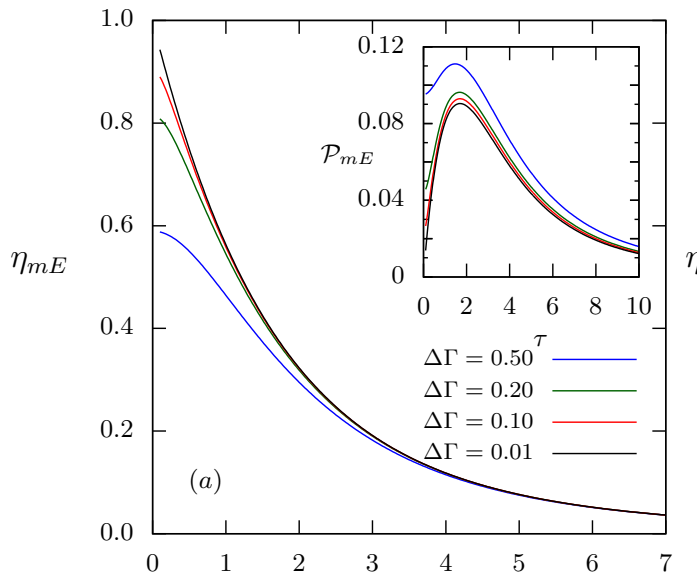

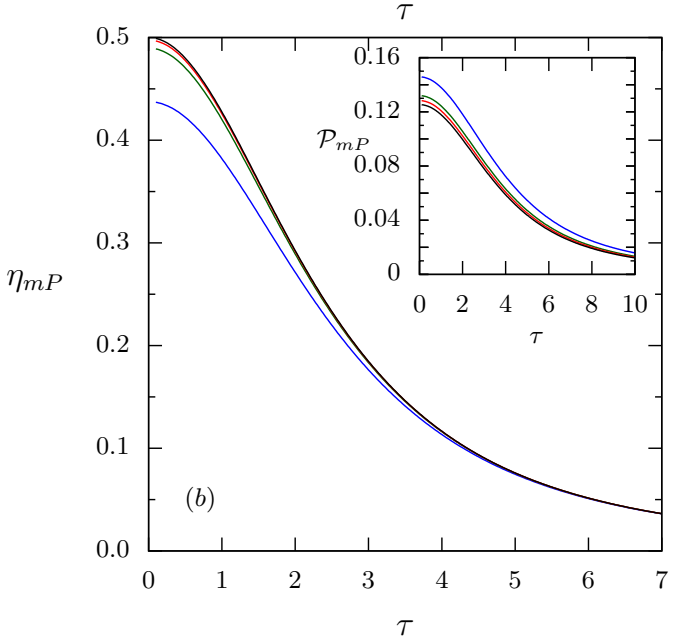

(a) Força Constante
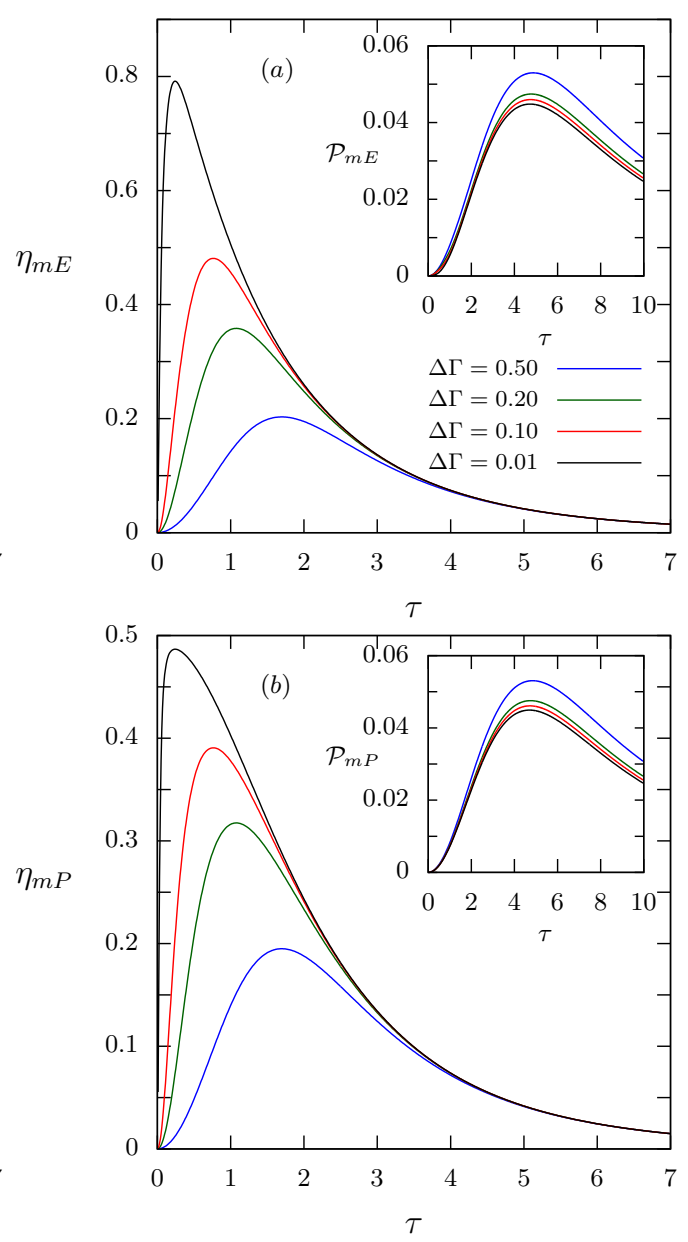

(b) Força Linear

Figura 4.12: Para $\Gamma_{1}=2,\left(f_{2}, \lambda_{2}\right)=1$ com diferentes valores $\Delta \Gamma$ 's, comparamos o rendimento máximo nos painéis $(a),(c)$ e o rendimento com máxima potência em painéis $(b),(d)$. Sub-figuras correspondem à potência máxima $\mathcal{P}$ 's versus $\tau$. 

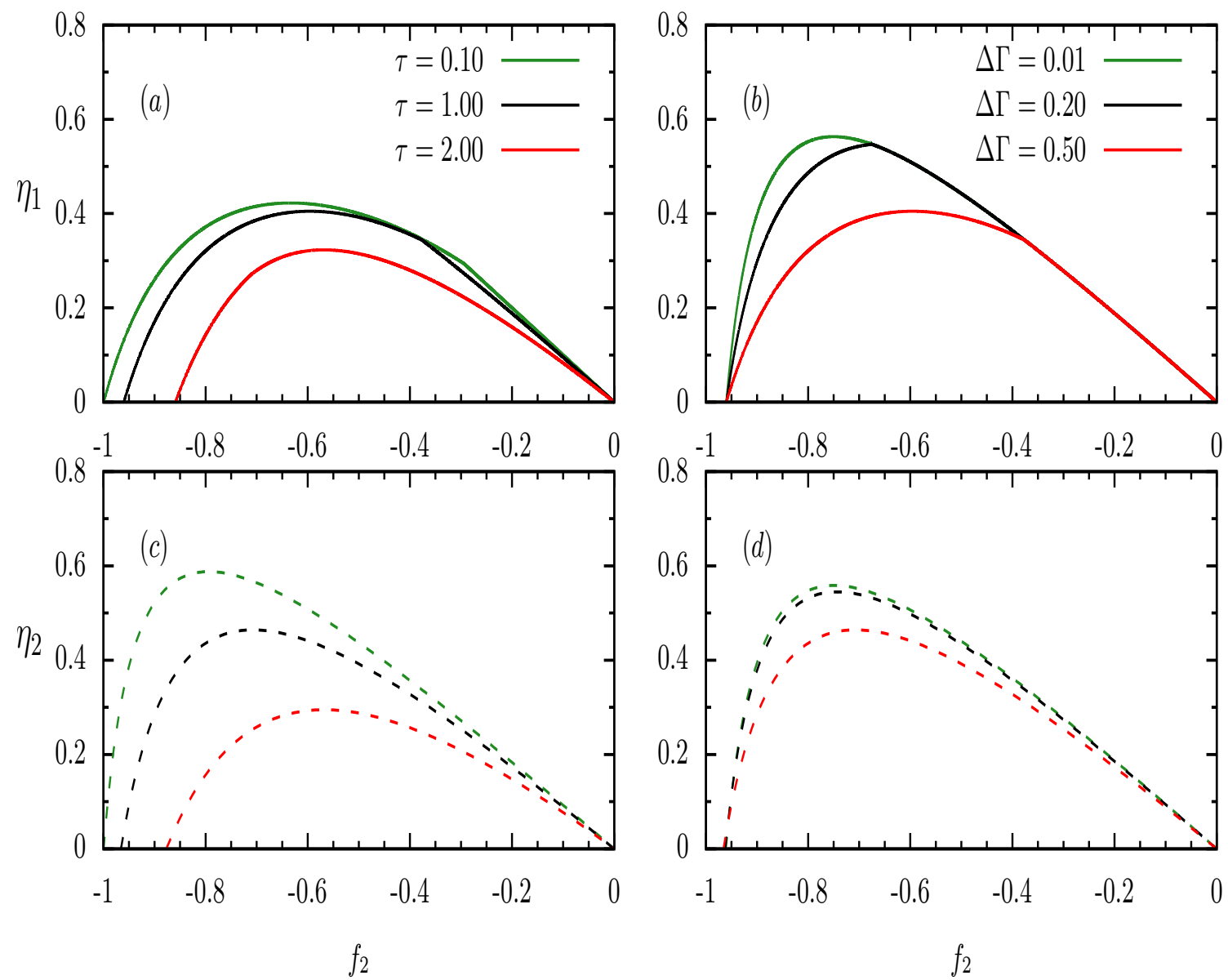

Figura 4.13: Painéis mostram a eficiências $\eta_{1,2}$ versus $f_{2}$. Painéis $(a)$ e $(c)$ são para $\Delta \Gamma=0.5$ e diferentes períodos $\tau^{\prime} s$. Painéis $(b)$ e $(d)$ são para $\tau=1.0$ e diferentes valores de $\Delta \Gamma^{\prime} s$. Subíndices em $\eta_{1,2}$ estão associados com Eqs.[4.18-4.19] respetivamente. 
CAPÍTULO 5

\section{Conclusões}

No trabalho apresentamos as propriedades termodinâmicas de uma partícula browniana colocada periodicamente em contato com reservatórios térmicos sequenciais. Estudamos as grandezas termodinâmicas do sistema e entre elas a eficiência, potência, produção de entropia, calor, de onde obtivemos expressões exatas para as mesmas.

Nossa descrição considera um número arbitrário de reservatórios sequenciais e a influência de forças externas. Considerações sobre a eficiência foram realizadas, nas quais as máquinas brownianas podem ser operadas adequadamente garantindo o compromisso confiável entre a eficiência e potência finita para pequenos períodos de estudo no sistema.

Como uma generalização de nosso trabalho introduzimos a ideia de tempos assimétricos, ou seja quando a partícula fica mais tempo em contato com um reservatório que com outro, estudando assim o papel da assimetria. Derivamos expressões gerais para as propriedades termodinâmicas considerando uma força constante e uma periódica (driving) qualquer.

Como comentário final, mencionamos as várias novas perspectivas a serem aborda- 
das, em primeiro lugar: estender esse estudo para outros protocolos de forças externas (por exemplo, os dependentes do tempo senoidais), a fim de comparar suas eficiências, principalmente com o caso de força linear e vincular as ideias gerais que foram estudadas a outros campos como : sistemas biofísicos, reações químicas e outros campos da ciência. Finalmente, seria muito notável verificar a validade das recentes propostas de relações de incertezas (TURs) para (FPE) em tal classe de sistemas [23],[24]. 
APÊNDICE $A$

Alguns cálculos analíticos.

\section{A.1 Equação de Fokker-Planck para o movimento brow- niano.}

Supondo que $P_{n}\left(v_{n}\right)$ é a distribuição de probabilidades da variável estocástica $v_{n}$ e $g_{n}(k)$ a função característica:

$$
g_{n}(k)=\left\langle e^{i k v_{n}}\right\rangle=\int e^{i k v_{n}} P_{n}\left(v_{n}\right) d v_{n}
$$

de forma que:

$$
g_{n+1}(k)=\left\langle e^{i k v_{n+1}}\right\rangle=\left\langle e^{i k\left[v_{n}+\tau f\left(v_{n}\right)+\tau \xi_{n}\right]}\right\rangle,
$$

onde tendo em conta a independência entre as variáveis $v_{n}$ e $\xi_{n}$ temos:

$$
g_{n+1}(k)=\left\langle e^{i k\left[v_{n}+\tau f\left(v_{n}\right)\right]}\right\rangle\left\langle e^{i k \tau \xi_{n}}\right\rangle .
$$

Fazendo uma expansão de $g_{n+1}(k)$ até primeira ordem em $\tau$ os termos do produto em 
Eq.[A.3], temos que:

$$
\left\langle e^{i k v_{n}}\left[1+i k \tau f\left(v_{n}\right)\right]\right\rangle=\left\langle e^{i k v_{n}}\right\rangle+i k \tau\left\langle f\left(v_{n}\right) e^{i k v_{n}}\right\rangle
$$

e:

$$
1+i k \tau\left\langle\xi_{n}\right\rangle-\frac{1}{2} k^{2} \tau^{2}\left\langle\xi_{n}^{2}\right\rangle=1-\frac{1}{2} k^{2} \tau \Gamma
$$

Usando as propriedades do ruído gaussiano $\left\langle\xi_{n}\right\rangle=0$ e $\left\langle\xi_{n}^{2}\right\rangle=\Gamma / \tau$ chegamos em:

$$
g_{n+1}(k)=g_{n}(k)+\tau\left[i k\left\langle f\left(v_{n}\right) e^{i k v_{n}}\right\rangle-\frac{\Gamma}{2} k^{2}\left\langle e^{i k v_{n}}\right\rangle\right]
$$

Asando as propriedades:

$$
i k\left\langle f(v) e^{i k v}\right\rangle=\left\langle f(v) \frac{d}{d v} e^{i k v}\right\rangle=-\int e^{i k v} \frac{d}{d v}\left[f(v) P_{n}(v)\right] d v,
$$

e:

$$
-k^{2}\left\langle e^{i k v}\right\rangle=\left\langle\frac{d^{2}}{d v^{2}} e^{i k v}\right\rangle=\int e^{i k v} \frac{d^{2}}{d v^{2}} P_{n}(v) d v
$$

chegamos a seguente expressão:

$$
P_{n+1}(v)-P_{n}(v)=-\tau \frac{d}{d v}\left[f(v) P_{n}(v)\right]+\tau \frac{\Gamma}{2} \frac{d^{2}}{d v^{2}} P_{n}(v)
$$

Multiplicando por $1 / \tau$ a expressão anterior e avaliando no limite $\tau \rightarrow \infty$ chegamos em:

$$
\frac{\partial}{\partial t} P(v, t)=-\frac{\partial}{\partial v}[f(v) P(v, t)]+\frac{\Gamma}{2} \frac{\partial^{2}}{\partial v^{2}} P(v, t)
$$

que é a equação de Fokker-Planck.

\section{A.2 Solução da equação de Fokker-Planck.}

Quando a partícula está em contato com um reservatório e a força que atua sob a particula tem a forma $f(v)=-\gamma v$, a equação de Fokker-Planck (FPE) neste caso fica da forma:

$$
\frac{\partial P}{\partial v}=\gamma \frac{\partial(v P)}{\partial v}-f \frac{\partial P}{\partial v}+\frac{\Gamma}{2} \frac{\partial^{2} P}{\partial v^{2}}
$$

onde $f=F(t) / m$ é a força por unidade de masa e $P=P(v, t)$ a distribuição de probabilidade, definindo agora a função característica $G(k, t)$ :

$$
G(k, t)=\int_{-\infty}^{\infty} P e^{i k v} d v
$$


Temos então que:

$$
\int_{-\infty}^{\infty} \frac{\partial P}{\partial v} e^{i k v} d v=\frac{\partial}{\partial v} \int_{-\infty}^{\infty} P e^{i k v} d v=\frac{\partial G}{\partial v}
$$

de forma que o primeiro termo do membro direito em Eq.[A.13] torna-se:

$$
\begin{gathered}
\gamma \int_{-\infty}^{\infty} \frac{\partial(v P)}{\partial v} e^{i k v} d v=-i \gamma k \int_{-\infty}^{\infty} v P e^{i k v} d v=-\gamma k \int_{-\infty}^{\infty} P \frac{\partial\left(e^{i k v}\right)}{\partial v} d v \\
=-\gamma k \frac{\partial}{\partial k} \int_{-\infty}^{\infty} P e^{i k v} d v=-\gamma k \frac{\partial G}{\partial k}
\end{gathered}
$$

onde usamos integração por partes e consideramos que $P(v, t)$ anula-se nas extremidades $(v \pm \infty)$. dessa forma, o segundo termo do membro direito de Eq.[A.13] torna-se:

$$
-f \int_{-\infty}^{\infty} \frac{\partial P}{\partial v} e^{i k v} d v=i k f \int_{-\infty}^{\infty} P e^{i k v} d v=-i k f G
$$

e finalmente temos:

$$
\frac{\Gamma}{2} \int_{-\infty}^{\infty} \frac{\partial^{2} P}{\partial v^{2}} e^{i k v} d v=-\frac{i k \Gamma}{2} \int_{-\infty}^{\infty} \frac{\partial P}{\partial v} e^{i k v} d v=-\frac{\Gamma k^{2} G}{2} .
$$

Assim usando as relações Eqs.[A.13-A.16] em Eq.[A.11] temos:

$$
\frac{\partial G}{\partial t}=-\gamma k \frac{\partial G}{\partial k}+i k f G-\frac{\Gamma k^{2} G}{2} .
$$

A equação diferencial acima posseei solução dada por:

$$
G(k, t)=\exp \left\{i a(t) k-\frac{b(t) k^{2}}{2}\right\},
$$

onde:

$$
a(t)=C e^{-\gamma t}+\frac{f}{\gamma} \quad, \quad b(t)=\frac{\Gamma}{2 \gamma}+A e^{-2 \gamma t} .
$$

Conforme mostramos anteriormente, $a(t)$ é a velocidade média da partícula browniana e $b(t)$ sua variância. 


\section{A.3 Produção de Entropia.}

Consideremos uma a solução da equação diferençal Eq.[A.18] para $k$-reservatórios arbitrários:

$$
b_{k}(t)=\frac{\Gamma_{k}}{2 \gamma}+A_{k} e^{-2 \gamma\left(t-\tau_{k-1}\right)},
$$

onde $\tau_{k}=k \tau / N$ e $k=1,2,3, \ldots, N$, assim aplicando a continuidade da variância temos:

$$
b_{k}\left(\tau_{k}\right)=b_{k+1}\left(\tau_{k}\right) ; \quad \forall k=1,2,3, \ldots, N-1,
$$

então como consequência de Eq.[A.21] temos:

$$
A_{k+1}=x A_{k}+\frac{1}{2 \gamma}\left(\Gamma_{k}-\Gamma_{k+1}\right),
$$

onde $x=e^{-2 \gamma \tau / N}$, isso representa uma recorrência linear não homogênea que pode ser resolvida por substituição:

$$
A_{k}=x^{k-1} A_{1}+\frac{1}{2 \gamma} \sum_{l=2}^{k} x^{k-l}\left(\Gamma_{l-1}-\Gamma_{l}\right) ; \quad \forall k=2,3, \ldots, N
$$

Para demonstrar que Eq.[A.23] representa a solução de Eq.[A.22] usaremos o primeiro princípio de indução completa .

Por outro lado, usando as condições de contorno periódicas, temos que:

$$
b_{N}(\tau)=b_{1}(0) \Rightarrow A_{N}+x^{-1} A_{1}+\frac{x^{-1}}{2 \gamma}\left(\Gamma_{1}-\Gamma_{N}\right)
$$

assim usando as relações Eq.[A.23],[A.24] para o caso $k=N$ temos:

$$
A_{1}=\frac{1}{2 \gamma} \frac{x^{N}}{1-x^{N}} \sum_{i=1}^{N} x^{-l}\left(\Gamma_{l}-\Gamma_{l+1}\right),
$$

então assim a constante $A_{k}$ é dada por:

$$
A_{k}=\frac{1}{2 \gamma} \frac{x^{k-1}}{1-x^{N}}\left[\sum_{l=1}^{k-1} x^{-l}\left(\Gamma_{l}-\Gamma_{l+1}\right)+\sum_{l=k}^{N}\left(\Gamma_{l}-\Gamma_{l+1}\right)\right]
$$


A partir dos coeficientes acima, o fluxo de entropia nos intervalo $(k-1) \tau / N \gtrless t \gtrless$ $k \tau / N$ é dado por:

$$
\Phi_{k}(t)=\frac{2 \gamma^{2}}{\Gamma_{k}} b_{k}(t)-\gamma=\frac{2 \gamma}{\Gamma_{k}} A_{k} e^{-2 \gamma\left(t-\tau_{k-1}\right)},
$$

portanto a produção de entropia calculada sobre um período é a soma das contribuições a cada intervalo de tempo, dada por:

$$
\begin{gathered}
\bar{\Pi}=\frac{1}{\tau} \sum_{k=1}^{N} \int_{\tau_{k-1}}^{\tau_{k}} \Phi(t) d t=\frac{2 \gamma}{\tau} \sum_{k=1}^{N} \frac{A_{k}}{\Gamma_{k}} \int_{\tau_{k-1}}^{\tau_{k}} e^{-2 \gamma\left(t-\tau_{k-1}\right)} d t \\
=\frac{\gamma}{\tau} \sum_{k=1}^{N} \frac{A_{k}}{\Gamma_{k}} \int_{0}^{2 \gamma \tau / N} e^{-t} d t=\frac{\gamma}{\tau}(1-x) \sum_{k=1}^{N} \frac{A_{k}}{\Gamma_{k}},
\end{gathered}
$$

usando Eqs.[A.25-A.26] temos:

$$
\begin{aligned}
2 \gamma \sum_{k=1}^{N} \frac{A_{k}}{\Gamma_{k}}= & \frac{x^{N-1}}{1-x^{N}} \sum_{k=1}^{N} \frac{\Gamma_{k}-\Gamma_{k+1}}{\Gamma_{k}}+\frac{x^{N-2}}{1-x^{N}} \sum_{k=1}^{N} \frac{\Gamma_{k+1}-\Gamma_{k+2}}{\Gamma_{k}}+ \\
& +\ldots+\frac{x^{N-l}}{1-x^{N}} \sum_{k=1}^{N} \frac{\Gamma_{k+l-1}-\Gamma_{k+l}}{\Gamma_{k}}+\ldots+ \\
& +\frac{1}{1-x^{N}} \frac{\Gamma_{k+N-1}-\Gamma_{k+N}}{\Gamma_{k}}
\end{aligned}
$$

ou:

$$
2 \gamma \sum_{k=1}^{N} \frac{A_{k}}{\Gamma_{k}}=\frac{x^{N}}{1-x^{N}} \sum_{k, l=1}^{N} x^{-l}\left(\frac{\Gamma_{k+l-1}-\Gamma_{k+l}}{\Gamma_{k}}\right) .
$$

Dessa forma, reescrevendo Eq.[A.28] temos:

$$
\bar{\Pi}=\frac{x^{N}(1-x)}{1-x^{N}} \sum_{k, l=1}^{N} x^{-l}\left(\frac{\Gamma_{k+l-1}-\Gamma_{k+l}}{\Gamma_{k}}\right),
$$

onde usando as condições de contorno dadas pela Eq.[A.31] temos:

$$
\bar{\Pi}=-\frac{N}{2 \tau}\left(\frac{1-x}{x}\right)+\frac{1}{2 \tau} \frac{x^{N-1}(1-x)^{2}}{1-x^{N}} \sum_{k, l=1}^{N} x^{-l} \frac{\Gamma_{k+l}}{\Gamma_{k}},
$$

usando a desigualdade de Cauchy, temos que:

$$
\frac{1}{N} \sum_{k=1}^{N} \frac{\Gamma_{k+l}}{\Gamma_{k}} \geq \sqrt[N]{\prod_{k=1}^{N} \frac{\Gamma_{k+l}}{\Gamma_{k}}}=1 \Rightarrow \sum_{k=1}^{N} \frac{\Gamma_{k+l}}{\Gamma_{k}} \geq N
$$


portanto:

$$
\sum_{k, l=1}^{N} \frac{\Gamma_{k+l}}{\Gamma_{k}} \geq N \sum_{l=1}^{N} x^{-l}=\frac{N}{x^{N}}\left(\frac{1-x^{N}}{1-x}\right)
$$

e finalmente:

$$
\bar{\Pi} \geq \frac{-N}{2 \tau}\left(\frac{1-x}{x}\right)+\frac{N}{2 \tau}\left(\frac{1-x}{x}\right)=0,
$$

em consistência com a primeira lei da termodinâmica. 


\section{A.4 Produção de entropia para o caso de força constante simétrico.}

Partindo da solução de Eq.[3.10] para o caso de força constante temos:

$$
\left\langle v_{i}\right\rangle(t)=e^{-\gamma_{i}\left(t-\tau_{i-1}\right)}\left[A_{i-1}+\int_{\tau_{i-1}}^{t} e^{\gamma_{i}\left(t^{\prime}-\tau_{i-1}\right)} f_{i}\left(t^{\prime}\right) d t^{\prime}\right] .
$$

Para o caso simétrico, ou assimétrico, as condições de contorno da velocidade média são dadas por:

$$
\left\langle v_{2}\right\rangle(\tau)=\left\langle v_{1}\right\rangle(0) \quad, \quad\left\langle v_{2}\right\rangle\left(\tau_{1}\right)=\left\langle v_{1}\right\rangle\left(\tau_{1}\right)
$$

Uma vez que a variância é somente função da força, sua expressão e mais simples e dada por:

$$
b_{1}(t)=A_{1} e^{-2 \gamma t}+\frac{\Gamma_{1}}{2 \gamma} \quad, \quad b_{2}(t)=A_{2} e^{-2 \gamma\left(t-\tau_{1}\right)}+\frac{\Gamma_{2}}{2 \gamma},
$$

usando as condições de continuidade:

$$
b_{1}(0)=b_{2}(\tau) \quad, \quad b_{1}\left(\tau_{1}\right)=b_{2}\left(\tau_{1}\right),
$$

substituindo Eq.[A.39] em Eq.[A.38] temos o seguente sistema de equações:

$$
A_{1} e^{-2 \gamma(0)}+\frac{\Gamma_{1}}{2 \gamma}=A_{2} e^{-2 \gamma\left(\tau-\tau_{1}\right)}+\frac{\Gamma_{2}}{2 \gamma}
$$

$\mathrm{e}$

$$
A_{1} e^{-2 \gamma\left(\tau_{1}\right)}+\frac{\Gamma_{1}}{2 \gamma}=A_{2} e^{-2 \gamma(0)}+\frac{\Gamma_{2}}{2 \gamma}
$$

Resolvendo agora o sistema Eqs.[A.40-A.41] para $A_{1}$ temos:

$$
A_{1}=-\frac{\left(\Gamma_{1}-\Gamma_{2}\right)\left(e^{2 \gamma \tau_{1}}-e^{2 \gamma \tau}\right)}{2 \gamma\left(e^{2 \gamma \tau}-1\right)},
$$

introduzindo a variável $\kappa=\tau_{1} / \tau_{2}$, temos que:

$$
\kappa=\frac{\tau_{1}}{\tau_{2}} \quad, \quad \tau_{1}+\tau_{2}=\tau \Rightarrow \quad \tau_{1}=\frac{\kappa \tau}{1+\kappa} \quad \text { e } \quad \tau_{2}=\frac{\tau}{1+\kappa},
$$

de forma que Eq.[A.42] torna-se:

$$
A_{1}=-\frac{\left(\Gamma_{1}-\Gamma_{2}\right)\left(1-e^{-\frac{2 \gamma \tau}{1+\kappa}}\right)}{2 \gamma\left(1-e^{-2 \gamma \tau}\right)}
$$


Realizando o mesmo procedimento para a constante $A_{2}$ temos:

$$
A_{2}=\frac{\left(\Gamma_{1}-\Gamma_{2}\right)\left(1-e^{-\frac{2 \gamma \kappa \tau}{1+\kappa}}\right)}{2 \gamma\left(1-e^{-2 \gamma \tau}\right)}
$$

e com mudança de variável Eq.[A.43] em Eq.[A.38 a variância é dada por:

$$
b_{1}(t)=-\frac{\left(\Gamma_{1}-\Gamma_{2}\right)\left(1-e^{-\frac{2 \gamma \tau}{1+\kappa}}\right)}{2 \gamma\left(1-e^{-2 \gamma \tau}\right)} e^{-2 \gamma t}+\frac{\Gamma_{1}}{2 \gamma}
$$

e

$$
b_{2}(t)=\frac{\left(\Gamma_{1}-\Gamma_{2}\right)\left(1-e^{-\frac{2 \gamma \kappa \tau}{1+\kappa}}\right)}{2 \gamma\left(1-e^{-2 \gamma \tau}\right)} e^{-2 \gamma\left(t-\frac{\kappa \tau}{1+\kappa}\right)}+\frac{\Gamma_{2}}{2 \gamma} .
$$

Substituindo a velocidade média ao quadrado da solução de Eq.[A.36], temos as seguentes expressões:

$$
\left\langle v^{2}\right\rangle_{1,2}(t)=b_{1,2}(t)+\langle v\rangle_{1,2}^{2}(t)
$$

e portanto:

$$
\left\langle v_{1}^{2}\right\rangle(t)=\frac{\Gamma_{1}}{2 \gamma}-\frac{\left(\Gamma_{1}-\Gamma_{2}\right)\left(e^{-\frac{2 \gamma \tau}{1+\kappa}}-1\right) e^{-2 \gamma\left(t-\frac{\gamma \kappa \tau}{1+\kappa}\right)}}{2 \gamma\left(e^{2 \gamma \tau}-1\right)}
$$

$\mathrm{e}$

$$
\left\langle v_{2}^{2}\right\rangle(t)=\frac{\Gamma_{2}}{2 \gamma}+\frac{\left(\Gamma_{1}-\Gamma_{2}\right)\left(e^{\frac{2 \gamma \tau}{1+\kappa}}-1\right) e^{-2 \gamma(t-\tau)}}{2 \gamma\left(e^{2 \gamma \tau}-1\right)} .
$$

Para o cálculo dos fluxos temos:

$$
\phi_{1}(t)=\frac{2 \gamma^{2}}{\Gamma_{1}}\left\langle v_{1}^{2}\right\rangle(t)-\gamma \quad, \quad \phi_{2}(t)=\frac{2 \gamma^{2}}{\Gamma_{2}}\left\langle v_{2}^{2}\right\rangle(t)-\gamma,
$$

substituindo assim Eqs.[A.49],[A.50] em Eq.[A.51] respetivamente, temos o fluxo associado à diferencia de temperaturas é dada por:

$$
\phi_{1}(t)=-\frac{\gamma\left(\Gamma_{1}-\Gamma_{2}\right)\left(e^{\frac{2 \gamma \tau}{1+\kappa}}-1\right) e^{2 \gamma\left(\frac{\kappa \tau}{1+\kappa}-t\right)}[\operatorname{coth}(\gamma \tau)-1]}{2 \Gamma_{1}} \quad, \quad 0<t \leq \frac{\kappa \tau}{1+\kappa}
$$

$\mathrm{e}$

$$
\phi_{2}(t)=\frac{\gamma\left(\Gamma_{1}-\Gamma_{2}\right)\left(e^{\frac{2 \gamma \kappa \tau}{1+\kappa}}-1\right) e^{2 \gamma(\tau-t)}[\operatorname{coth}(\gamma \tau)-1]}{2 \Gamma_{2}} \quad, \quad \frac{\kappa \tau}{1+\kappa}<t \leq \tau
$$

Definindo a produção de entropia média da seguente forma:

$$
\Pi=\frac{1}{\tau}\left[\int_{0}^{\frac{\kappa \tau}{1+\kappa}} \Phi_{1}(t) d t+\int_{\frac{\kappa \tau}{1+\kappa}}^{\tau} \Phi_{2}(t) d t\right]
$$


temos, substituindo Eqs.[A.52,A.53] em Eq.[A.54] que:

$$
\Pi=\frac{\Gamma_{1} \Gamma_{2}}{\tau} \frac{\sinh \left[\frac{\gamma \kappa \tau}{1+\kappa}\right] \sinh \left[\frac{\gamma \tau}{1+\kappa}\right]}{\sinh [\gamma \tau]}\left(\frac{1}{\Gamma_{1}}-\frac{1}{\Gamma_{2}}\right)^{2} .
$$

Notemos que para o caso $\kappa=1$, temos a identidade:

$$
\frac{\sinh \left[\frac{\gamma \tau}{2}\right] \sinh \left[\frac{\gamma \tau}{2}\right]}{\sinh [\gamma \tau]}=\frac{1}{2} \tanh \left[\frac{\gamma \tau}{2}\right]
$$

portanto, para o caso simétrico a produção de entropia tem a forma:

$$
\Pi=\frac{\Gamma_{1} \Gamma_{2}}{2 \tau} \tanh \left[\frac{\gamma \tau}{2}\right]\left(\frac{1}{\Gamma_{1}}-\frac{1}{\Gamma_{2}}\right)^{2} .
$$

Para o caso da força termodinâmica dependente da temperatura $F_{T}=\left(\frac{1}{\Gamma_{1}}-\frac{1}{\Gamma_{2}}\right)$. Para $\Gamma_{1} \approx \Gamma_{2}$, podemos escrever a produção de entropia na forma $\Pi=L_{T T} F_{T}^{2}$, assim o coeficiente de Onsager $L_{T T}$ fica:

$$
L_{T T}=\frac{\Gamma_{1}^{2}}{\tau} \frac{\sinh \left[\frac{\gamma \kappa \tau}{1+\kappa}\right] \sinh \left[\frac{\gamma \tau}{1+\kappa}\right]}{\sinh [\gamma \tau]}
$$

No caso de força linear é exatamente a mesma metodologia para obter a produção de entropia.

\section{A.5 Artigo publicado}

Nesta seção serão apresentados os principais resultados relacionados com esta dissertação de mestrado. 


\title{
Thermodynamics of collisional models for Brownian particles: General properties and efficiency
}

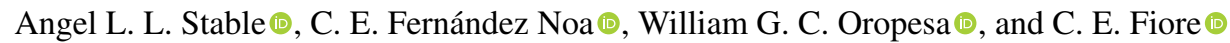 \\ Universidade de São Paulo, Instituto de Física, Rua do Matão, 1371, 05508-090 São Paulo, São Paulo, Brasil
}

(Received 1 May 2020; accepted 2 September 2020; published 2 October 2020)

\begin{abstract}
We introduce the idea of collisional models for Brownian particles, in which a particle is sequentially placed in contact with distinct thermal environments and external forces. Thermodynamic properties are exactly obtained, irrespective of the number of reservoirs involved. In the presence of external forces, the entropy production presents a bilinear form in which Onsager coefficients are exactly calculated. Analysis of Brownian engines based on sequential thermal switchings is proposed and considerations about their efficiencies are investigated, taking into account distinct external forces protocols. Our results shed light to an alternative route for obtaining efficient thermal engines based on finite times Brownian machines.
\end{abstract}

DOI: 10.1103/PhysRevResearch.2.043016

\section{INTRODUCTION}

Stochastic thermodynamics has proposed a general and unified scheme for addressing central issues in thermodynamics [1-5]. It includes not only an extension of concepts from equilibrium to nonequilibrium systems but also it deals with the existence of new definitions and bounds [6-9], general considerations about the efficiency of engines at finite time operations [1-3], and others aspects. In all cases, the concept of entropy production $[1,4,10]$ plays a central role, being a quantity continuously produced in nonequilibrium steady states (NESS), whose main properties and features have been extensively studied in the last years, including its usage for typifying phase transitions [11-14].

Basically, a NESS can be generated under two fundamental ways: From fixed thermodynamic forces $[15,16]$ or from time-periodic variation of external parameters [17-20]. In this contribution, we address a different kind of periodic driving, suitable for the description of engineered reservoirs, at which a system interacts sequentially and repeatedly with distinct environments [21-23]. Commonly referred as collisional models, they have been inspired by the assumption that in many cases (e.g., the original Brownian motion) a particle collides only with few molecules of the environment and then the subsequent collision will occur with another fraction of uncorrelated molecules. Collisional models have been viewed as more realistic frameworks in certain cases, encompassing not only particles interacting with a small fraction of the environment but also those presenting distinct drivings over each member of system [24-27] or even species yielding a weak coupling with the reservoir. More recently, they have been (broadly) extended for quantum systems for mimicking the environment, represented by a weak interaction between the

Published by the American Physical Society under the terms of the Creative Commons Attribution 4.0 International license. Further distribution of this work must maintain attribution to the author $(s)$ and the published article's title, journal citation, and DOI. system and a sequential collection of uncorrelated particles [28-30].

With the above in mind, we introduce the concept of repeated interactions for Brownian particles. More specifically, a particle under the influence of a given external force is placed in contact with a reservoir during the time interval and afterwards it is replaced by an entirely different (and independent) set of interactions. Exact expressions for thermodynamic properties are derived and the entropy production presents a bilinear form, in which Onsager coefficients are obtained as function of period. Considerations about the efficiency are undertaken and a suited regime for the system operating as an efficient thermal machine is investigated.

The present study sheds light for fresh perspectives in nonequilibrium thermodynamics, including the possibility of experimental buildings of heat engines based on Brownian dynamics [31-36] with sequential reservoirs. Also, they provide us the extension and validation of recent bounds between currents and entropy production, the so called thermodynamic uncertainty relations (TURs) [8,9,37-41], which has aroused a recent and great interest.

This paper is organized as follows: Secs. II and III present the model description and its exact thermodynamic properties. In Sec. IV we extend analysis for external forces and considerations about efficiency are performed in Sec. V. Conclusions and perspectives are drawn in Sec. VI.

\section{MODEL AND FOKKER-PLANCK EQUATION}

We are dealing with a Brownian particle with mass $m$ sequentially placed in contact with $N$ different thermal reservoirs. Each contact has a duration of $\tau / N$ and occurs during the intervals $\tau_{i-1} \leqslant t<\tau_{i}$, where $\tau_{i}=i \tau / N$ for $i=1, . ., N$, in which the particle evolves in time according to the following Langevin equation:

$$
m \frac{d v_{i}}{d t}=-\alpha_{i} v_{i}+F_{i}(t)+B_{i}(t)
$$

where quantities $v_{i}, \alpha_{i}$, and $F_{i}(t)$ denote the particle velocity, the viscous constant and external force, respectively. From 
now on, we shall express them in terms of reduced quantities: $\gamma_{i}=\alpha_{i} / m$ and $f_{i}(t)=F_{i}(t) / m$. The stochastic force $\zeta_{i}(t)=$ $B_{i}(t) / m$ accounts for the interaction between particle and the $i$ th environment and satisfies the properties

$$
\left\langle\zeta_{i}(t)\right\rangle=0
$$

and

$$
\left\langle\zeta_{i}(t) \zeta_{i^{\prime}}\left(t^{\prime}\right)\right\rangle=2 \gamma_{i} T_{i} \delta_{i i^{\prime}} \delta\left(t-t^{\prime}\right),
$$

respectively, where $T_{i}$ is the bath temperature. Let $P_{i}(v, t)$ be the velocity probability distribution at time $t$, its time evolution is described by the Fokker-Planck (FP) equation $[3,16,42]$

$$
\frac{\partial P_{i}}{\partial t}=-\frac{\partial J_{i}}{\partial v}-f_{i}(t) \frac{\partial P_{i}}{\partial v},
$$

where $J_{i}$ is given by

$$
J_{i}=-\gamma_{i} v P_{i}-\frac{\gamma_{i} k_{\mathrm{B}} T_{i}}{m} \frac{\partial P_{i}}{\partial v} .
$$

It is worth mentioning that above equations are formally identical to description of the overdamped harmonic oscillator subject to the harmonic force $f_{h}=-\bar{k} x$ just by replacing $x \rightarrow v, \bar{k} / \alpha \rightarrow \gamma_{i}, 1 / \alpha \rightarrow \gamma_{i} / m$.

From the FP equation and by performing appropriate partial integrations together boundary conditions in which both $P_{i}(v, t)$ and $J_{i}(v, t)$ vanish at extremities, the time variation of the energy system $U_{i}=\left\langle E_{i}\right\rangle$ in contact with the $i$ th reservoir is given by

$$
\frac{d U_{i}}{d t}=-\frac{m}{2} \int v^{2}\left[\frac{\partial J_{i}}{\partial v}+f_{i}(t) \frac{\partial P_{i}}{\partial v}\right] d v .
$$

The right side of Eq. (6) can be rewritten as $d U_{i} / d t=-\left(\dot{W}_{i}+\right.$ $\dot{Q}_{i}$ ), where $\dot{W}_{i}$ and $\dot{Q}_{i}$ denote the work per unity of time and heat flux from the system to the environment (thermal bath) given by

$$
\dot{W}_{i}=-m\left\langle v_{i}\right\rangle f_{i}(t) \quad \text { and } \quad \dot{Q}_{i}=\gamma_{i}\left(m\left\langle v_{i}^{2}\right\rangle-k_{\mathrm{B}} T_{i}\right),
$$

respectively. In the absence of external forces $\dot{W}_{i}=0$ and all heat flux comes from/goes to the thermal bath.

By assuming the system entropy $S$ is given by $S_{i}(t)=$ $-k_{\mathrm{B}} \int P_{i}(v, t) \ln \left[P_{i}(v, t)\right] d v$ and from the expression for $J_{i}$, one finds that its time derivative is given by

$$
\frac{d S_{i}}{d t}=-k_{\mathrm{B}} \int\left(\frac{J_{i}}{P_{i}}\right)\left(\frac{\partial P_{i}}{\partial v}\right) d v .
$$

As for the mean energy, above expression can be rewritten in the following form:

$$
\frac{d S_{i}}{d t}=\frac{m}{\gamma_{i} T_{i}}\left(\int \frac{J_{i}^{2}}{P_{i}} d v+\gamma_{i} \int v J_{i} d v\right) .
$$

Equation (9) can be interpreted according to the following form: $d S_{i} / d t=\Pi_{i}(t)-\Phi_{i}(t)[16,42]$, where the former term corresponds to the entropy production rate $\Pi_{i}(t)$ and it is strictly positive (as expected). The second term is the the flux of entropy and can also be rewritten more conveniently as

$$
\Phi_{i}(t)=\frac{\dot{Q}_{i}}{T_{i}}=\gamma_{i}\left(\frac{m}{T_{i}}\left\langle v_{i}^{2}\right\rangle-k_{\mathrm{B}}\right) .
$$

If external forces are null and the particle is placed in contact to a single reservoir, then the probability distribution approaches for large times the Gibbs (equilibrium) distribution $P_{i}^{\mathrm{eq}}(v)=e^{-E / k_{\mathrm{B}} T_{i}} / Z$, with $E=m v^{2} / 2$ its kinetic energy and $Z$ the partition function. In such case, $\left\langle v_{i}^{2}\right\rangle=k_{\mathrm{B}} T_{i} / m$ and therefore $\Pi_{\mathrm{eq}}=\Phi_{\mathrm{eq}}=0$ (as expected). Conversely, it will evolve to a nonequilibrium steady state (NESS) when placed in contact with sequential and distinct reservoirs, in which heat is dissipated and the entropy is produced and hence $\Pi_{\mathrm{NESS}}=\Phi_{\mathrm{NESS}}>0$.

\section{EXACT SOLUTION FOR ARBITRARY SET OF SEQUENTIAL RESERVOIRS}

From now on, quantities will be expressed in terms of the "reduced temperature" $\Gamma_{i}=2 \gamma_{i} k_{\mathrm{B}} T_{i} / m$ and $k_{B}=1$. Since we are dealing with a linear force on the velocity, the NESS will also be characterized by a Gaussian probability distribution $P_{i}(v, t)=e^{-\left(v-\left\langle v_{i}\right\rangle\right)^{2} / 2 b_{i}(t)} / \sqrt{2 \pi b_{i}(t)}$ in which both mean $\left\langle v_{i}\right\rangle(t)$ and the variance $b_{i}(t) \equiv\left\langle v_{i}^{2}\right\rangle(t)-\left\langle v_{i}\right\rangle^{2}(t)$ will be in general time-dependent. Their expressions can be calculated from Eqs. (4) and (5) and read

$$
\frac{d}{d t}\left\langle v_{i}\right\rangle=-\gamma_{i}\left\langle v_{i}\right\rangle+f_{i}(t)
$$

and

$$
\frac{d}{d t} b_{i}(t)=-2 \gamma_{i} b_{i}(t)+\Gamma_{i}
$$

respectively, where appropriate partial integrations were performed. Their solutions are given by the following expressions:

$$
\left\langle v_{i}\right\rangle(t)=e^{-\gamma_{i}\left(t-\tau_{i-1}\right)}\left[v_{i-1}^{\prime}+\int_{\tau_{i-1}}^{t} e^{\gamma_{i}\left(t^{\prime}-\tau_{i-1}\right)} f_{i}\left(t^{\prime}\right) d t^{\prime}\right]
$$

and

$$
b_{i}(t)=A_{i-1} e^{-2 \gamma_{i}\left(t-\tau_{i-1}\right)}+\frac{\Gamma_{i}}{2 \gamma_{i}}
$$

respectively, where quantities $v_{i-1}^{\prime} \equiv\left\langle v_{i}\right\rangle\left(\tau_{i-1}\right)$ and $A_{i}$ 's are evaluated by taking into account the set of continuity relations for the averages and variances, $\left\langle v_{i}\right\rangle\left(\tau_{i}\right)=\left\langle v_{i+1}\right\rangle\left(\tau_{i}\right)$ and $b_{i}\left(\tau_{i}\right)=b_{i+1}\left(\tau_{i}\right)$ (for all $\left.i=1, \ldots, N\right)$, respectively. Since the system returns to the initial state after a complete period, $\left\langle v_{1}\right\rangle(0)=\left\langle v_{N}\right\rangle(\tau)$ and $b_{1}(0)=b_{N}(\tau)$, all coefficients can be solely calculated in terms of model parameters, temperature reservoirs and the period. Also, the above conditions state that the probability at each point returns to the same value after every period.

For simplicity, from now on we shall assume the same viscous constant $\gamma_{i}=\gamma$ for all $i$ 's. In the absence of external forces, all $v_{i}^{\prime}$ 's vanish and the entropy production only depends on the coefficients $A_{i}$ 's and $\Gamma_{i}$ 's. Hence, the coefficient $A_{i}$ becomes

$$
A_{i+1}=x A_{i}+\frac{1}{2 \gamma}\left(\Gamma_{i}-\Gamma_{i+1}\right)
$$


where $x=e^{-2 \gamma \tau / N}$ and all of them can be found from a linear recurrence relation

$$
A_{i}=x^{i-1} A_{1}+\frac{1}{2 \gamma} \sum_{l=2}^{i} x^{i-l}\left(\Gamma_{l-1}-\Gamma_{l}\right)
$$

for $i=2, \ldots . N$. As the particle returns to the initial configuration after a complete period, $A_{N}$ then reads

$$
A_{N}=x^{-1} A_{1}+\frac{x^{-1}}{2 \gamma}\left(\Gamma_{1}-\Gamma_{N}\right) .
$$

By equaling Eqs. (16) and (17) for $i=N$, all coefficients $A_{i}$ 's can be finally calculated and are given by

$$
A_{1}=\frac{1}{2 \gamma} \frac{x^{N}}{1-x^{N}} \sum_{l=1}^{N} x^{-l}\left(\Gamma_{l}-\Gamma_{l+1}\right)
$$

and

$A_{i}=\frac{1}{2 \gamma} \frac{x^{i-1}}{1-x^{N}}\left[\sum_{l=1}^{i-1} x^{-l}\left(\Gamma_{l}-\Gamma_{l+1}\right)+\sum_{l=i}^{N} x^{N-l}\left(\Gamma_{l}-\Gamma_{l+1}\right)\right]$,

for $i=1$ and $i>1$, respectively. As we are focusing on the steady-state time-periodic regime, thermodynamic quantities can be averaged over one period $\tau$. The mean entropy production $\bar{\Pi}$ then reads

$$
\bar{\Pi}=\frac{1}{\tau} \sum_{i=1}^{N} \int_{\tau_{i-1}}^{\tau_{i}} \Phi_{i}(t) d t=\frac{\left(1-e^{-2 \gamma \tau / N}\right)}{2 \gamma \tau} \sum_{i=1}^{N} \frac{A_{i}}{\Gamma_{i}} .
$$

From Eqs. (18) and (19), it follows that

$$
\sum_{i=1}^{N} \frac{A_{i}}{\Gamma_{i}}=\frac{x^{N}}{1-x^{N}} \sum_{i, l=1}^{N} x^{-l}\left(\frac{\Gamma_{i+l-1}-\Gamma_{i+l}}{\Gamma_{i}}\right),
$$

and we arrive at an expression for $\bar{\Pi}$ solely dependent on the model parameters

$$
\bar{\Pi}=-\frac{N}{2 \gamma \tau}\left(\frac{1-x}{x}\right)+\frac{1}{2 \gamma \tau} \cdot \frac{x^{N-1}(1-x)^{2}}{1-x^{N}} \sum_{i, l=1}^{N} x^{-l} \frac{\Gamma_{i+l}}{\Gamma_{i}} .
$$

To show that $\bar{\Pi} \geqslant 0$, we resort to the inequality $\sum_{i=1}^{N} \Gamma_{i+l} / \Gamma_{i} \geqslant N \sqrt[N]{\prod_{i=1}^{N} \Gamma_{i+l} / \Gamma_{i}}$ for showing that $\sum_{i=1}^{N} \Gamma_{i+l} / \Gamma_{i} \geqslant N$, and hence Eq. (22) fulfills the condition

$$
\bar{\Pi} \geqslant-\frac{N}{2 \gamma \tau}\left(\frac{1-x}{x}\right)+\frac{N}{2 \gamma \tau}\left(\frac{1-x}{x}\right)=0,
$$

in consistency with the second law of thermodynamics.

As an concrete example, we derive explicit results for the two sequential reservoirs case. From Eqs. (13) and (14), coefficients $A_{1}$ and $A_{2}$ reduce to the following expressions:

$$
A_{1}=\frac{\Gamma_{2}-\Gamma_{1}}{2 \gamma}\left(\frac{1-e^{-\gamma \tau}}{1-e^{-2 \gamma \tau}}\right)=\frac{\Gamma_{2}-\Gamma_{1}}{2 \gamma}\left(\frac{1}{1+e^{\gamma \tau}}\right),
$$

where $A_{2}=-A_{1}$ and hence

$$
\Phi_{1}(t)=\gamma\left(\frac{\Gamma_{2}-\Gamma_{1}}{\Gamma_{1}}\right)\left(\frac{1}{1+e^{2 \gamma \tau}}\right) e^{-2 \gamma t},
$$

for $0 \leqslant t<\tau / 2$ and

$$
\Phi_{2}(t)=\gamma\left(\frac{\Gamma_{1}-\Gamma_{2}}{\Gamma_{2}}\right)\left(\frac{1}{1+e^{2 \gamma \tau}}\right) e^{-2 \gamma\left(t-\frac{\tau}{2}\right)},
$$

$\tau / 2 \leqslant t<\tau$, respectively, whose average entropy production reads

$$
\bar{\Pi}=\left[\frac{\Gamma_{1} \Gamma_{2}}{2 \tau} \tanh \left(\frac{\gamma \tau}{2}\right)\right]\left(\frac{1}{\Gamma_{1}}-\frac{1}{\Gamma_{2}}\right)^{2} .
$$

Note that $\bar{\Pi} \geqslant 0$ and it vanishes when $\Gamma_{1}=\Gamma_{2}$. In the limit of slow $(\tau \gg 1)$ and fast $(\tau<<1)$ oscillations, $\bar{\Pi}$ approaches to the following asymptotic expressions:

$$
\bar{\Pi} \approx \frac{\Gamma_{1} \Gamma_{2}}{2 \tau}\left(\frac{1}{\Gamma_{1}}-\frac{1}{\Gamma_{2}}\right)^{2} \text { and } \frac{\Gamma_{1} \Gamma_{2} \gamma}{4}\left(\frac{1}{\Gamma_{1}}-\frac{1}{\Gamma_{2}}\right)^{2},
$$

respectively, and such a latter expression is independent on the period.

Equation (27) can be conveniently written down as a flux-times-force expression, where the thermodynamic force attempts to the difference of temperatures of reservoirs. Given that the viscous coefficient is the same for all switchings, the thermodynamic force can be more conveniently expressed in terms of difference of $\Gamma_{i}$ 's. More specifically, we have that $\bar{\Pi}=\mathcal{J}_{T} f_{T}$, where $f_{T}=\left(1 / \Gamma_{2}-1 / \Gamma_{1}\right)$ and $\mathcal{J}_{T}$ can also be rewritten as $\mathcal{J}_{T}=L_{T T} f_{T}$, where $L_{T T}$ is the Onsager coefficient given by

$$
L_{T T}=\frac{\Gamma_{1} \Gamma_{2}}{2 \tau} \tanh \left(\frac{\gamma \tau}{2}\right) .
$$

Note that $L_{T T} \geqslant 0$ (as expected).

Figure 1 depicts the average entropy production $\bar{\Pi}$ versus $\tau$ for distinct values of $\Gamma_{2}$ and $\Gamma_{1}=1, \gamma=1$. Note that it is monotonically increasing with $f_{T}$ and reproduces above asymptotic limits.

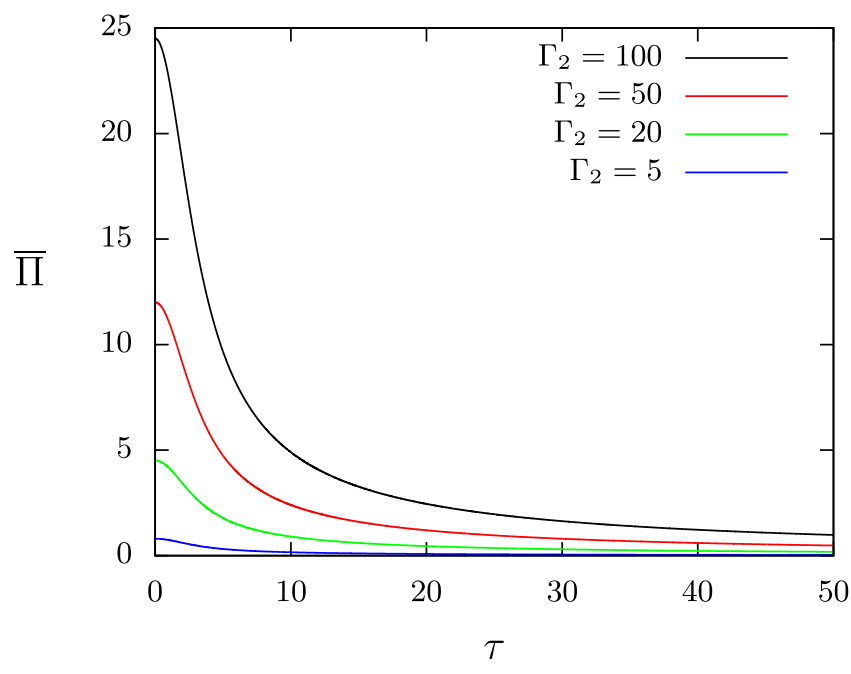

FIG. 1. Mean entropy production $\bar{\Pi}$ versus $\tau$ for distinct temperature sets $\Gamma_{1}=1$ and $\Gamma_{2}$ and $\gamma=1$. 


\section{FORCED BROWNIAN AND SEQUENTIAL RESERVOIRS}

Next, we extend analysis for the case of a Brownian particle in contact with sequential reservoirs and external forces. We shall focus on the two stage case and two simplest external forces protocols: constant and linear drivings. More specifically, the former is given by

$$
f_{i}(t)= \begin{cases}f_{1} ; & 0 \leqslant t<\tau / 2 \\ f_{2} ; & \tau / 2 \leqslant t<\tau\end{cases}
$$

where $f_{1}$ and $f_{2}$ denote their strengths in the first and second half period, respectively, whereas the latter case accounts for forces evolving linearly over the time according to the slopes:

$$
\frac{f_{i}(t)}{\gamma}=\left\{\begin{array}{c}
\lambda_{1} t ; \quad 0 \leqslant t<\tau / 2, \\
\lambda_{2}\left(\frac{\tau}{2}-t\right), \quad \tau / 2 \leqslant t<\tau,
\end{array}\right.
$$

with $\lambda_{1}$ and $\lambda_{2}$ being their amplitudes. It has been considered in Ref. [41] to compare the performance of distinct bounds between currents and the entropy production (TURs). In the presence of external forces, FP equation has the same form of Eq. (14), but now $\left\langle v_{i}\right\rangle(t)$ 's will be different from zero.

\section{A. Constant external forces}

From Eq. (13), the expressions for $\left\langle v_{i}\right\rangle(t)$ 's are given by

$$
\langle v\rangle=\left\{\begin{array}{c}
\left\langle v_{1}\right\rangle(t)=\frac{e^{\gamma \tau / 2}}{\gamma}\left(\frac{f_{2}-f_{1}}{1+e^{\gamma \tau / 2}}\right) e^{-\gamma t}+\frac{f_{1}}{\gamma}, \\
\left\langle v_{2}\right\rangle(t)=\frac{e^{\gamma \tau / 2}}{\gamma}\left(\frac{f_{1}-f_{2}}{1+e^{\gamma \tau / 2}}\right) e^{-\gamma(t-\tau / 2)}+\frac{f_{2}}{\gamma},
\end{array}\right.
$$

for the first or second half of each period, respectively.

The average work and heat per time are given by $\overline{\dot{W}}=$ $\bar{W}_{1}+\bar{W}_{2}$ and $\overline{\dot{Q}}=\overline{\dot{Q}}_{1}+\overline{\dot{Q}}_{2}$, respectively, and straightforwardly evaluated from Eq. (7), whose $\bar{W}_{1}$ and $\bar{Q}_{1}$ read

$$
\begin{aligned}
\overline{\dot{W}}_{1} & =-\frac{m f_{1}}{\tau} \int_{0}^{\tau / 2}\left\langle v_{1}\right\rangle d t \\
& =\frac{m f_{1}}{\gamma^{2} \tau}\left(f_{1}-f_{2}\right) \tanh \left(\frac{\gamma \tau}{4}\right)-\frac{m f_{1}^{2}}{2 \gamma}
\end{aligned}
$$

and

$$
\begin{aligned}
\bar{Q}_{1}= & \frac{m}{4 \gamma \tau}\left(\Gamma_{2}-\Gamma_{1}\right) \tanh \left(\frac{\gamma \tau}{2}\right)+\frac{m}{2 \gamma^{2} \tau}\left(f_{1}+f_{2}\right)^{2} \\
& \times \tanh \left(\frac{\gamma \tau}{4}\right)+\frac{2 m f_{1}^{2}}{\gamma^{2} \tau}\left[\frac{\gamma \tau}{4}-\tanh \left(\frac{\gamma \tau}{4}\right)\right],
\end{aligned}
$$

respectively. Analogous expressions are obtained for $\overline{\dot{W}}_{2}$ and $\overline{\dot{Q}}_{2}$ just by exchanging $1 \leftrightarrow 2$. Note that $\bar{Q}_{1}+\bar{Q}_{2}+\bar{W}_{1}+$ $\overline{\mathrm{W}}_{2}=0$, in consistency with the first law of thermodynamics.

In the same way as before, the steady entropy production per period $\bar{\Pi}$ can be evaluated from Eq. (10) (by taking $k_{\mathrm{B}}=$ 1) and reads

$$
\bar{\Pi}=\frac{2 \gamma}{m}\left(\frac{\overline{\dot{Q}}_{1}}{\Gamma_{1}}+\frac{\overline{\dot{Q}}_{2}}{\Gamma_{2}}\right)
$$

and we arrive at the following expression

$$
\begin{aligned}
\bar{\Pi}= & \frac{1}{2 \tau} \frac{\left(\Gamma_{2}-\Gamma_{1}\right)^{2}}{\Gamma_{1} \Gamma_{2}} \tanh \left(\frac{\gamma \tau}{2}\right)+\frac{1}{\gamma \tau}\left(\frac{1}{\Gamma_{1}}+\frac{1}{\Gamma_{2}}\right) \\
& \times \tanh \left(\frac{\gamma \tau}{4}\right)\left(f_{1}+f_{2}\right)^{2} \\
& +\left(\frac{f_{1}^{2}}{\Gamma_{1}}+\frac{f_{2}^{2}}{\Gamma_{2}}\right)\left[1-\frac{4}{\gamma \tau} \tanh \left(\frac{\gamma \tau}{4}\right)\right] .
\end{aligned}
$$

Since $\gamma \tau \geqslant 0$ and $1-\tanh (x) / x \geqslant 0$, it follows that $\bar{\Pi} \geqslant 0$. Note that $\bar{\Pi}$ reduces to Eq. (27) as $f_{1}=f_{2}=0$.

\section{Bilinear form and Onsager coefficients}

The shape of Eq. (36) is similar to the linear irreversible thermodynamics $[18,19,43]$, in which the entropy production is written down as a sum of flux-times-force expression. This similarity provides to reinterpret Eq. (36) in the following form:

$$
\bar{\Pi}=\mathcal{J}_{T} f_{T}+\mathcal{J}_{1} f_{1}+\mathcal{J}_{2} f_{2},
$$

where forces $f_{T}=\left(1 / \Gamma_{1}-1 / \Gamma_{2}\right)$ and $f_{1(2)}$ have associated fluxes $\mathcal{J}_{T}, \mathcal{J}_{1(2)}$ given by $\mathcal{J}_{T}=L_{T T} f_{T}$ [identical to Eq. (29)],

$$
\mathcal{J}_{1}=L_{11} f_{1}+L_{12} f_{2} \quad \text { and } \quad \mathcal{J}_{2}=L_{21} f_{1}+L_{22} f_{2},
$$

respectively, where $L_{11}, L_{12}, L_{21}$, and $L_{22}$ denote their Onsager coefficients given by

$$
L_{11}=\frac{1}{\Gamma_{1}}\left[1-\frac{3}{\gamma \tau} \tanh \left(\frac{\gamma \tau}{4}\right)\right]+\frac{1}{\gamma \tau \Gamma_{2}} \tanh \left(\frac{\gamma \tau}{4}\right)
$$

and

$$
L_{12}=L_{21}=\frac{1}{\gamma \tau}\left(\frac{1}{\Gamma_{1}}+\frac{1}{\Gamma_{2}}\right) \tanh \left(\frac{\gamma \tau}{4}\right),
$$

respectively. Coefficients $L_{22}$ and $L_{21}$ have the same shape of $L_{11}$ and $L_{12}$ by replacing $1 \leftrightarrow 2$, respectively. Besides, $L_{11}$ and $L_{22} \geqslant 0$ (as should be) and they satisfy the inequality $4 L_{11} L_{22}-\left(L_{12}+L_{21}\right)^{2} \geqslant 0$, in consistency with the positivity of the entropy production.

\section{B. Time-dependent external forces}

By repeating the previous calculations for linear external forces the mean velocities $\left\langle v_{i}\right\rangle(t)$ 's are given by

$$
\langle v\rangle=\left\{\begin{array}{l}
\left\langle v_{1}\right\rangle(t)=\frac{1}{\gamma}\left\{\lambda_{1}(\gamma t-1)\right. \\
\left.+e^{-\gamma t}\left[\lambda_{1}+\left(\lambda_{2} e^{\frac{\gamma \tau}{2}}-\lambda_{1}\right) \alpha(\gamma, \tau)\right]\right\}, \\
\left\langle v_{2}\right\rangle(t)=\frac{1}{\gamma}\left\{-\lambda_{2}\left[\gamma\left(t-\frac{\tau}{2}\right)-1\right]\right. \\
\left.+e^{-\gamma\left(t-\frac{\tau}{2}\right)}\left[\left(\lambda_{1} e^{\frac{\gamma \tau}{2}}-\lambda_{2}\right) \alpha(\gamma, \tau)-\lambda_{2}\right]\right\},
\end{array}\right.
$$

where

$$
\alpha(\gamma, \tau)=\frac{2-e^{\frac{\gamma \tau}{2}}(\gamma \tau-2)}{2\left(e^{\gamma \tau}-1\right)}
$$

respectively. Although more complex than the previous case, the mean work and heat per time are evaluated analogously 

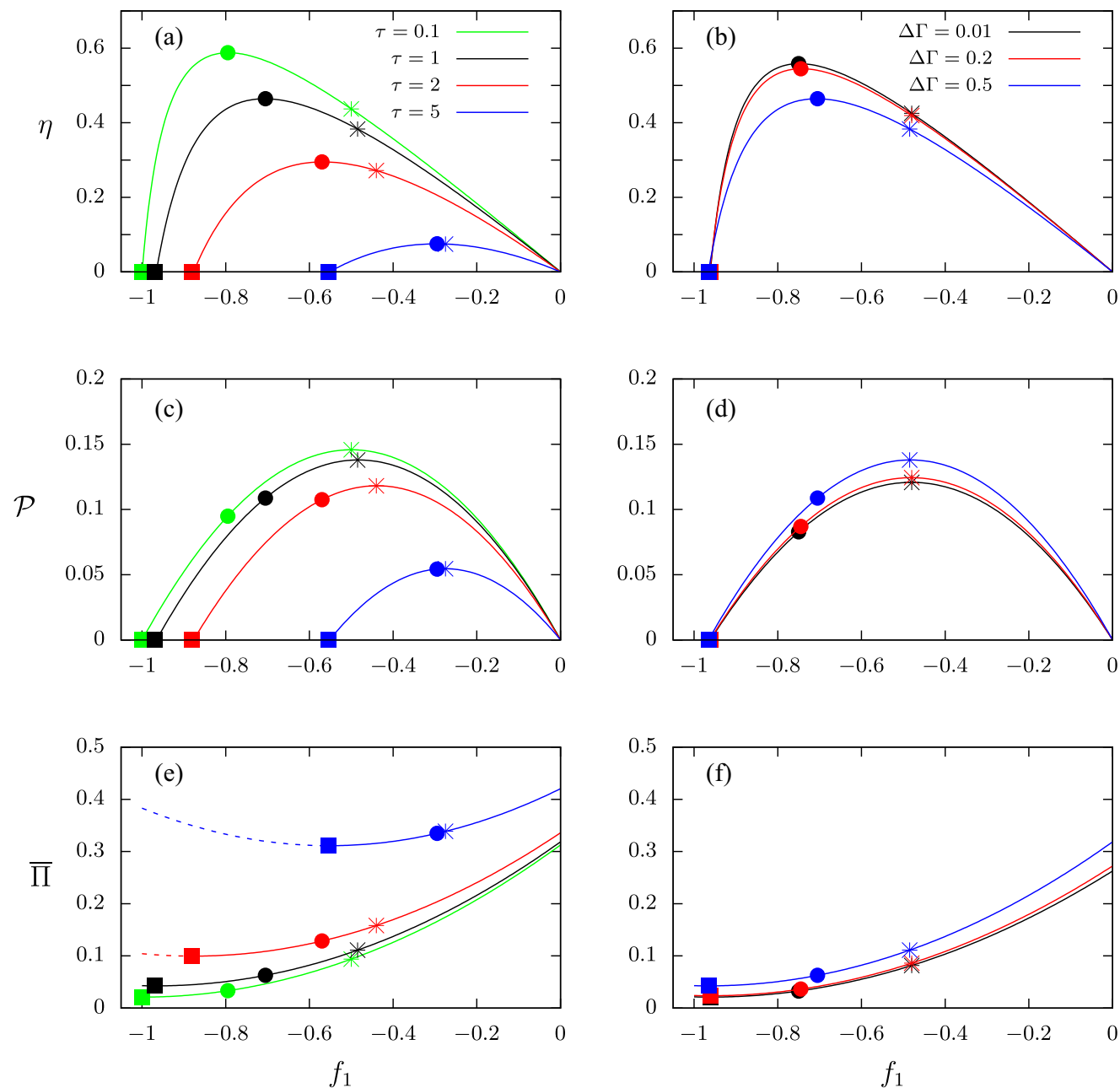

FIG. 2. Panels (a) and (b) depict the efficiency $\eta$ versus $f_{1}$ for distinct periods $\tau$ (for $\Delta \Gamma=0.5$ ) and $\Delta \Gamma$ 's (for $\tau=1$ ), respectively. In both cases, $\Gamma_{1}=2$ and $f_{2}=1$. Symbols $\bullet$, "stars," and "squares" denote the $f_{1 m E}, f_{1 m P}$, and $f_{1 m s}$, respectively. Panels (c) and (d) show the corresponding power $\mathcal{P}$, whereas (e) and (f) the average entropy production rate $\bar{\Pi}$. Dashed lines show the values of $f_{1}$ the system can not be operated as a thermal machine.

from expressions for $\left\langle v_{i}\right\rangle(t)$ 's and $b_{i}(t)$ 's, whose values averaged over a cycle read

$$
\begin{aligned}
\overline{\dot{W}}= & -\overline{\dot{Q}}=-\mathcal{A}\left\{e^{\gamma \tau} \varphi_{+}(\gamma, \tau, \xi)\right. \\
& \left.+12 e^{\frac{\gamma \tau}{2}}\left(\gamma^{2} \tau^{2} \xi-4\right)+\varphi_{-}(\gamma, \tau, \xi)\right\},
\end{aligned}
$$

where parameters $\mathcal{A}, \xi$ and $\varphi_{ \pm}(\gamma, \tau, \xi)$ read

$$
\mathcal{A}=\frac{m\left(\lambda_{1}+\lambda_{2}\right)^{2}}{24 \gamma^{2} \tau\left(e^{\gamma \tau}-1\right)}, \quad \xi=\frac{\lambda_{1} \lambda_{2}}{\left(\lambda_{1}+\lambda_{2}\right)^{2}},
$$

and

$$
\varphi_{ \pm}(\gamma, \tau, \xi)=\gamma^{2} \tau^{2}(2 \xi-1)(3 \pm \gamma \tau)+24(1 \pm \gamma \tau \xi),
$$

respectively.

\section{Bilinear form and Onsager coefficients}

As in the previous case, the entropy production has also the shape of Eqs. (37) and (38) given by $\bar{\Pi}=\mathcal{J}_{T} f_{T}+\mathcal{J}_{1} \lambda_{1}+$ $\mathcal{J}_{2} \lambda_{2}$, being $L_{T T}$ the same to Eq. (29), whereas the other
Onsager coefficients read

$$
\begin{aligned}
L_{11}= & \frac{1}{\Gamma_{1}}\left[\frac{\gamma^{2} \tau^{2}}{12}-\frac{\gamma \tau\left(2 e^{\gamma \tau}+1\right)}{4\left(e^{\gamma \tau}-1\right)}+\frac{1}{1+e^{-\frac{\gamma \tau}{2}}}\right. \\
& \left.+\frac{1}{\gamma \tau} \tanh \left(\frac{\gamma \tau}{4}\right)\right]+\frac{1}{\Gamma_{2}} \frac{\left[e^{\frac{\gamma \tau}{2}}(\gamma \tau-2)+2\right]^{2}}{4 \gamma \tau\left(e^{\gamma \tau}-1\right)},
\end{aligned}
$$

and

$$
L_{12}=\frac{\left(2 e^{\frac{\gamma \tau}{2}}-\gamma \tau-2\right)\left(2 e^{\frac{\gamma \tau}{2}}-\gamma \tau e^{\frac{\gamma \tau}{2}}-2\right)\left(\Gamma_{1}+\Gamma_{2}\right)}{4 \gamma \tau\left(e^{\gamma \tau}-1\right) \Gamma_{1} \Gamma_{2}},
$$

respectively. Coefficients $L_{22}$ and $L_{21}$ are again identical to $L_{11}$ and $L_{12}$ by exchanging $1 \leftrightarrow 2$. Also, it is straightforward to verify that $L_{11}$ and $L_{22}$ are strictly positive and $4 L_{11} L_{22}-$ $\left(L_{12}+L_{21}\right)^{2} \geqslant 0$.

\section{EFFICIENCY}

Distinct works have tackled the conditions in which periodically driven systems can operate as thermal machines 

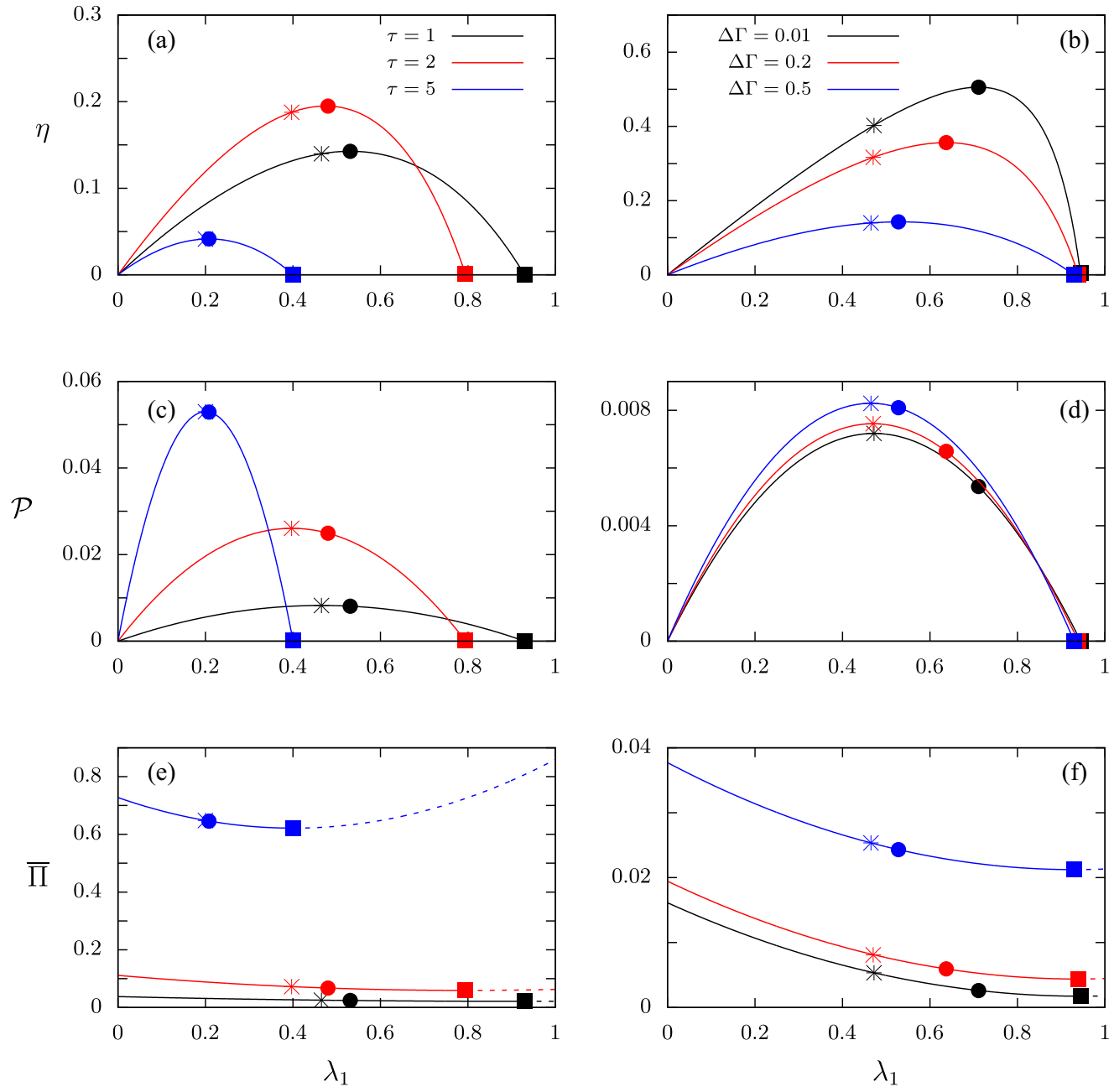

FIG. 3. Panels (a) and (b) depict the efficiency $\eta$ versus $\lambda_{1}$ for distinct periods $\tau$ (for $\Delta \Gamma=0.5$ ) and $\Delta \Gamma$ 's (for $\tau=1$ ), respectively. In both cases, $\Gamma_{1}=2$ and $\lambda_{2}=1$. Symbols $\bullet$, "stars," and "squares" denote the $\lambda_{1 m E}, \lambda_{1 m P}$, and $\lambda_{1 m s}$, respectively. Panels (c) and (d) show the corresponding power $\mathcal{P}$, whereas (e) and (f) the average entropy production rate $\bar{\Pi}$. Dashed lines show the values of $\lambda_{1}$ the system can not be operated as a thermal machine.

[18,43-47]. The conversion of a given type of energy into another one requires the existence of a generic force $X_{1}$ operating against its flux $J_{1} X_{1} \leqslant 0$ counterbalancing with driving forces $X_{2}$ and $X_{T}$ in which $J_{2} X_{2}+J_{T} X_{T} \geqslant 0$. A measure of efficiency $\eta$ is given by

$$
\begin{aligned}
\eta & =-\frac{\mathcal{J}_{1} X_{1}}{\mathcal{J}_{2} X_{2}+\mathcal{J}_{T} X_{T}} \\
& =-\frac{L_{11} X_{1}^{2}+L_{12} X_{1} X_{2}}{L_{21} X_{2} X_{1}+L_{22} X_{2}^{2}+L_{T T} X_{T}^{2}},
\end{aligned}
$$

where in such case $X_{T}=f_{T}$ and we have taken into account Eq. (37) for relating fluxes and Onsager coefficients. Taking into account that the best machine aims at maximizing the efficiency and minimizing the dissipation $\bar{\Pi}$ for a given power output $\mathcal{P}=-\Gamma_{1} \mathcal{J}_{1} X_{1}$, it is important to analyze the role of three load forces, $X_{1 m P}, X_{1 m E}$, and $X_{1 m S}$, in which the power output and efficiency are maximum and the dissipation is minimum, respectively [18]. Their values can be obtained straightforwardly from expressions for $\mathcal{P}$ and Eq. (45), respectively. Due to the present symmetric relation between Onsager coefficients $L_{12}=L_{21}$ (in both cases), they acquire simpler forms and read $2 X_{1 m P}=-L_{12} X_{2} / L_{11}$,

$$
X_{1 m E}=\frac{1}{L_{11} L_{12} X_{2}}\left[-L_{11}\left(L_{22} X_{2}^{2}+L_{T T} X_{T}^{2}\right)+A\left(X_{2}, X_{T}\right)\right],
$$

with $A\left(X_{2}, X_{T}\right)$ being given by

$$
\begin{aligned}
A\left(X_{2}, X_{T}\right)= & \sqrt{L_{11}\left(L_{22} X_{2}^{2}+L_{T T} X_{T}^{2}\right)} \\
& \times \sqrt{\left[L_{11}\left(L_{22} X_{2}^{2}+L_{T T} X_{T}^{2}\right)-L_{12}^{2} X_{2}^{2}\right]},
\end{aligned}
$$

and $X_{1 m S}=-L_{12} X_{2} / L_{11}=2 X_{1 m P}$, respectively, where $X_{i}=$ $f_{i}$ and $\lambda_{i}$ for the constant and linear drivings, respectively. The efficiencies at minimum dissipation, maximum power and its maximum value become $\eta_{m s}=0$,

$$
\eta_{m P}=\frac{L_{12}^{2} X_{2}^{2}}{2\left(2 L_{22} L_{11}-L_{12}^{2}\right) X_{2}^{2}+4 L_{T T} L_{11} X_{T T}^{2}},
$$


and

$\eta_{m E}=\frac{1}{L_{12}^{2} X_{2}^{2}}\left[2 L_{11}\left(L_{22} X_{2}^{2}+L_{T T} X_{T T}^{2}\right)-L_{12}^{2} X_{2}^{2}-2 A\left(X_{2}, X_{T}\right)\right]$,

respectively, and finally their associated power outputs read $\mathcal{P}_{m S}=0, \mathcal{P}_{m P}=\Gamma_{1} L_{12}^{2} X_{2}^{2} / 4 L_{11}$, and

$$
\begin{aligned}
\mathcal{P}_{m E}= & \frac{\Gamma_{1}}{L_{11} L_{12}^{2} X_{2}^{2}} \\
& \times\left[L_{11}\left(L_{22} X_{2}^{2}+L_{T T} X_{T}^{2}\right)-A\left(X_{2}, X_{T}\right)-L_{12}^{2} X_{2}^{2}\right] \\
& \times\left[L_{11}\left(L_{22} X_{2}^{2}+L_{T T} X_{T}^{2}\right)-A\left(X_{2}, X_{T}\right)\right]
\end{aligned}
$$

respectively. We pause to make a few comments: First, above expressions extend the findings from Ref. [18] for a couple of driving forces. Second, both efficiency and power vanish when $X_{1}=X_{1 m s}$ and $X_{1}=0$ and are strictly positive between those limits. Hence the physical regime in which the system can operate as an engine is bounded by the lowest entropy production $\bar{\Pi}_{m S}=L_{T T} X_{T}^{2}+\left(L_{22}-L_{12}^{2} / L_{11}\right) X_{2}^{2}$ and the value $\bar{\Pi}^{*}=L_{T T} X_{T}^{2}+L_{22} X_{2}^{2}$. Third, despite the long expressions for Eqs. (49) and (50), powers $\mathcal{P}_{m P}, \mathcal{P}_{m E}$ and efficiencies $\eta_{m P}, \eta_{m E}$ are linked through a couple of simple expressions (in similarity with Refs. $[18,46]$ ):

$$
\eta_{m P}=\frac{\eta_{m E}}{1+\eta_{m E}^{2}} \quad \text { and } \quad \frac{\mathcal{P}_{m E}}{\mathcal{P}_{m P}}=1-\eta_{m E}^{2},
$$

and they imply that $0 \leqslant \eta_{m P}<\eta_{m E}$ (with $0 \leqslant \eta_{m E} \leqslant 1$ and $0 \leqslant \eta_{m P} \leqslant 1 / 2$ ) and $0 \leqslant \mathcal{P}_{m E} \leqslant \mathcal{P}_{m P}$. Fourth and last, the achievement of most efficient machine $\eta_{m E}=1$ implies that the system has to be operated at null power $\mathcal{P}_{m E}=0$ and hence the projection of a machine operating for finite $\mathcal{P}_{m P} / \mathcal{P}_{m E}$ will imply at a loss of its efficiency.

Our purpose here aims at not only extending relevant concepts about efficiency for Brownian particles in contact with sequential reservoirs, but also to show that a desired compromise between maximum power and maximum efficiency can be achieved by adjusting conveniently the model parameters (such as the period and the driving). From expressions for Onsager coefficients, aforementioned quantities are evaluated, as depicted in Figs. 2 and 3 for distinct periods $\tau$ and temperature differences $\Delta \Gamma$ 's for constant and linear drivings, respectively. In both cases, quantities follow theoretical predictions and exhibit similar portraits, in which efficiencies and power outputs present maximum values at $f_{1 m E}\left(\lambda_{1 m E}\right)$ and $f_{1 m P}\left(\lambda_{1 m P}\right)$, respectively. The loss of efficiency from the maximum $\eta_{m E}$ as $f_{1}\left(\lambda_{1}\right)$ goes up (down) is signed by the increase of dissipation (as expected) until vanishing when $\bar{\Pi}=\bar{\Pi}^{*}$. For the constant driving, absolute values of forces and efficiencies increase as the period $\tau$ [see, e.g., panels (a)] and/or temperature differences [see, e.g., panels (b)] are lowered. In such a case, $\Gamma_{1} \approx \Gamma_{2}=\Gamma, \Delta \Gamma=$ $\Gamma_{1}-\Gamma_{2}<<1$ and the thermodynamic force $f_{T}$ approaches to $f_{T} \approx \Delta \Gamma / \Gamma^{2}$. Onsager coefficients become simpler in the limit of fast switchings, $\tau \rightarrow 0$ and $L_{11}, L_{22}, L_{12}$ approach to $\left(\Gamma_{1}+\Gamma_{2}\right) /\left(4 \Gamma_{1} \Gamma_{2}\right)$. Some remarkable quantities then approach to the asymptotic values $f_{1 m S} \rightarrow-f_{2}=2 f_{1 m P}$ and

$$
\eta_{m P} \rightarrow \frac{f_{2}^{2}\left(\Gamma_{1}+\Gamma_{2}\right)}{2\left[f_{2}^{2}\left(\Gamma_{1}+\Gamma_{2}\right)+2 \Delta \Gamma^{2}\right]},
$$

respectively. For $\Gamma_{1} \approx \Gamma_{2}, \eta_{m P} \rightarrow 1 / 2, \eta_{m E} \rightarrow 1$, and $\mathcal{P}_{m P}$ reads $\mathcal{P}_{m P} \rightarrow f_{2}^{2} / 8$ and thereby the limit of an ideal machine is achieved for low periods and equal temperatures. Similar features are verified for the linear driving, including increasing efficiencies as both $\tau$ and $\Delta \Gamma$ decreases. However, they are marked by a reentrant behavior for $\tau<<1$ and $\Delta \Gamma \neq 0$ [see, e.g., Figs. 3(a) and 5]. It moves for lower $\tau$ 's as $\Delta \Gamma$ goes down and the limit of ideal machine, $\eta_{m P} \rightarrow 1 / 2$ and $\eta_{m E} \rightarrow 1$, is also recovered when both $\tau \rightarrow 0$ for $\Delta \Gamma \rightarrow 0$.
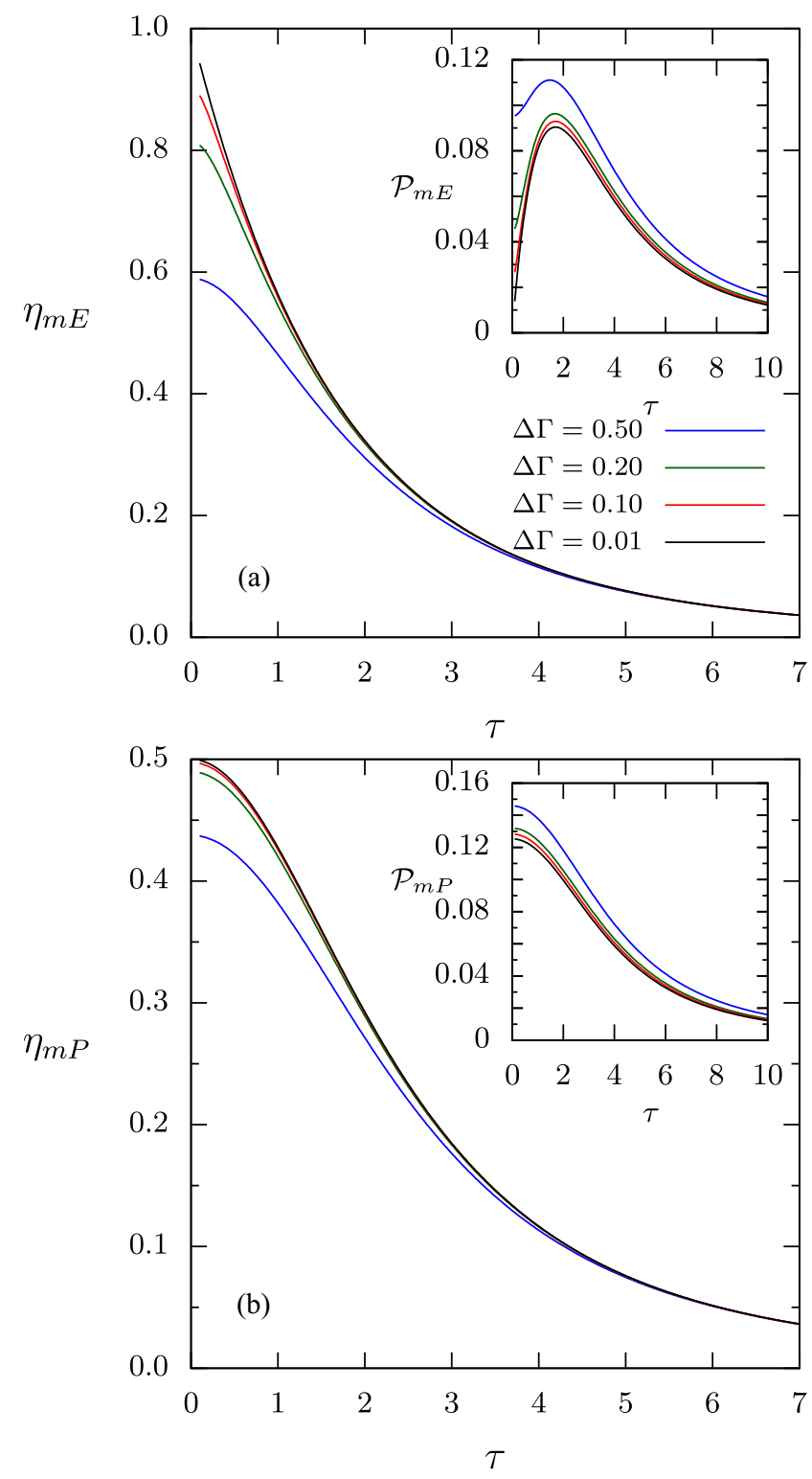

FIG. 4. For $\Gamma_{1}=2, f_{2}=1$ and distinct $\Delta \Gamma$ 's, the comparison between maximum efficiency [panel (a)] and efficiency at maximum power [panel (b)] for constant drivings. Insets: The corresponding power outputs $\mathcal{P}$ 's versus $\tau$. 

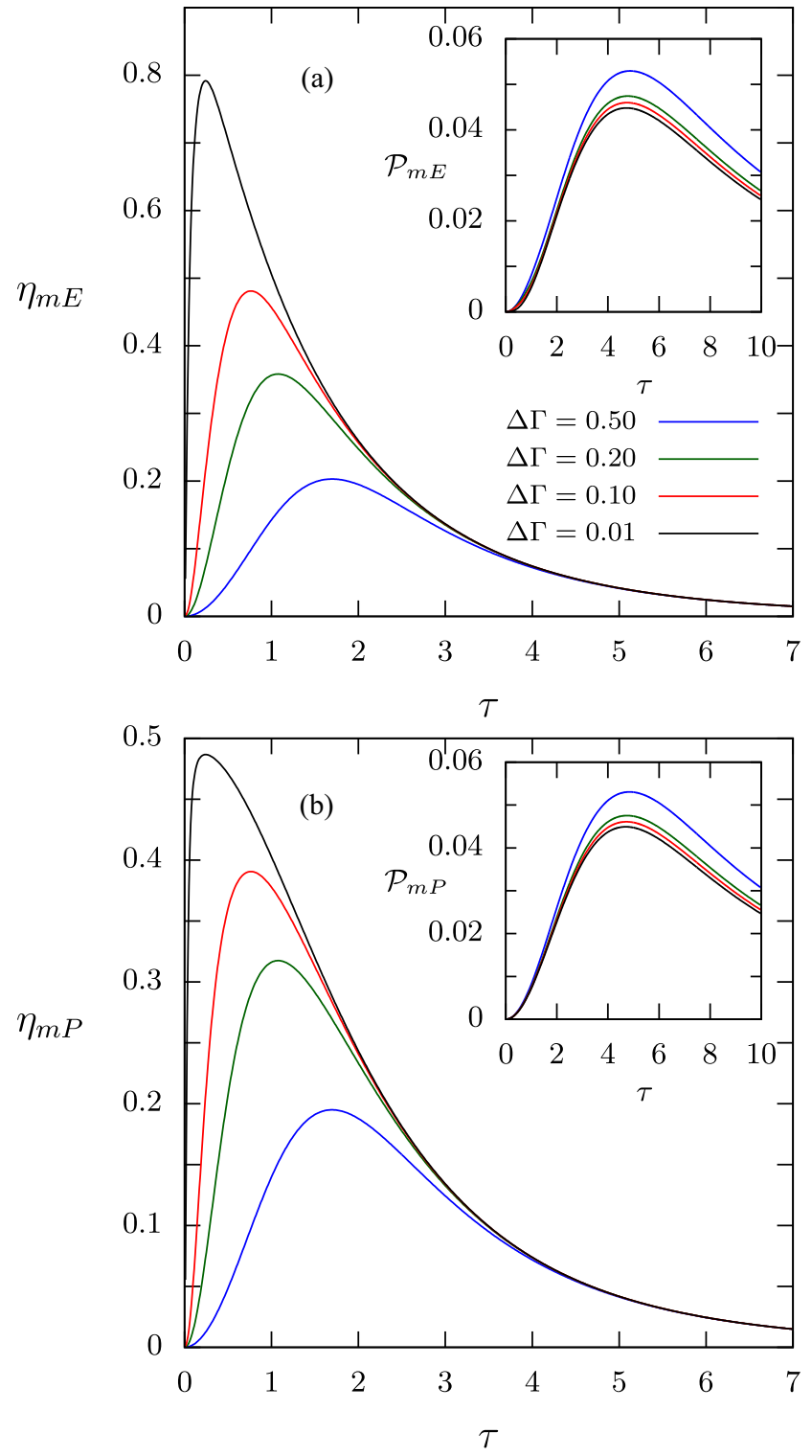

FIG. 5. For $\Gamma_{1}=2, \lambda_{2}=1$, and distinct $\Delta \Gamma$ 's, the comparison between maximum efficiency [panel (a)] and efficiency at maximum power [panel (b)] for linear drivings. Insets: The corresponding power outputs $\mathcal{P}$ 's versus $\tau$.
Other differences between protocols are appraised in Figs. 4 and 5. For finite difference of temperatures, the constant driving is always more efficient than the linear one and their power outputs are also superior. The maximum efficiency curves (linear drivings) are also reentrant, whose maxima values increase and deviate for lower $\tau$ 's as $\Delta \Gamma$ decreases.

We close this section by remarking that although short periods indicates a general route for optimizing the efficiency of thermal machines in contact to sequential reservoirs, the present description provides to properly tune the period and forces to obtain the desirable compromise between maximum efficiency and power.

\section{CONCLUSIONS}

The thermodynamics of a Brownian particle periodically placed in contact with sequential thermal reservoirs is introduced. We have obtained explicit (exact) expressions for relevant quantities, such as heat, work, and entropy production. Generalization for an arbitrary number of sequential reservoirs and the influence of external forces were considered. Considerations about the efficiency were undertaken, in which Brownian machines can be properly operated ensuring the reliable compromise between efficiency and power for small switching periods.

As a final comment, we mention the several perspectives to be addressed. First, it might be very interesting to extend such a study for other external forces protocols (e.g., sinusoidal time-dependent ones) as well as for time asymmetric switchings, to compare their efficiencies, mainly with the linear driving case. Finally, it would be remarkable to verify the validity of recent proposed uncertainties relations (TURs) for Fokker-Planck equations [39,41], in such a class of systems.

\section{ACKNOWLEDGMENTS}

We acknowledge Karel Proesmans and Mário J. de Oliveira for careful readings of the manuscript and useful suggestions. C.E.F. acknowledges the financial support from Fundação de Amparo à Pesquisa do estado de São Paulo (FAPESP) under Grant No. 2018/02405-1. Authors also acknowledge the financial support from Brazilian Agencies CAPES and CNPq.
[1] I. Prigogine, Introduction to Thermodynamics of Irreversible Processes (Interscience, New York, 1965).

[2] S. R. De Groot and P. Mazur, Non-Equilibrium Thermodynamics (North-Holland, Amsterdam, 1962).

[3] T. Tomé and M. J. De Oliveira, Stochastic Dynamics and Irreversibility (Springer, Berlin, 2015).

[4] U. Seifert, Rep. Prog. Phys. 75, 126001 (2012).

[5] C. Van den Broeck and M. Esposito, Physica A 418, 6 (2015).

[6] C. Jarzynski, Phys. Rev. Lett. 78, 2690 (1997).

[7] O.-P. Saira, Y. Yoon, T. Tanttu, M. Möttönen, D. V. Averin, and J. P. Pekola, Phys. Rev. Lett. 109, 180601 (2012).

[8] K. Proesmans and C. Van den Broeck, Europhys. Lett. 119, 20001 (2017).
[9] A. C. Barato and U. Seifert, Phys. Rev. Lett. 114, 158101 (2015).

[10] J. Schnakenberg, Rev. Mod. Phys. 48, 571 (1976).

[11] C. E. Fernández Noa, P. E. Harunari, M. J. de Oliveira, and C. E. Fiore, Phys. Rev. E 100, 012104 (2019).

[12] T. Herpich, J. Thingna, and M. Esposito, Phys. Rev. X 8, 031056 (2018).

[13] T. Herpich and M. Esposito, Phys. Rev. E 99, 022135 (2019).

[14] B. O. Goes, C. E. Fiore, and G. T. Landi, Phys. Rev. Res. 2, 013136 (2020).

[15] H. Ge, M. Qian, and H. Qian, Phys. Rep. 510, 87 (2012).

[16] T. Tomé and M. J. de Oliveira, Phys. Rev. E 91, 042140 (2015). 
[17] K. Brandner, K. Saito, and U. Seifert, Phys. Rev. X 5, 031019 (2015).

[18] K. Proesmans, B. Cleuren, and C. Van den Broeck, Phys. Rev. Lett. 116, 220601 (2016).

[19] K. Proesmans and C. E. Fiore, Phys. Rev. E 100, 022141 (2019).

[20] B. Cleuren and K. Proesmans, Physica A 552, 122789 (2020).

[21] M. Pezzutto, M. Paternostro, and Y. Omar, New J. Phys. 18, 123018 (2016).

[22] M. Pezzutto, M. Paternostro, and Y. Omar, Quant. Sci. Technol. 4, 025002 (2019).

[23] G. T. Landi, E. Novais, M. J. de Oliveira, and D. Karevski, Phys. Rev. E 90, 042142 (2014).

[24] C. H. Bennett, Int. J. Theor. Phys. 21, 905 (1982).

[25] K. Maruyama, F. Nori, and V. Vedral, Rev. Mod. Phys. 81, 1 (2009).

[26] T. Sagawa, J. Stat. Mech.: Theory Exp. (2014) P03025.

[27] J. M. Parrondo, J. M. Horowitz, and T. Sagawa, Nat. Phys. 11, 131 (2015).

[28] V. Giovannetti and G. M. Palma, Phys. Rev. Lett. 108, 040401 (2012).

[29] P. Strasberg, G. Schaller, T. Brandes, and M. Esposito, Phys. Rev. X 7, 021003 (2017).

[30] F. L. S. Rodrigues, G. De Chiara, M. Paternostro, and G. T. Landi, Phys. Rev. Lett. 123, 140601 (2019).

[31] C. Van den Broeck, Phys. Rev. Lett. 95, 190602 (2005).
[32] T. Schmiedl and U. Seifert, Europhys. Lett. 83, 30005 (2008).

[33] F. Berger, T. Schmiedl, and U. Seifert, Phys. Rev. E 79, 031118 (2009).

[34] A. Dechant, N. Kiesel, and E. Lutz, Europhys. Lett. 119, 50003 (2017).

[35] Y. Izumida and K. Okuda, Eur. Phys. J. B 77, 499 (2010).

[36] D. Abreu and U. Seifert, Europhys. Lett. 94, 10001 (2011).

[37] A. C. Barato and U. Seifert, J. Phys. Chem. B 119, 6555 (2015).

[38] T. R. Gingrich, J. M. Horowitz, N. Perunov, and J. L. England, Phys. Rev. Lett. 116, 120601 (2016).

[39] Y. Hasegawa and T. Van Vu, Phys. Rev. E 99, 062126 (2019).

[40] T. Van Vu and Y. Hasegawa, Phys. Rev. E 100, 012134 (2019).

[41] T. Van Vu and Y. Hasegawa, Phys. Rev. Res. 2, 013060 (2020).

[42] T. Tomé and M. J. de Oliveira, Phys. Rev. E 82, 021120 (2010).

[43] K. Proesmans and C. Van den Broeck, Phys. Rev. Lett. 115, 090601 (2015).

[44] M. Bauer, K. Brandner, and U. Seifert, Phys. Rev. E 93, 042112 (2016).

[45] A. Rosas, C. Van den Broeck, and K. Lindenberg, Phys. Rev. E 94, 052129 (2016).

[46] K. Proesmans and C. Van den Broeck, Chaos: Interdiscip. J Nonlin. Sci. 27, 104601 (2017).

[47] A. Rosas, C. Van den Broeck, and K. Lindenberg, Phys. Rev. E 96, 052135 (2017). 


\section{Bibliografia}

[1] Georgy Lebon, David Jou e José Casas-Vázquez. Understanding non-equilibrium thermodynamics. Vol. 295. Springer, 2008.

[2] Per Chr Hemmer, Helge Holden e Signe Kjelstrup. Collected Works Of Lars Onsager, The (With Commentary). Vol. 17. World Scientific, 1996.

[3] Gatien Verley et al. "The unlikely Carnot efficiency". Em: Nature communications 5.1 (2014), pp. 1-5.

[4] Michael Bauer, Kay Brandner e Udo Seifert. "Optimal performance of periodically driven, stochastic heat engines under limited control”. Em: Physical Review E 93.4 (2016), p. 042112.

[5] Tim Schmiedl e Udo Seifert. "Efficiency at maximum power: An analytically solvable model for stochastic heat engines". Em: Europhysics Letters 81.2 (2007), p. 20003.

[6] Karel Proesmans e Christian Van den Broeck. "The underdamped Brownian duet and stochastic linear irreversible thermodynamics". Em: Chaos: An Interdisciplinary Journal of Nonlinear Science 27.10 (2017), p. 104601.

[7] Ignacio A Martınez et al. "Brownian carnot engine". Em: Nature physics 12.1 (2016), pp. 67-70. 
[8] Karel Proesmans et al. "Brownian Duet: A Novel Tale of Thermodynamic Efficiency". Em: Phys. Rev. X 6 (4 out. de 2016), p. 041010. DOI: 10 . 1103 / PhysRevX.6.041010. URL: https://link.aps.org/doi/10.1103/ PhysRevX.6.041010.

[9] Sudeesh Krishnamurthy et al. "A micrometre-sized heat engine operating between bacterial reservoirs". Em: Nature Physics 12.12 (2016), pp. 1134-1138.

[10] Valentin Blickle e Clemens Bechinger. "Realization of a micrometre-sized stochastic heat engine". Em: Nature Physics 8.2 (2012), pp. 143-146.

[11] Marie Chupeau et al. "Engineered swift equilibration for Brownian objects: from underdamped to overdamped dynamics”. Em: New Journal of Physics 20.7 (jul. de 2018), p. 075003. DOI: 10 . 1088/1367-2630/aac875. URL: https : / / doi.org/10.1088/1367-2630/aac875.

[12] Frank L Curzon e B Ahlborn. "Efficiency of a Carnot engine at maximum power output”. Em: American Journal of Physics 43.1 (1975), pp. 22-24.

[13] II Novikov. "The efficiency of atomic power stations (a review)". Em: Journal of Nuclear Energy (1954) 7.1-2 (1958), pp. 125-128.

[14] Christian Van den Broeck. "Thermodynamic efficiency at maximum power". Em: Physical review letters 95.19 (2005), p. 190602.

[15] CE Fernández Noa, William GC Oropesa e CE Fiore. "Thermodynamics of collisional models for Brownian particles: General properties and efficiency”. Em: Physical Review Research 2.4 (2020), p. 043016.

[16] Don S Lemons. An Introduction to Stochastic Processes in Physics. 2003.

[17] Karel Proesmans e Christian Van den Broeck. "Onsager coefficients in periodically driven systems”. Em: Physical review letters 115.9 (2015), p. 090601. URL: https://journals.aps.org/prl/abstract/10.1103/PhysRevLett. 115.090601 .

[18] Michael Bauer, Kay Brandner e Udo Seifert. "Optimal performance of periodically driven, stochastic heat engines under limited control". Em: Physical Review E 93.4 (2016), p. 042112. URL: https: / / journals.aps.org/pre/abstract/ $10.1103 /$ PhysRevE.93.042112. 
[19] Alexandre Rosas, Christian Van den Broeck e Katja Lindenberg. "Onsager coefficients for systems with periodic potentials”. Em: Physical Review E 94.5 (2016), p. 052129. URL: https:// journals. aps. org/pre/abstract/10 . 1103 /PhysRevE.94.052129.

[20] Karel Proesmans e Christian Van den Broeck. "The underdamped Brownian duet and stochastic linear irreversible thermodynamics". Em: Chaos: An Interdisciplinary Journal of Nonlinear Science 27.10 (2017), p. 104601. URL: https : / / aip.scitation.org/doi/abs/10.1063/1.5001187.

[21] Karel Proesmans, Bart Cleuren e Christian Van den Broeck. "Power-efficiencydissipation relations in linear thermodynamics". Em: Physical review letters 116.22 (2016), p. 220601.

[22] Alexandre Rosas, Christian Van den Broeck e Katja Lindenberg. "Stochastic thermodynamics for a periodically driven single-particle pump". Em: Physical Review E 96.5 (2017), p. 052135. URL: https : / / journals . aps . org / pre / abstract/10.1103/PhysRevE.96.052135.

[23] Yoshihiko Hasegawa e Tan Van Vu. "Uncertainty relations in stochastic processes: An information inequality approach”. Em: Physical Review E 99.6 (2019), p. 062126. URL: https: / / journals. aps . org/pre/abstract/ 10 . 1103 /PhysRevE.99.062126.

[24] Tan Van Vu e Yoshihiko Hasegawa. "Thermodynamic uncertainty relations under arbitrary control protocols". Em: Phys. Rev. Research 2 (1 jan. de 2020), p. 013060. DOI: 10.1103 / PhysRevResearch.2.013060. URL: https : / / ink . aps.org/doi/10.1103/PhysRevResearch.2.013060. 